\title{
A Grid based Indoor Radiolocation Technique Based on Spatially Coherent Path Loss Model
}

\author{
Murat Ambarkutuk \\ Thesis submitted to the Faculty of the \\ Virginia Polytechnic Institute and State University \\ in partial fulfillment of the requirements for the degree of \\ Master of Science \\ in \\ Mechanical Engineering
}

Tomonari Furukawa, Chair

Pinhas Ben-Tzvi

Alan T. Asbeck

September 1, 2017

Blacksburg, Virginia

Keywords: Indoor localization, Radiolocation, Path Loss Modeling

Copyright 2017, Murat Ambarkutuk 


\section{A Grid based Indoor Radiolocation Technique Based on Spatially Coherent Path Loss Model}

Murat Ambarkutuk

ABSTRACT (Academic)

This thesis presents a grid-based indoor radiolocation technique based on a Spatially Coherent Path Loss Model (SCPL). SCPL is a path loss model which characterizes the radio wave propagation in an environment by solely using Received Signal Strength (RSS) fingerprints. The propagation of the radio waves is characterized by uniformly dividing the environment into grid cells, followed by the estimation of the propagation parameters for each grid cell individually. By using SCPL and RSS fingerprints acquired at an unknown location, the distance between an agent and all the access point in an indoor environment can be determined. A least-squares based trilateration is then used as the global fix of location the agent in the environment. The result of the trilateration is then represented in a probability distribution function over the grid cells induced by SCPL. Since the proposed technique is able to locally model the propagation accounting for attenuation of non-uniform environmental irregularities, the characterization of the path loss in the indoor environment and radiolocation technique might yield improved results. The efficacy of the proposed technique was investigated with an experiment comparing SCPL and an indoor radiolocation technique based on a conventional path loss model. 


\title{
A Grid based Indoor Radiolocation Technique Based on Spatially Coherent Path Loss Model
}

Murat Ambarkutuk

\author{
ABSTRACT (General Audience)
}

This thesis presents a technique uses radio waves to localize an agent in an indoor environment. By characterizing the difference between transmitted and received power of the radio waves, the agent can determine how far it is away from the transmitting antennas, i.e. access points, placed in the environment. Since the power difference mainly results from obstructions in the environment, the attenuation profile of the environment carries a significant importance in radiolocation techniques. The proposed technique, called Spatially Coherent Path Loss Model (SCPL), characterizes the radio wave propagation, i.e. the attenuation, separately for different regions of the environment, unlike the conventional techniques employing global attenuation profiles. The localization environment is represented with grid-cell structure and the parameters of SCPL model describing the extent of the attenuation of the environment are estimated individually. After creating a attenuation profile of the environment, the agent localizes itself in the localization environment by using SCPL with signal powers received from the access points. This scheme of attenuation profiling constitutes the main contribution of the proposed technique. The efficacy and validity of the proposed technique was investigated with an experiment comparing SCPL and an indoor radiolocation technique based on a conventional path loss model. 


\section{Dedication}

To my loving parents, lovely sister, and my precious friend Isil Anakok. 


\section{Acknowledgments}

To begin, I would like to thank Ministry of National Education of Turkish Government for providing me this opportunity with the scholarship I was granted. Without this scholarship, I could not have the chance the expand my horizon socially and academically.

I also would like to thank Professor Tomonari Furukawa for his continuous support and guidance during my study. Not only I honed my academic skills as a researcher, but did I also furthered my competence in scholarly world with his constant feedback.

I would like to give praise to the members, Kuya, Boren, George, Josiah, Song, Luan, Yoon, Chris, Affan, Rich, Hangxin, Tian, Yazhe, Orson, Urvi, Jihong, Diya, Peter, and Cameron, in Computational Multi-physics Laboratory in Virginia Tech for their friendship, and support. Through your help and input, I was able to navigate in the graduate life better.

I would like to express my gratitude to Srivatsan and Savio for your cooperation, and endless support.

I would like to thank my family who encouraged and supported me even from a great distance. Also I would like to express my gratitude for Fedora family who has accepted me as a part of their family. I would not be in this position, if it had not been their encouragement.

Lastly, I would like to thank Virginia Tech for the welcoming and supporting environment provided for me, which enabled me to keep my focus on my research. 


\section{Contents}

List of Figures $\quad$ x

List of Tables $\quad$ xiii

1 Introduction $\quad 1$

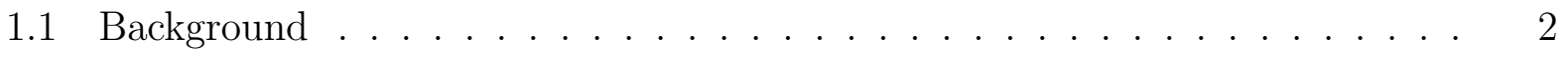

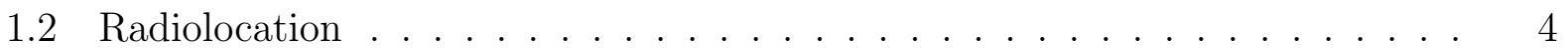

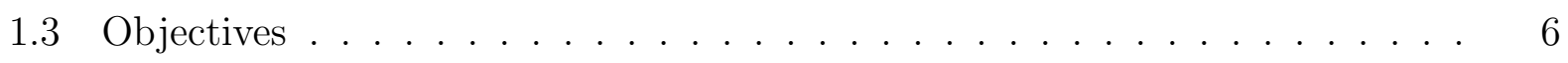

1.4 A Grid-based Indoor Localization Technique based on Spatially Coherent Path Loss Model . . . . . . . . . . . . . . . . . . . . 6

1.5 Summary of Contributions . . . . . . . . . . . . . . . . . 7

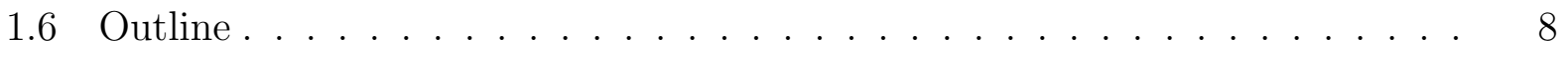

1.7 Summary . . . . . . . . . . . . . . . . . . . . 9

2 Literature Review $\quad 10$

2.1 Radiolocation Techniques based on Time-based Measurements . . . . . . . . 11 
2.2 Radiolocation Techniques based on Power-based Measurements . . . . . . . 15

2.2.1 Data-driven Approaches .................... 15

2.2.2 Model-based Approaches ................... 18

2.3 Radiolocation Techniques based on Angle of Arrival Measurements . . . . . . 20

2.4 Radiolocation Techniques based on Channel Characteristics . . . . . . . . . 22

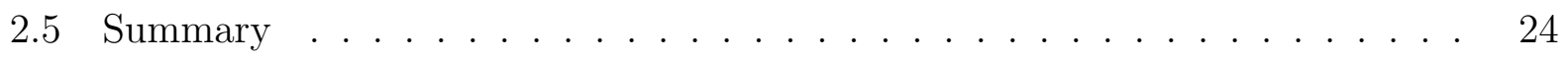

3 Radio Wave Propagation in Indoor Radiolocation 26

3.1 Radio Wave Propagation . . . . . . . . . . . . . . . . 26

3.2 Radio Wave Propagation Mechanisms . . . . . . . . . . . . . . . 28

3.2.1 Free Path Propagation . . . . . . . . . . . . . . 29

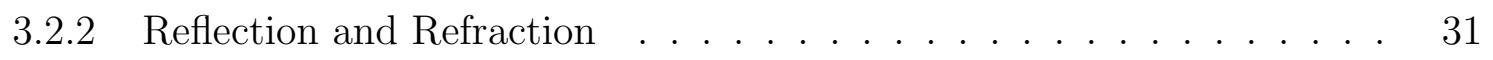

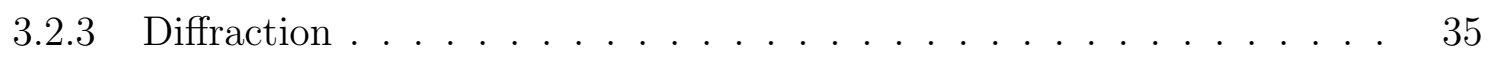

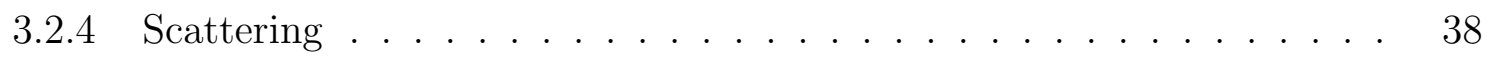

3.3 Log-distance Path Loss Model . . . . . . . . . . . . . . . . . . . 39

3.4 Indoor Radiolocation with Trilateration . . . . . . . . . . . . . . 41

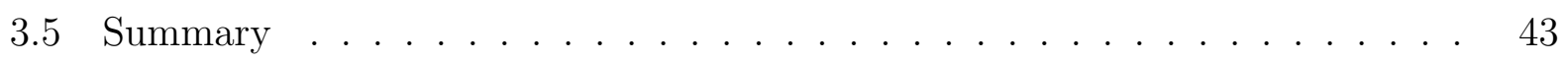

4 Grid Based Indoor Radiolocation Technique Based on SCPL 45

4.1 Spatially Coherent Path Loss Model . . . . . . . . . . . . . . . . . . . 47

4.1 .1 Model Parameters .................... 48 
4.1.2 Parameter Estimation for Spatially Coherent Path Loss Model (SCPL) Model . . . . . . . . . . . . . . . . . . . . . . 52

4.2 Grid-based Radiolocation Based on SCPL Model . . . . . . . . . . . . . 54

4.2.1 Parameter Learning and Parameter Selection . . . . . . . . . . . 54

4.2.2 Separation Distance Estimation and Target Localization $\ldots \ldots . . .56$

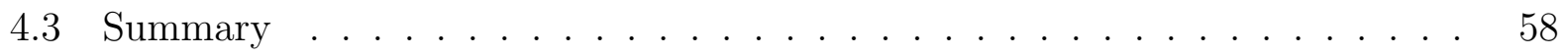

5 Experiments and Results $\quad 60$

$5.1 \quad$ Experimental Setup . . . . . . . . . . . . . . . . . . . . 60 60

$5.1 .1 \quad$ Localization Environment . . . . . . . . . . . . . . . 60

5.1 .2 Structure of the Environment . . . . . . . . . . . . . . 61

5.1 .3 Radio Modules . . . . . . . . . . . . . . . . . . . . . . 63

5.1 .4 Dataset . . . . . . . . . . . . . . . . . . . 63

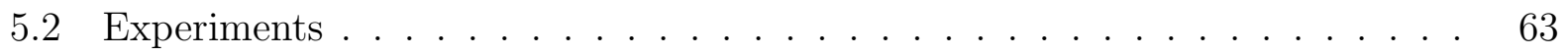

5.2 .1 Path Loss Estimation with SCPL . . . . . . . . . . . . . 63

5.2 .2 Analysis of SCPL Model Parameters . . . . . . . . . . . . . . 67

5.2 .3 Analysis of Estimated Separation Distance . . . . . . . . . . . . 68

5.2 .4 Analysis of Target Localization _ . . . . . . . . . . . . 70

5.2 .5 Access Point Analysis _ . . . . . . . . . . . . . . . . 72

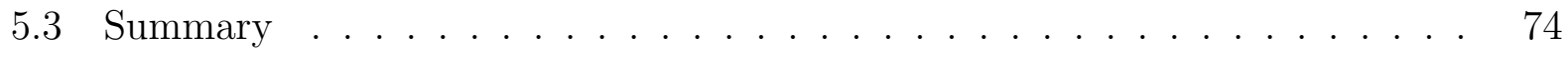


6 Conclusions and Future Work

$\begin{array}{ll}\text { Bibliography } & 78\end{array}$

Appendix A 2017 IEEE International Conference on Multisensor Fusion and Integration for Intelligent Systems. 


\section{List of Figures}

1.1 Line-of-sight (LOS) Coverage of a generic environment $\ldots \ldots . \ldots$

1.2 A typical floor plan with LOS and NLOS coverage . . . . . . . . . . . 5

2.1 Radiolocation Techniques based on Measurements Employed . . . . . . . . . 11

2.2 Localization of the target with time of arrival fingerprints . . . . . . . . . 12

2.3 Localization of the target with time difference of arrival fingerprints . . . . 13

2.4 Data-driven Fingerprinting-based Radiolocation Approach . . . . . . . . . 16

2.5 Model-based Fingerprinting Radiolocation Approach _. . . . . . . . . . . 19

2.6 Angle-of-Arrival Determination with Multiple Antennas . . . . . . . . . . . . 21

3.1 Friis' Free-space Propagation Model . . . . . . . . . . . . . . . . . . . . . . . 30

3.2 Expected Free Space Path Loss as a Function of Distance . . . . . . . . . . . 31

3.3 Reflection and Refraction of a Radio Wave . . . . . . . . . . . . . . 32

3.4 Two-ray Reflection Model . . . . . . . . . . . . . . . . . . . . . . . . 33 
3.5 Comparison of the received signal levels of Two-ray Reflection Model and Free-space Propagation Model . . . . . . . . . . . . . . . . . . . . . . . 35

3.6 Diffraction mechanism in an indoor environment . . . . . . . . . . . . 36

3.7 Fresnel Knife Edge Diffraction Model Parameters . . . . . . . . . . . . . . . 37

3.8 Scattering Mechanism . . . . . . . . . . . . . . . . . . . . 38

3.9 LDPL Model Parameters and Path Loss . . . . . . . . . . . . . . . . 40

3.10 Trilateration of an agent with separation distance estimations $\ldots . . . .44$

4.1 Indoor radiolocation problem in consideration . . . . . . . . . . . 46

4.2 Schematic diagram of the proposed localization algorithm . . . . . . . . 47

4.3 Localization Environment _ . . . . . . . . . . . . . . . . . . . . . . . . 48

4.4 Propagation characterization for each grid cell . . . . . . . . . . . . . . . 49

4.5 Path Loss Measurements with Increasing Path Loss Exponent $n_{g} \ldots \ldots$

4.6 Distance Correction Factor . . . . . . . . . . . . . . . . . . . . . . . . 51

4.7 Path Loss Measurements with Increasing Shadowing Factor $\sigma_{g} \ldots \ldots$

4.8 Parameter Estimation for SCPL Model . . . . . . . . . . . . . . . 53

4.9 Parameter Learning with a Feed-forward Neural Network and Parameter Selection with the Trained Network _. . . . . . . . . . . . 55

4.10 Separation Distance Estimation with SCPL $\ldots \ldots \ldots \ldots$

5.1 The position of obstructions in the environment . . . . . . . . 61

5.2 Far-field Region for Radio Waves . . . . . . . . . . . . . . . . 62 
5.3 Panaromic photograph of the experimentation site . . . . . . . . . . 63

5.4 Measured mean path loss of each grid cell at $900 \mathrm{MHz}$ frequency channel . . 64

5.5 Path Loss Estimation Error at $900 \mathrm{MHz}$ frequency channel . . . . . . . . . 65

5.6 Measured mean path loss of each grid cell at $2400 \mathrm{MHz}$ frequency channel . 66

5.7 Path Loss Estimation Error at $2400 \mathrm{MHz}$ frequency channel . . . . . . . . 67

5.8 Estimated propagation parameters vs. grid cells $\ldots \ldots \ldots$. . . . . . 68

5.9 Cumulative distribution function of range estimation errors . . . . . . . . . 69

5.10 Histogram of Separation Distance Error for LDPL and SCPL . . . . . . 70

5.11 Cumulative distribution function of target localization error. . . . . . . . 71

5.12 Error distribution of both algorithms over grid cells. . . . . . . . . . 72

5.13 Histogram of Target Localization Error for LDPL and SCPL . . . . . . . 72

6.1 Location-based Access Point Selection algorithm . . . . . . . . . . . . 77

6.2 State Estimation and Target Tracking . . . . . . . . . . . . . 77 


\section{List of Tables}

3.1 Model parameters of the Friis' Free-space Equation $\ldots$. . . . . . . . . . . . 29

3.2 Path loss exponent values in different environments . . . . . . . . . . 40

4.1 The descriptions of SCPL model parameters. . . . . . . . . . . . . . 49

5.1 Comparison of Errors in Separation Distance Estimation $\ldots . . . . . .70$

5.2 Comparison of Errors in Target Localization . . . . . . . . . . . . 71

5.3 Error Statistics of Access Points in Separation Distance Estimation . . . . 73

5.4 Comparison of Error Statistics in Separation Distance Estimation with Best 3 and Worst 3 access points . . . . . . . . . . . . . . . . 74 


\section{Nomenclature}

AoA Angle of Arrival. 20, 21, 24

FFSE Friis' Free Space Equation. 29, 31, 33, 34, 47

LDPL Log-distance Path Loss. 5, 6, 18-20, 39, 40, 50, 52, 62, 64-71, 73-76

LOS Line-of-sight. x, 2-5, 7, 8, 12, 13, 15, 21, 24, 29, 31-33, 35, 37, 40, 47, 49, 51, 61

MIMO Multiple-Input Multiple-Output. 20, 22

N-LOS Non-Line-of-sight. 2-5, 7, 8, 12-14, 23, 24, 35, 38, 40, 43, 49-51, 67, 68, 76

RSS Received Signal Strength. ii, 5-7, 15-19, 39, 41, 45, 46, 52, 56

SAR Synthetic-aperture Radar. 22

SCPL Spatially Coherent Path Loss Model. ii, iii, viii, xi, xiii, 6-8, 45-49, 52-56, 58, 60, $62,64-71,73-76$

TDoA Time Difference of Arrival. 11-13

ToA Time of Arrival. 11-14, 21

WiFi Wireless Fidelity. 14 


\section{Chapter 1}

\section{Introduction}

While localization forms one of the most critical aspects of many different problems, it still re-

mains an open problem for indoor environments $[1,2]$. In robotics applications, for instance, there has been many solutions involving different sensors of physical principles proposed to be used for this matter. However, under dynamic, unstructured, and complex nature of the interior, there is no sensor principle solves this problem for all indoor environments. Therefore, it is common to see incorporation of localization results with internal measurements such as the measurements of wheel encoders. This thesis investigates the potential of the use of radio waves for indoor localization purposes.

This section provides a brief explanation of background of the this thesis work followed by the objectives. The summary of contributions will be provided as well as the general outline of the paper. 


\section{$1.1 \quad$ Background}

Indoor environments show challenging circumstances for localization techniques due to complex layout of indoor environments, presence of obstructions, materials used to build the structure of indoor environments, and other limitations imposed by the sensor principles used for localization purposes. In this section these limitations will be discussed in the context of indoor localization, as well as some potential advantages of radio waves over different sensor principles in the indoor localization context.

Indoor environments consist of highly complex layout at their core in comparison to outdoor environments such that the layout creates challenging circumstances for the localization of the agents in the environment. Unlike outdoor environments that can provide Line-of-sight (LOS) measurements about the environment to localize with direct detection of unique features of the environment, indoor environments often display Non-Line-of-sight (N-LOS) measurements due to mentioned complex layout. This limitation in the information

gathering often creates compelling challenges for optical sensors due to wavelength of the signal such sensors rely on. For instance, an optical sensor which employs visible light to capture information about the localization environment cannot gather information behind the walls, around the corners of the environment due to strict LOS dependency of such sensor types. Figure 1.1 depicts the LOS coverage of an indoor environment. As can be seen in the figure, LOS coverage of the localization markers contains a small portion of the environment which impedes sensors which solely rely on LOS propagation to provide reliable measurements about the environment. Along with the obstructions in the localization environment, the materials consist of indoor environments provide more challenging circumstances for different sensor suits. For instance, laser light based sensors tend to provide less precise measurements in the presence of the black obstructions. 


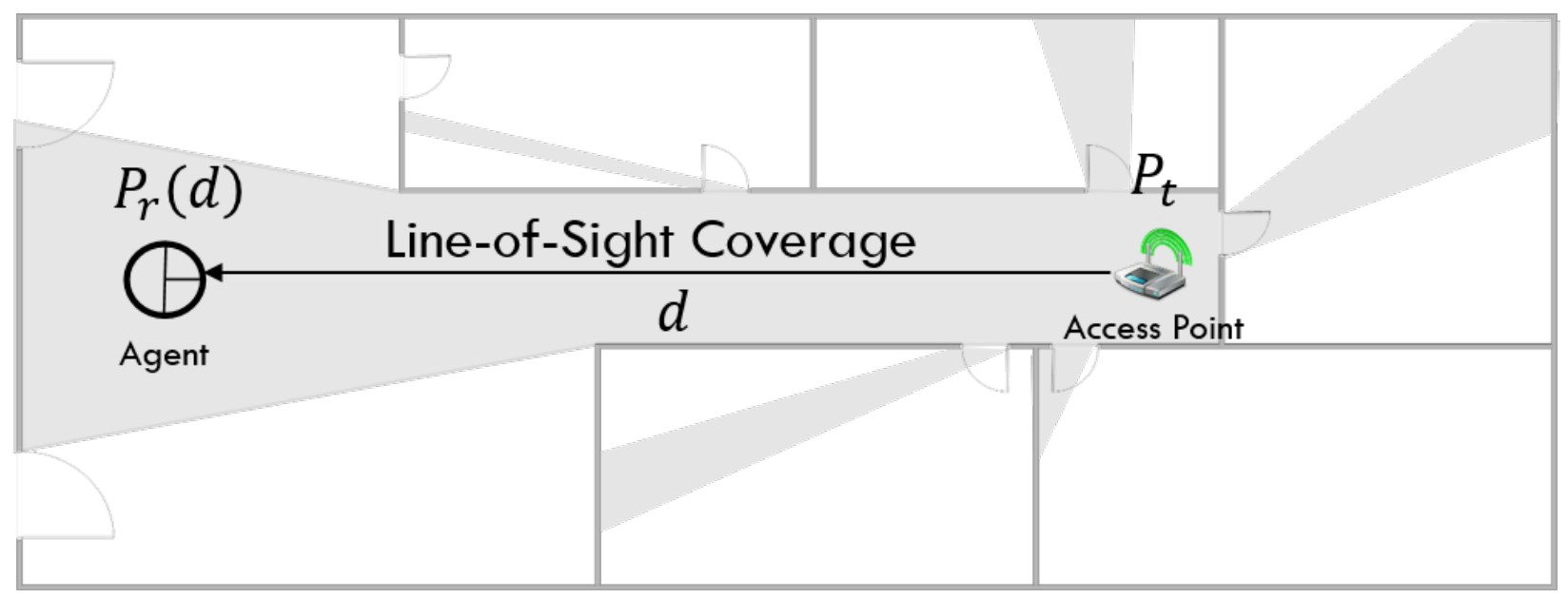

Figure 1.1: LOS Coverage of a generic environment: As can be seen in the figure, the LOS coverage is limited due to the layout of the environment.

Similar to complex layout of indoor environments, the obstructions impedes reliable information gathering about the localization environment. Due to highly dynamic nature of indoor environments where types and distributions of the obstructions vary widely, LOS coverage of the sensor reduces to even smaller portion of the environment. Therefore, sensors which rely on strictly LOS measurements lack ability to localize the agent in the environment in the same extent in comparison to sensors can employ N-LOS measurements.

In addition the limitations imposed by the environment, the sensor principles depending on the physics used to manufacture the sensor create compelling limitations on the localizations. For example, sensors based on laser light tend to provide inaccurate measurements around glass obstructions due to difference in the refractive index of the glass and concrete, a common construction material. Even the curing time of concrete, thickness of the wall partition play significant role in this index governing how light behaves when impinges on such surfaces [3]. Optical sensors, similar to laser light based sensors, tend to provide less accurate measurements where the agent travels in the environment with higher speeds due to motion blur resulting in the gathered images. By increasing the data acquisition frequency, 
motion blur can be prevented, in return of increased bandwidth that the optical sensor needs to use.

\subsection{Radiolocation}

Radiolocation is a localization technique where some characteristics of radio waves are used to determine the position of the target of interest. With the proliferation of the wireless networks, radio waves has received a great amount of interest from the indoor localization research $[4,5,6,7]$. Along with the pervasive availability, radio waves can penetrate in to obstructions on the propagation path, reflect, diffract around the corners, resulting in N-LOS propagation. Therefore, radio waves do not require direct LOS propagation in order to determine the location of the agent in an indoor environment. This ability of the radio waves creates a great potential for localization purposes because of the limited LOS area which can be provided indoor environments.

The power of communication ability even in the presence obstacles introduces some challenges in the determination of the agents in the environment because the radio waves behave differently along different propagation paths. For instance, LOS propagation shows a log-linear power decay as the distance between the access points and the agent increases. In the case of N-LOS propagation, however, different propagation mechanisms, namely, reflection, diffraction and scattering take place. These propagation mechanisms introduce multipath effect where the different copies of the same transmitted signal is received by the agent with different phase delay due to the different propagation paths. This phenomena eventually degrades overall localization performance. As such many indoor radio wave propagation models for different propagation models have been introduced $[8,9,10,11]$, because the success of indoor radiolocation techniques can be mainly attributed to the accuracy of 
the indoor propagation model characterizing the radio wave propagation in the environment.

Among all the empirical models, Log-distance Path Loss (LDPL) [12], which implicitly characterizes the attenuation caused by the obstructions in the environment, has been most popularly used for its strength in accuracy. However, LDPL and its variants model the propagation of the radio waves in a global manner, where the attenuation of the waves is modeled as a function of the distance between two antennas. This raises the question: "How would LDPL behave where the attenuation sources are not uniformly distributed over the localization environment?" In the mentioned case, the same distance between two antennas would not result in the same Received Signal Strength (RSS) fingerprints, if the propagation paths contain different levels of obstructions. In a typical floor plan depicted in Figure 1.2, for instance, a fairly empty area on the west of the floor results in LOS propagation path while the hallway shows N-LOS propagation characteristics. Employing a global propagation model would result in characterization of the disturbances of storage area in the same extent that of the loading dock. Therefore, it is crucial to build an accurate attenuation profile of the environment so that the difference in LOS and N-LOS propagations can be characterized precisely.

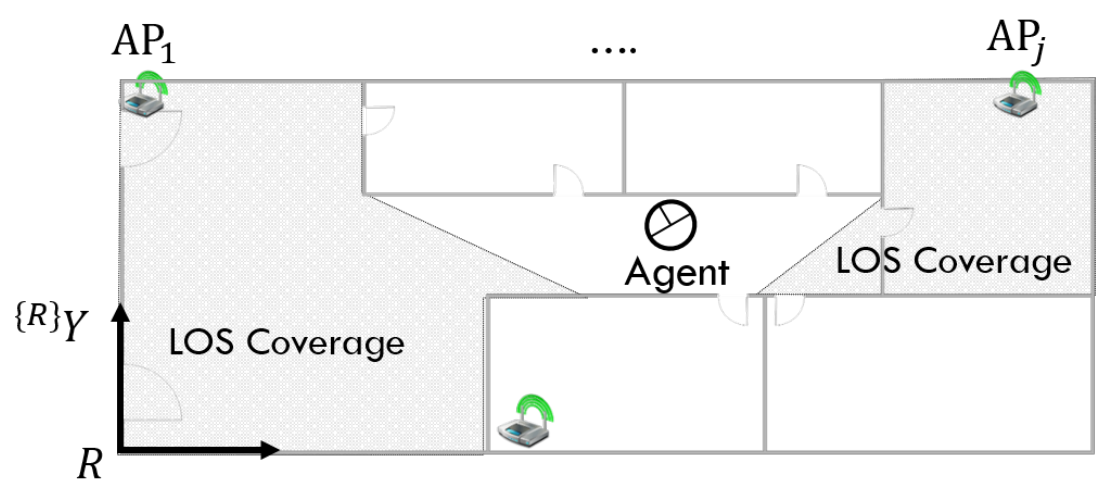

Figure 1.2: A typical floor plan of an indoor environment: The propagation pattern of the access points often yield vast NLOS areas in the region resulting in multi-path propagation. In such scenarios, a propagation model which characterizes the propagation patterns globally might result in inaccurate radiolocation results. 


\section{$1.3 \quad$ Objectives}

The global approximation of radio wave propagation gives a rise to an open problem where attenuation of a small region deviates the whole propagation model. For this reason, this thesis seeks to enhance the existing path loss models by characterizing the irregularities of the environment as a function of location, while keeping the hardware architecture uncomplicated.

In the light of this considerations, the objectives of this thesis is to:

1. develop a new propagation model which can characterize the radio wave characteristics locally,

2. develop a radiolocation technique based on the new propagation model

3. validate of the proposed technique

\subsection{A Grid-based Indoor Localization Technique based on Spatially Coherent Path Loss Model}

This paper proposes a grid-based indoor radiolocation technique based on the new path loss model called Spatially Coherent Path Loss Model (SCPL). RSS fingerprints are first collected at known locations in the localization environment. SCPL uniformly divides the environment into grid-cells, and the collected fingerprints are then assigned to corresponding grid-cells. For each grid-cell, the model parameters are estimated with the fingerprints collected within the grid cell. Unlike the conventional LDPL based radiolocation techniques, which characterize the wave propagation globally as a function of distance between transmitting and receiving antennas, SCPL employs local propagation modeling by employing 
these parameters which characterize the radio wave propagation with explicit attenuation terms for each grid cell. Therefore, the impact of the nonuniform distribution of attenuation sources in the environment can be minimized. Given an RSS fingerprint collected at an unknown location, the target location is determined by employing the approximated wave propagation and trilateration. The grid-cell representation of the environment is then further incorporated into localization by representing the results as probability distribution over the grid-cells. As a result of characterization of radio wave propagation for each grid-cell, SCPL is able to model the irregular attenuation of the environment locally. Furthermore, SCPL requires only one receiving antenna in order to localize the target of interest; therefore, it can work with commodity radio modules as well as different frequency bands.

\subsection{Summary of Contributions}

In this section, a summary of the contributions will be provided.

1. Development of a new path loss model:

Under the light of the objectives, development of a new radio wave path loss model which can tackle N-LOS propagation and resulting multipath effect on the propagation forms the basis of the contributions. As introduced in the previous section, SCPL is able to locally model radio wave propagation with its parameters which explicitly characterize the attenuation of each local region individually. By doing so, effects of obstructions in a small region would carry over to global model.

2. Development of an indoor localization technique based on the new path loss model:

As mentioned earlier, LOS coverage of the sensors which are commonly used for localization purposes is limited in indoor environments. Radio waves, due to their ability 
to propagate even under N-LOS conditions, are good candidate technology for indoor localization purposes, if attenuation results from N-LOS propagation can be characterized. Therefore, development of a new radiolocation technique based SCPL, which can locally model attenuation sources, forms the second basis of the original contributions of this thesis study.

3. Validation of the proposed technique with experimental study:

An in-depth analysis of the the proposed path loss model, as well as the proposed localization technique, forms the third basis of the original contributions of this thesis study.

4. Employment of existing wireless sensor networks:

The proposed localization technique employs commodity radios used in the existing wireless networks. This ability of the proposed technique adds value to the existing infrastructure at no cost and provides a potential scalability where many agents need to be localized. Along with the cost and scalability, the use of radio waves provides a great potential of increase in localization performance where multipath effect is observed. This potential inherently enables the proposed system to be employed in indoor environments where LOS coverage is often limited. Therefore, sensor placement in an environment would not be a concern of design in the indoor localization problems.

\subsection{Outline}

The organization of this thesis is as follows:

- Chapter 2 describes the past effort for radiolocation techniques 
- Chapter 3 gives a brief overview of a radiolocation technique based on a path loss model

- Chapter 4 presents the details of the proposed technique

- Chapter 5 specifies the experimentation conducted

- Chapter 6 summarizes the conclusion and future work of this thesis

\subsection{Summary}

This chapter provided an brief introduction to the radiolocation problems and the common problems of using radio waves in the context of localization. A need for a new propagation model for indoor localization problems was then established. A new indoor localization technique based on a new path path loss model was then introduced as well the objectives. Considering the objectives, the original contributions of this thesis were laid out. Consequently, the layout of describing the remaining chapters of this thesis is presented. 


\section{Chapter 2}

\section{Literature Review}

This chapter provides a survey of indoor radiolocation techniques in the literature. The indoor radiolocation techniques can be classified into four main stream of research camps based on the characteristics of the radio wave used for localization purposes. This chapter provides a brief overview radiolocation techniques based on time, power, angle measurements, and channel properties as well as the the principles of each technique. Figure 2.1 demonstrates the classification of the past work based on the measurements employed in the localization scheme. 


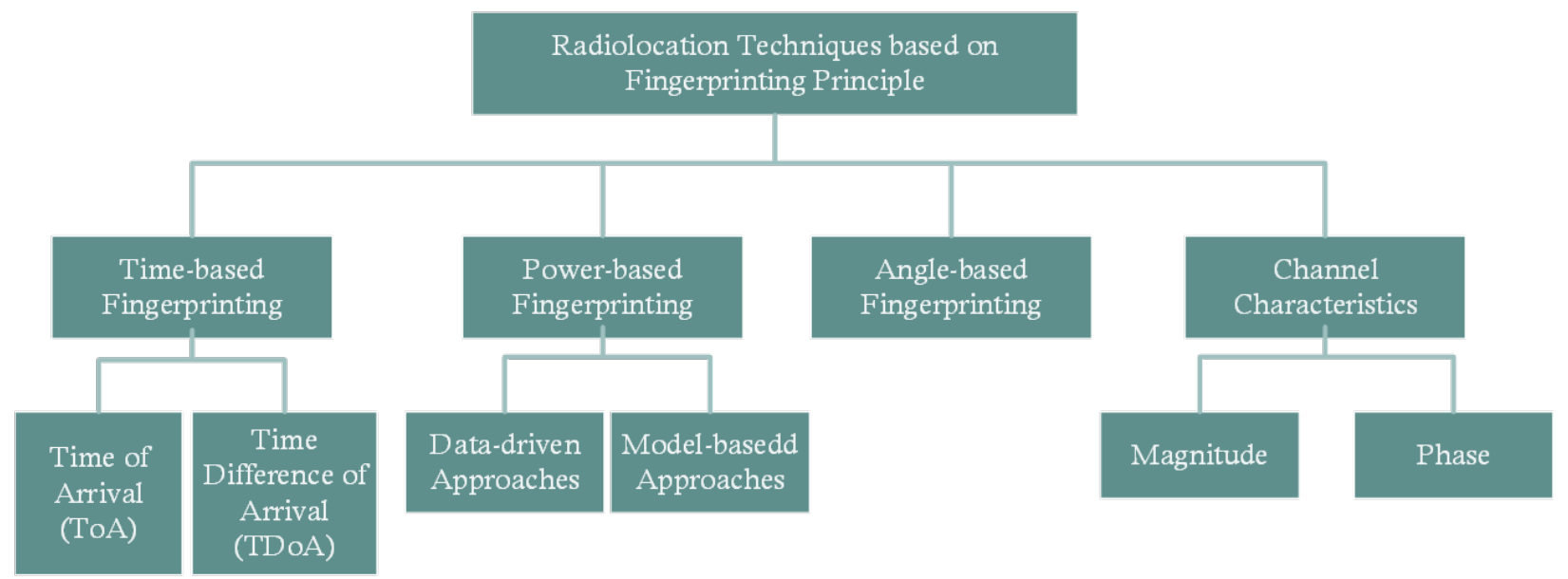

Figure 2.1: Radiolocation Techniques based on Measurements Employed

\subsection{Radiolocation Techniques based on Time-based Mea- surements}

Temporal characteristics of received radio wave is one of the most common fingerprint employed in various radiolocation techniques. Time-based measurements of radio waves are generally used to estimate the propagation path by measuring the time difference between the emission and the reception of the signal. These measurements can be mainly divided into two main categories: Time of Arrival (ToA) and Time Difference of Arrival (TDoA). ToA measurements are used to directly estimate the length of propagation path by using propagation duration between the transmitting and receiving antennas, while TDoA employs the difference in propagation duration of different radio waves emitted by different access points. Each of these measurement techniques has its own advantages and disadvantages; in this section, these temporal characteristics of radio waves are investigated in the context of indoor localization. 
Time of Arrival-based Radiolocation The propagation duration of a radio wave can be used to determine the length of the propagation path. ToA measurements estimate the location of the agent by measuring the time duration of the radio waves to propagate from the access points to the agent. Since radio waves travel in an indoor environment with a known speed, i.e. the speed of light, the duration of propagation linearly correlates with the propagation path. By individually estimating the length of the radio propagation path radio waves transmitted by different access points, the location of the agent can be fixed relative to the each access point. Section 2.1 represents a generic radiolocation technique with ToA measurements.
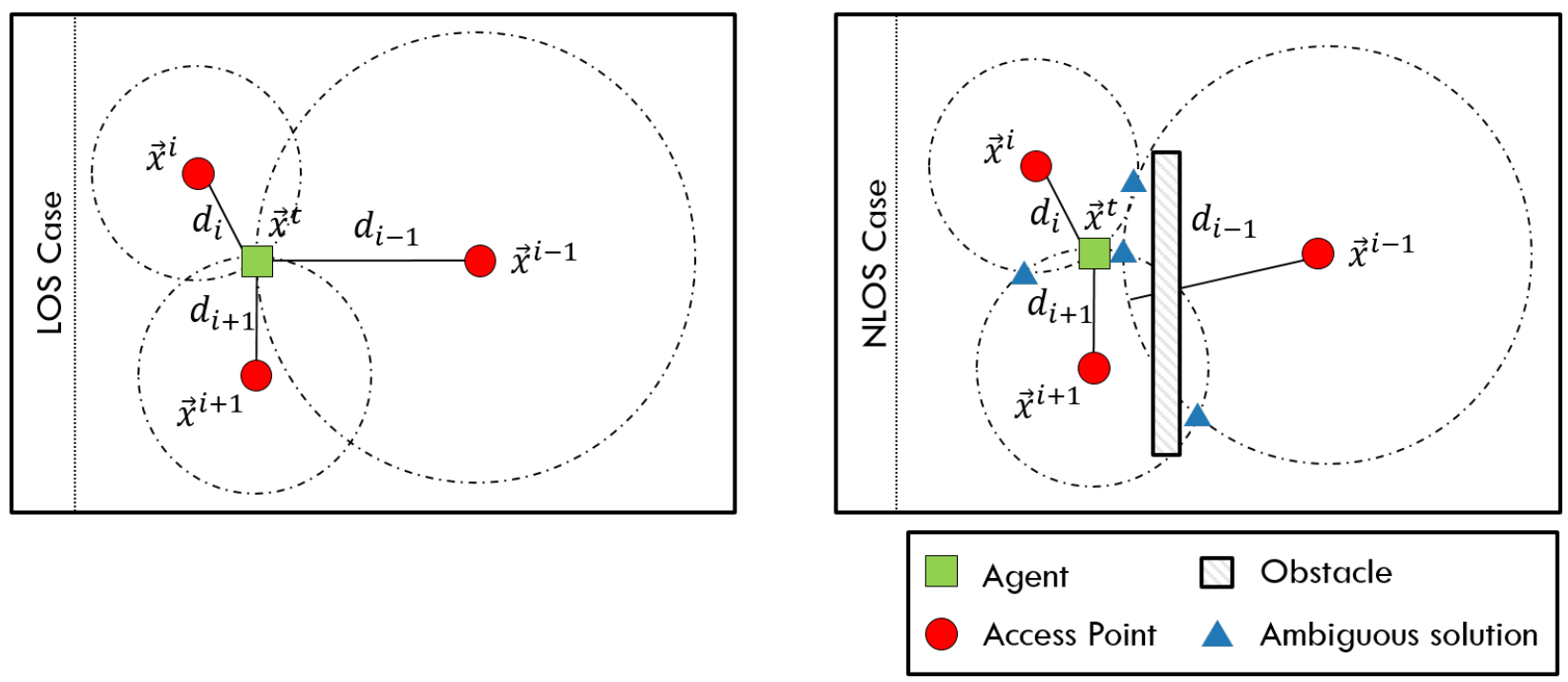

Figure 2.2: Localization of the target with time of arrival fingerprints under LOS (left) and N-LOS (right) propagation. Each time-of-arrival measurement induces a sphere centered at the access point. As can be seen in the figure on right, NLOS propagation conditions often result in ambiguous solutions.

Time Difference of Arrival-based Radiolocation Similar to ToA measurements, TDoAbased measurements employ the propagation time between the access points and the agent. In TDoA measurements, however, the time difference of arrival from each access points constitutes the main scheme of the localization technique. Section 2.1 represents a generic 
radiolocation technique with TDoA measurements.
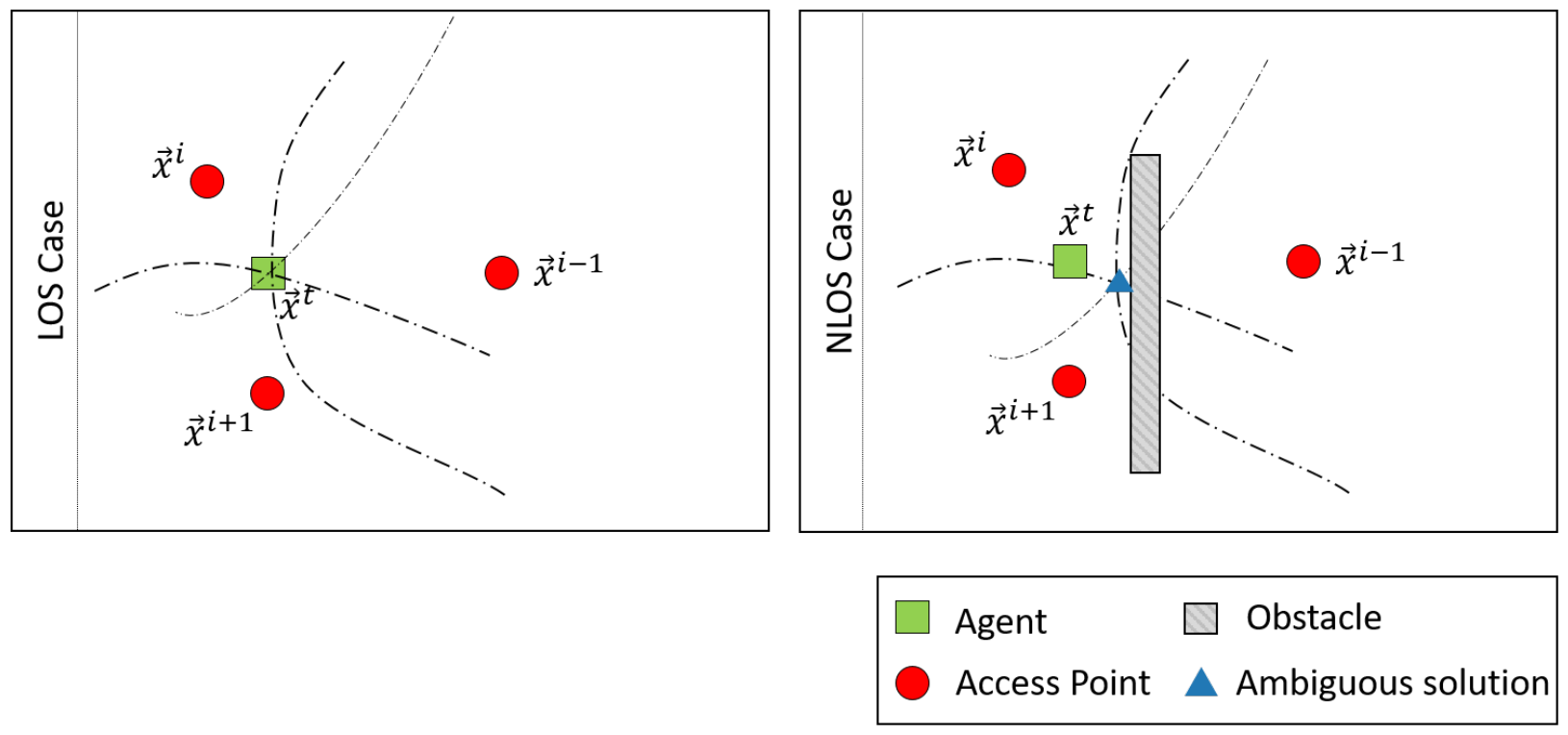

Figure 2.3: Localization of the target with time difference of arrival fingerprints under LOS (left) and N-LOS (right) propagation. Each time-difference-of-arrival measurement induces a hyperbola between two access points. As can be seen in the figure on the right, NLOS measurements results in inaccurate estimation of hyperbolae resulting in ambigious or inaccurate solutions.

Temporal characteristics of radio waves often yields an accurate estimation for length of propagation path, if the propagation path is direct LOS. Radiolocation systems based on the temporal characteristics of the radio waves generally suffer from two major problems: multipath effect caused by N-LOS propagation and high precision needed for time measurements. It is rather common in indoor radiolocation systems to observe propagation paths with N-LOS terms due to dynamic nature of indoor environment. Therefore, the estimated path might not correspond to LOS path between access points and the agent, resulting in a severe degradation of location estimation accuracy. Along with the severe affects of N-LOS on ToA-based and TDoA-based measurements, precision of time-synchronization is another major problem. For example, the required time precision needed in one-way communication scheme for decimeter-level localization accuracy is in the order of nanoseconds [13]. 
Denis et al. [14] addressed the N-LOS affects by providing an comparison analysis of a radiolocation system using first-arrival and strongest received signal. Based on their experimental results, the authors suggested the localization accuracy improves with the use of first arrival signal. However, the robustness of first arriving signal detection heavily relies on thresholds distinguishing the transmitted signal from the background noise. Too low thresholds increase the rate of type II errors where background noise is mistaken with the original signal, while too high thresholds result in disregard of the original signal [15].

While many different statistical tools have been employed in the detection of N-LOS measurements such as normality tests [16], residual tests [17, 18], Borras et al. [19] focused on active N-LOS detection from the resulting range estimations of their radio-based measurement system. In their system, a log of previous estimations are kept and fit a Gaussian distribution. If a new estimation deviates from the distribution describing the estimation history, it is assumed to be a N-LOS measurement.

In order to address highly accurate time precision need for ToA measurements, Vashihst et al. [20] exploited the fact that modern Wireless Fidelity (WiFi) devices span multiple frequency bands scattered around $2.4 \mathrm{GHz}$ and $5 \mathrm{GHz}$. The proposed technique measures ToA across different frequency bands by making transmitter radios, i.e. access points, and the receiver radio placed on the agent transmit and receive signal on different frequency bands. The measurements were then stitched together to accurately estimate the ToA. In order to tackle synchronization problem, two communication is often used. The agent interrogates all the access points by issuing a broadcast signal in the network, while the access points immediately respond this interrogation. Kossel et al. [21] elaborated this scheme with backscatter modulation with frequency hopping. In their work, the interrogation signal is coherently reflected back to the agent by the access point, while superimposed with a specific modulation. This backscatter modulation is an essential solution for time synchronization; however, the 
interrogation signal needs to travel the propagation twice in order to be received back by the agent. Hence, the propagation loss significantly increases which might result in the original signal to be overwhelmed by the background noise.

\subsection{Radiolocation Techniques based on Power-based Measurements}

Unlike temporal-characteristics of radio waves, power-based measurements do not assume LOS propagation path. For that reason, despite their lower localization accuracy in LOS conditions, power-based measurements are usually preferred than time-based measurements in adverse multipath conditions. The power-based measurements are usually employed in mainly two different approaches for localization purposes. The data-driven approaches use pairs of RSS fingerprints with corresponding positions to estimate an implicit localization function which minimizes the localization error across the training data from which the localization function is estimated. On the other hand, model-based approaches estimate some parameters of a propagation model, which is based on physical constraints of the radio waves, from the collected RSS fingerprints. This section briefly covers existing approaches for both data-driven and model-based radiolocation techniques.

\subsubsection{Data-driven Approaches}

Data-driven radiolocation approaches employ RSS fingerprints and their corresponding location labels collected in an indoor environment to approximate a localization function. These approaches are often consisted of two main stages in their core: offline and online [22, 4]. In the offline stage, RSS fingerprints are collected at known positions. These fingerprints and 
their corresponding locations are kept as a priori. In the online stage, the agent collects a tuple of RSS fingerprints from different access points at an unknown location, and identifies the most matched fingerprint in the priori information to infer its location. Figure 2.4 represents a generic data-driven radiolocation approach in detail.

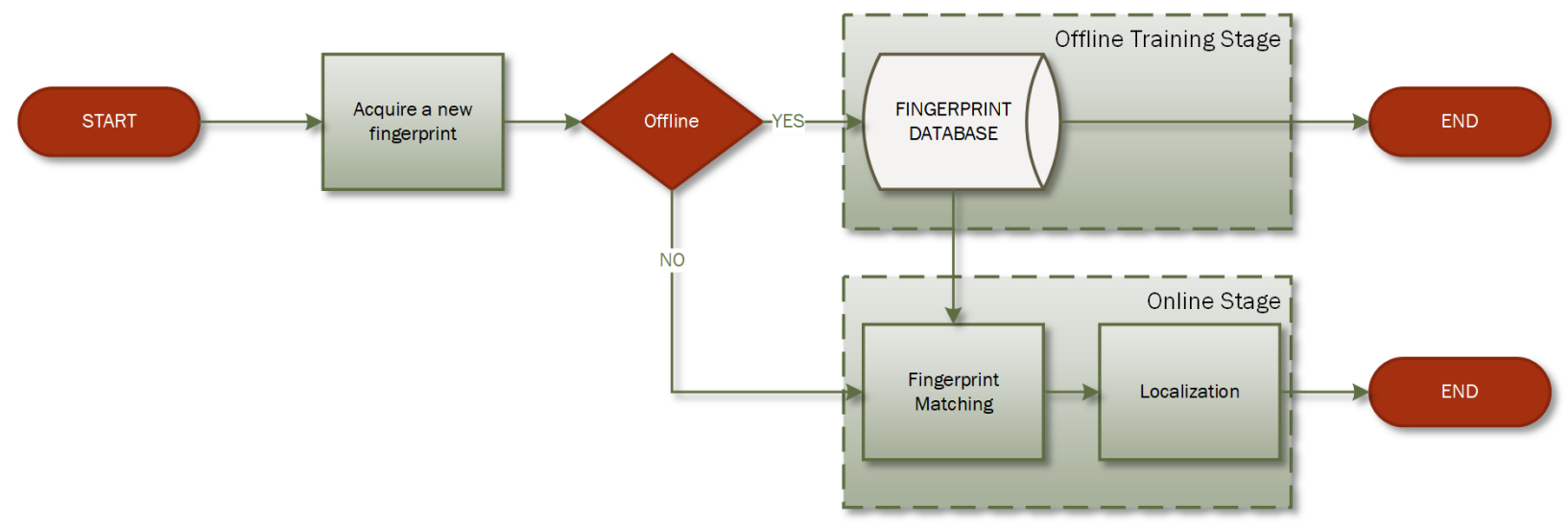

Figure 2.4: Data-driven Fingerprinting-based Radiolocation Approach

The data-driven approaches are also known to be model-free approaches because of their ability to represent the relationship between fingerprints and the locations, not constrained by any physical law. This relation is often determined by estimation of a localization function mapping fingerprints to locations with minimum error. This function is often obtained with different supervised learning algorithms. While not being constrained by any physical model, the generalization ability of these approaches is often bounded by the amount and quality of collected data, and selection of training locations. Therefore, missing values or high noise in collected RSS fingerprints affects the overall localization significantly.

One of the earliest data-driven approach is proposed by Bahl et al. [23]. In their work, RSS fingerprints collected from different access points, were represented with their mean and standard deviation for each location, constituting the fingerprint database. In the online stage, RSS fingerprints collected at unknown location were matched with a k-NN based matching algorithm to determine the location of the agent. Their k-NN implementation 
employs a weighted average of $k$ number of nearest neighbors of the novel fingerprint in the fingerprint database. A drawback of k-NN based localization radiolocation techniques is that $\mathrm{k}-\mathrm{NN}$ requires to store all the fingerprint database.

Missing values in RSS fingerprints degrades the localization performance significantly. To this end, Support Vector Machines (SVM) have been popularly used with different kernel functions to tackle the fingerprint uncertainty and incomplete fingerprints. Wu et al. [24] employed a SVM-based regression approach based on a modified ANOVA kernel to handle sparse RSS fingerprints to reduce the impact of the missing values in collected RSS measurements on the localization performance. Youssef et al. [25] employed a statistical approach where measurements of an access point as a random variable over time and localization environment. Instead of using raw RSS fingerprints, this preprocessing scheme enabled their system to handle to address missing values and high uncertainty of fingerprints. In offline stage, their approach groups fingerprints of each access points in the clustering module, followed by a correlation analysis. In the offline stage, the Discrete Space Estimator matches the collected fingerprint to a training fingerprint maximizing localization probability, followed by the Continuous Space Estimator which incorporates the continuity of a human movement.

Fahed et al. [26], on the other hand, focused on time-varying nature of communication channels. Their radiolocation system employs a dynamic neural network in order to reduce the impact of the fluctuations in the collected RSS fingerprints. The neural network was consisted of one hidden layer with log-sigmoid activation function, while the output layer was consisted of two units which was succeed by a linear activation function. This model receives RSS fingerprints with time delay to tackle with time-varying nature of the radio channels. Their results suggests that such network designs are able to reduce severe effect resulted by the motion of the agent itself. 
Instead of conventional regression-based approaches, Trawiński et al. [27] considered indoor radiolocation as a classification problem. Their system consists of many fuzzy-rule based classifiers trained individually on the RSS fingerprints collected during the offline stage. The result of each classifier is then filtered, and finally fused into final location result. By employing many classifiers, and post-filtering, their system is able to tackle high uncertainty of RSS fingerprints.

\subsubsection{Model-based Approaches}

Model-based radiolocation approaches exploit the physical constraints of the radio waves to characterize the radio wave propagation. Radio wave propagation follows Maxwell's equations, while exact solution for these equations are often difficult to obtain due to dynamic nature of the indoor environments. However, these equations can be generalized for specific propagation mechanisms in order to obtain empirical solutions. Model-based radiolocation techniques usually employ these empirical approximations for physical constrains imposed by Maxwell's equations. LDPL is a generic propagation model which describes the radio wave propagation in terms of attenuation as a function of distance between two antennas.

In model-based approaches, the model parameters characterizing the propagation characteristics are first estimated from RSS fingerprints collected different locations in an indoor environment. Once the radio wave propagation is characterized with the estimated parameters, the location of the agent can be determined with a RSS fingerprint and the model.

Figure 2.5 represents a generic data-driven radiolocation approach in detail. 


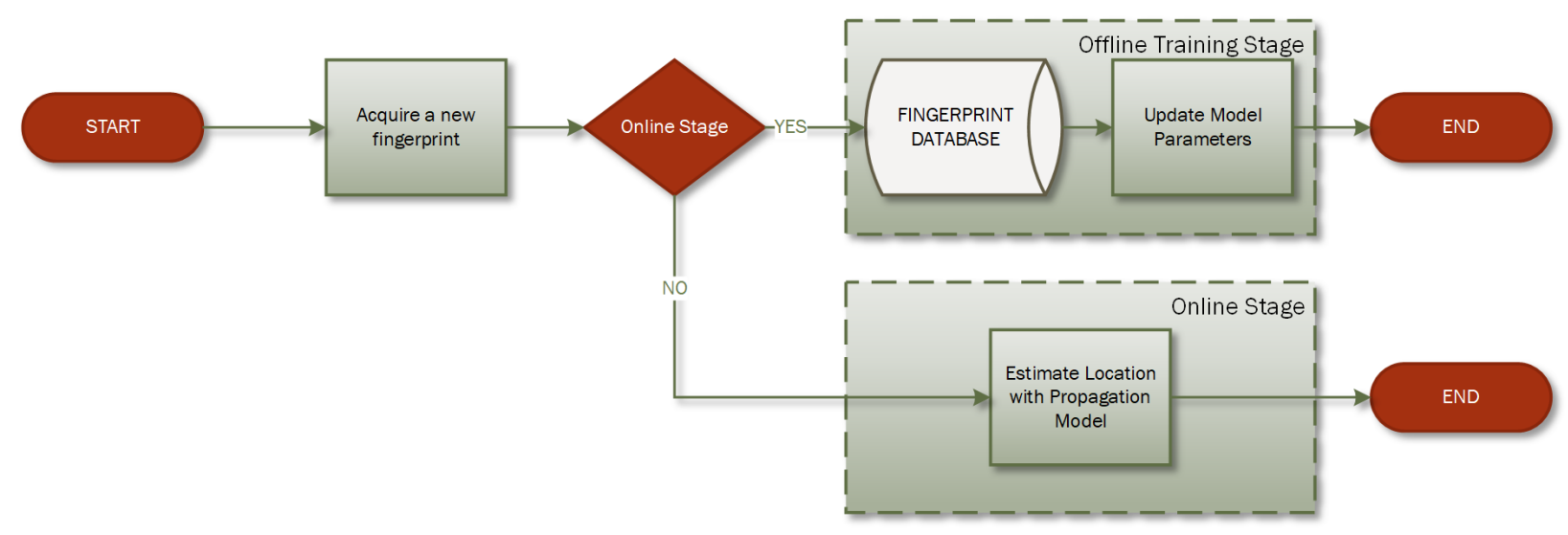

Figure 2.5: Model-based Fingerprinting Radiolocation Approach

In their pioneering work, Bahl et al. [23] employed an LDPL variant in their radiolocation technique and explicitly modeled the attenuation caused by walls in the environment along with the implicit attenuation term. Their technique was employed with trilateration to globally determine the location of the agent. In their later work [28], the authors further modeled environment-caused attenuation, such as the number of people in the environment, by dynamically choosing different set of model parameters. Lim et al. [29] also focused on similar attenuation sources and employed Singular Value Decomposition (SVD) on the collected RSS to reduce the disturbances of the environmental attenuation on the fingerprints. Incorporation of explicit attenuation model into LDPL yielded highly accurate localization results for both techniques.

Chintalapudi et al. [30] introduced a Genetic Algorithm based approach for parameter estimation and applied it after localizing the access point positions as well as that of agent. Goswami et al. [31] proposed a learning-based approach for estimation of LDPL model parameters. The collected RSS fingerprints were represented with a Gaussian Mixture Model, and model parameters were estimated with the Expectation Maximization algorithm. Experimental results have validated the reduction of localization uncertainty caused by the proposed parameter estimation. 
It is common to see hybrid radiolocation techniques in the literature which combines different inertial sensors with radiolocation results. Chen et al. [32] proposed a radiolocation system which is implemented on a smart phone. The inertial measurements of the smart phone is used in the estimation of a human the stride as well as identification of some structural landmarks of the environment such as turns, elevators, escalators etc. The step count of the person and the identified landmarks are used in a dead-reckoning scheme. The results of the radiolocation are fused with the dead-reckoning results in order to tackle to drifting nature of the dead-reckoning. The fusion of the information is attained with a Kalman Filter. Evennou et al. [33] proposed an integration method for a LDPL model based radiolocation and inertial navigation system. Similar to previous work, the inertial measurements are fused with a Kalman Filter. The result of the Kalman Filter is then incorporated with the LDPL model based radiolocation results, hence yielding a smooth trajectory. The resulting trajectory angle is then fed into the Kalman Filter where the inertial measurements are fused.

\subsection{Radiolocation Techniques based on Angle of Ar- rival Measurements}

Angle of Arrival (AoA) measurements employ the difference of the reception time of the same signal by different antennas of a radio module.

As the volume of the chips controlling network interfaces and radios gets smaller, the design of the radio modules has been showing a significant change. The new design of radios incorporate antennas, synthesizer and other needed peripheral devices into one chip. This manufacturing enables radios to have more than one antenna, usually referred as Multiple- 
Input Multiple-Output (MIMO), such that the same package transmitted in the network is captured by the antennas individually. The additional information that extra antennas provide can be used to determine the arrival angle of the radio waves.

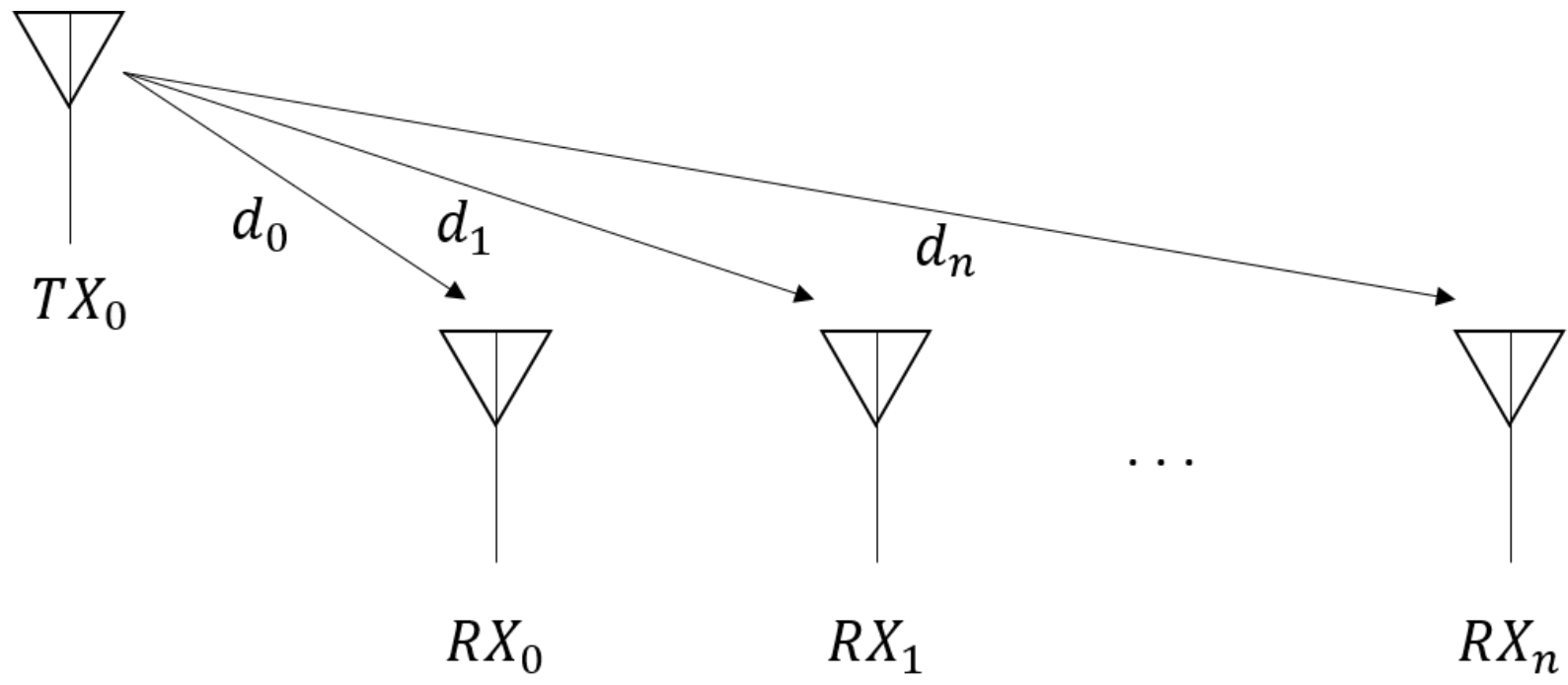

Figure 2.6: Angle-of-Arrival Determination with Multiple Antennas: Since the geometric placement of each antenna is known, the time difference of each received signal is used to estimate the angle of arrival.

Xiong et al. [34] proposed ArrayTrack which uses 16 antennas to estimate AoA. For each detected signal, Arraytrack quickly switches between antennas in order to collect the same signal with different pair of antennas. In order to mitigate the multipath effect, the authors proposed an algorithm which actively disregards reflection paths between the access point and the agent. Finally, Arraytrack employs a maximum likelihood estimation for localization estimation by combining AoA measurements acquired from different access points.

Kotaru et al. [35] proposed a radiolocation technique, employing a commodity radio with three antennas. AoA of each received signal is first estimated with MUSIC algorithm. In order to increase the resolution of AoA estimations, the authors proposed a super-resolution algorithm incorporates ToA into the AoA estimation. By using ToA, the estimation likelihood of LOS direction and the target location is calculated from the collected AoA and ToA 
pairs.

Along with MIMO systems, Synthetic-aperture Radar (SAR) is used in radiolocation techniques to simulate the MIMO design by moving the antenna [36, 37, 38, 39]. The movement of the antenna during the signal reception augments the received information; however, the position of the antenna must be known for each time step with high precision. In order to tackle this precision requirement, Kumar et al. [37] proposed a SAR algorithm which can be used with hand held devices. The radiolocation technique is then combined with the visual cues of the environment provided by a stereoscopic visual localization technique.

\subsection{Radiolocation Techniques based on Channel Char- acteristics}

Channel State Information (CSI) employs higher level information of the radio channel by employing many antennas places on the agent's radio. Unlike other fingerprinting principles which are obtained at MAC layer of the network, CSI is extracted from physical layer (PHY). This fingerprinting principle provides a combined effect of the propagation mechanisms occurred along the propagation path as well as magnitude, phase and frequency of the received signal.

Sen et al. [40] proposed one of the earliest CSI-based radiolocation, called PinLoc, where frequency response of the channel is extracted from CSI fingerprints to enhance the area granularity accuracy. These diverse frequency response of the channel are recorded at different locations, and clustered with Variational Bayesian Inference system into $K$ number

of spots. Once a new CSI fingerprint obtained at an unknown location, it is matched to one of the spots with a log-likelihood metric. 
Xiao et al. [41] proposed FIFS, employing more information from CSI fingerprints, i.e. magnitude and phase, than PinLoc. CSI measurements obtained at an unknown location are first aggregated in the weighted averaging scheme; then matched with training fingerprints with a Pearson correlation. The resulting correlation score is represented with a probability distribution, which is obtained with Bayes' rule, over the localization space.

Later, Wang et al. [42] proposed PhaseFi employing only the phase information obtained from CSI fingerprints. The raw CSI fingerprints are obtained from multiple antennas of the agent's radio, followed by a linear transformation extracting calibrated phase information from CSI fingerprints. The authors proved phase information of CSI fingerprints have a bounded variance, resulting in the reduction of noise in the fingerprints.

In their later work, called DeepFi [43], the authors employed a four layer Restricted Boltzman Machine (RBM) based deep learning algorithm to characterize the localization function by only using the magnitude information extracted from CSI fingerprints. In comparison to FIFS which also employs the magnitude information, DeepFi uses raw CSI fingerprints. The fingerprints collected in offline phase are use to train the RBM structure adopting a greedy learning algorithm, where the deep network is trained layer-by-layer manner followed by a fine-tuning, proposed in [44]. In the online stage, the raw CSI fingerprints are fed into the network to extract stochastic features of the fingerprint. At an unknown location, $n$ number of packets are captured by the agent from which these features are then extracted and fused with Bayes' Rule to infer agent's position.

N-LOS identification and mitigation can be improved by using CSI fingerprints due to the fact that these fingerprints contain richer information about the channel. Cai et al. [45] proposed a technique which employs CSI fingerprints to detect N-LOS signal and mitigate its adverse effect on the localization. Their technique employs a Hampler filter to reject outliers, and a linear transformation to further filter phase measurements. After this preprocessing 
step, a SVM-based binary classifier and regressor are successively employed to detect N-LOS fingerprints, and mitigate their effects, respectively.

CSI fingerprints not only improved overall radiolocation performance, but did also contributed into sever effect of multipath effect. However, these fingerprints require a sophisticated hardware and software architecture combination in their collection. For this reason, it is difficult to employ this fingerprint with commodity radio modules used in wide variety of devices such as Wireless Sensor Networks (WSN)s and Internet of Thing (IoT) devices.

\subsection{Summary}

This chapter provided an overview of the radiolocation techniques based on different radio wave characteristics employed. While significantly accurate under LOS propagation, temporal characteristics of the radio waves are often prone to yielding erroneous estimation under N-LOS propagation. Along with this, the need for clock-synchronization and high precision timing makes temporal characteristics of radio waves less appealing for indoor localization problems. Power-based characteristics of the radio waves were also surveyed. Under LOS propagation, radiolocation techniques which employs power-based characteristics of radio waves often yield less accurate localization results. However, these approaches generally are able to maintain their LOS accuracy in comparison to the techniques falls into former category whose estimation accuracy significantly degrades with N-LOS propagation. Along with power-based fingerprints, AoA fingerprints are also commonly used in radiolocation techniques. While achieving more accurate results, a sophisticated hardware architechture is required to collect these fingerprints. Lastly, radiolocation techniques where characteristics of communication channel carrying the signal was surveyed. Among all, these fingerprints are the least prone to adverse effects of N-LOS propagation, providing accurate localization 
results. However, in order to extract this information, a specific set of hardware and software combination must be used making it difficult to employ the techniques on commodity radio modules. 


\section{Chapter 3}

\section{Radio Wave Propagation in Indoor}

\section{Radiolocation}

This section provides a general framework for radiolocation techniques. In order to provide a complete framework, first the fundamentals of indoor radio wave propagation mechanisms first is laid out. Based upon the formulation of radio wave propagation mechanisms, existing indoor radio wave propagation models are given Consequently, the indoor radiolocation techniques which employs different radio wave propagation models for localization purposes are given, followed by the a summary of the framework concluding the section.

\subsection{Radio Wave Propagation}

The theory of radio wave propagation goes back to James Clerk Maxwell who predicted to existence of the radio waves by proposing the electromagnetic theory of light. Later his work was validated by Heinrich Hertz who demonstrated the physical existence of the radio waves. Since Hertz, the radio waves have been employed in various communication, object 
detection, observation of weather formation and many more applications.

Electromagnetic waves propagate through different mediums where different propagation mechanisms take place depending on the propagation conditions. In this part of the section, these propagation mechanisms are provided. First, the fundamental relation between transmitted and received radio waves is be given, followed by the propagation mechanisms explaining the different types of behavior when radio waves changes propagation medium.

The propagation characteristics of radio waves can be formulated and understood with Maxwell's famous equations. However these equations require a full understanding about all aspects of the propagation, namely, antenna properties, propagation medium, obstructions, radio wave characteristics. In the case of radiolocation techniques, these factors are almost never possible completely to know, measure or characterize.

Due to the complexity of the real environments, it is common in the literature to reverse formulate the propagation of the radio waves by creating a relation transmitted and received signals. Equation (3.1) represents radio wave transmitted with an antenna in an environment.

$$
\begin{aligned}
& s(t)=\mathfrak{R}\left\{u(t) e^{j\left(2 \pi f_{c}+\phi_{0}\right)}\right\} \\
& s(t)=x(t) \cos \left(2 \pi f_{c}+\phi_{0}\right)-y(t) \sin \left(2 \pi f_{c}+\phi_{0}\right)
\end{aligned}
$$

where $u(t)=x(t)+j y(t)$ is a complex baseband signal with $f_{c}$ carrier frequency and $\phi_{0}$ phase delay. The baseband is consisted of two components: in-phase component $x(t)=\mathfrak{R}\{u(t)\}$ and quadrature component $y(t)=\mathfrak{I}\{u(t)\}$.

The signal representing the radio waves can be considered as real signals due to the fact that radio modules modulate and demodulate waves using oscillators which generate 
real sinusoids. However, $s(t)$ is often represented as complex exponential due to the complex nature of Fourier transform, which has a significant role in the frequency analysis of the radio wave propagation.

Received signal can be formulated in a similar manner. Equation (3.2) represents the received signal with complex baseband signal $v(t)$ which is depended on the channel through which radio waves propagate.

$$
r(t)=\Re\left\{v(t) e^{j\left(2 \pi f_{c}+\phi_{0}\right)}\right\}
$$

where $v(t)=u(t) * c(t)$, if channel is time-invariant. $c(t)$ is said to be channel state information of the channel which represent the instantaneous impulse response of the channel. By using Equations (3.1) and (3.2), transmitted and received signals can be represented if channel state information is perfectly known. However, similar to the case of Maxwell's equations, channel state information is difficult to predict or model.

Due to the bottleneck caused by the channel state information, transmitted and received power levels are often used in the context of radiolocation rather than mentioned equations. The power levels of transmitted and received signals can be obtained with Parseval's theorem.

\subsection{Radio Wave Propagation Mechanisms}

In this section of the thesis, details of radio wave propagation mechanisms are provided. As mentioned in the previous section, the channel state information makes it difficult to correlate the transmitted and received signal. Therefore, it is a common practice in literature to characterize radio wave propagation with empirical models. In this section, propagation mechanisms of radio waves, namely, free space propagation, reflection (including refraction), 
diffraction and scattering will be formulated.

\subsubsection{Free Path Propagation}

In his work, Harald T. Friis laid out an empirical propagation model for free space propagation which later almost became a standard propagation books [46]. Friis' Free Space Equation (FFSE) considers the propagation occurring between two ideal antennas in a vacuum space with no obstructions in between. In other words, this model is only able to characterize LOS propagation conditions.

$$
r(t)=\mathfrak{R}\left\{\frac{\lambda G_{t} G_{r} e^{\frac{j 2 \pi d}{\lambda}}}{4 \pi d} u(t) e^{j\left(2 \pi f_{c}+\phi_{0}\right)}\right\}
$$

where the model parameters are stated in the table below:

Table 3.1: Parameters of empirical free-space

\begin{tabular}{lll}
\hline Parameter & Description & Unit \\
\hline$G_{t}$ & Transmitting Antenna Gain & - \\
$G_{r}$ & Receiving Antenna Gain & - \\
$\lambda$ & Wavelength of the signal (Please note: $\left.c=\lambda f_{c}\right)$ & meters \\
$d$ & Separation distance between the ideal antennas & meters \\
\hline
\end{tabular}

As can be seen in Equation (3.3), FFSE characterizes the propagation by relating transmitted and received signal power levels as a function of separation distance $d$ which can be stated as below:

$$
P_{r}(d)=\frac{P_{t} G_{t} G_{r} \lambda^{2}}{(4 \pi d)^{2}}[\text { Watts] }
$$


Transmitting Antenna

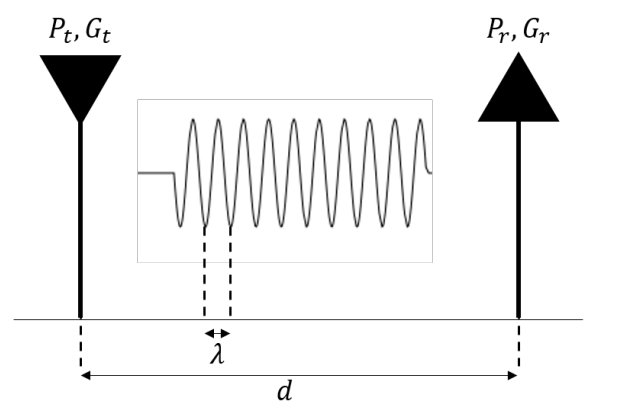

(a) Friis' Free-space equation characterizes propagation between two ideal antennas in a free space as a function of separation distance $d$.

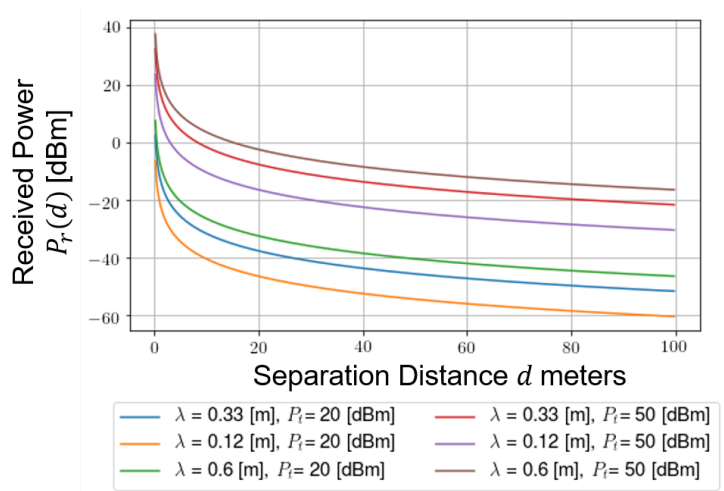

(b) Relationship between transmitted and received power levels.

\section{Figure 3.1: Friis' Free-space Propagation Model}

As shown in Equation (3.4) and Figure 3.1b, the received signal strength falls off inversely proportional to the square of the separation distance. This relation can be seen in Figure 3.2. Due to minuscule level of the power, the transmitted and received power levels are represented in logarithmic scale. Therefore, Equation (3.4) can be written as:

$$
P_{r}(d)=P_{t}(d B m)+10 \log _{10} G_{t} G_{r}+20 \log _{10} \lambda-20 \log _{10} 4 \pi-20 \log _{10} d[\mathrm{dBm}]
$$

By representing the reception power level as Equation (3.5), the received power is represented in logarithmic scale with respect to a milliwatt. Free-space Path Loss can be then defined as a positive gain representing the power loss occurring along the propagation path with the difference between the transmitted and received power in decibel scale.

$$
P L(d)=-10 \log _{10} \frac{G_{t} G_{r} \lambda^{2}}{(4 \pi d)^{2}}
$$




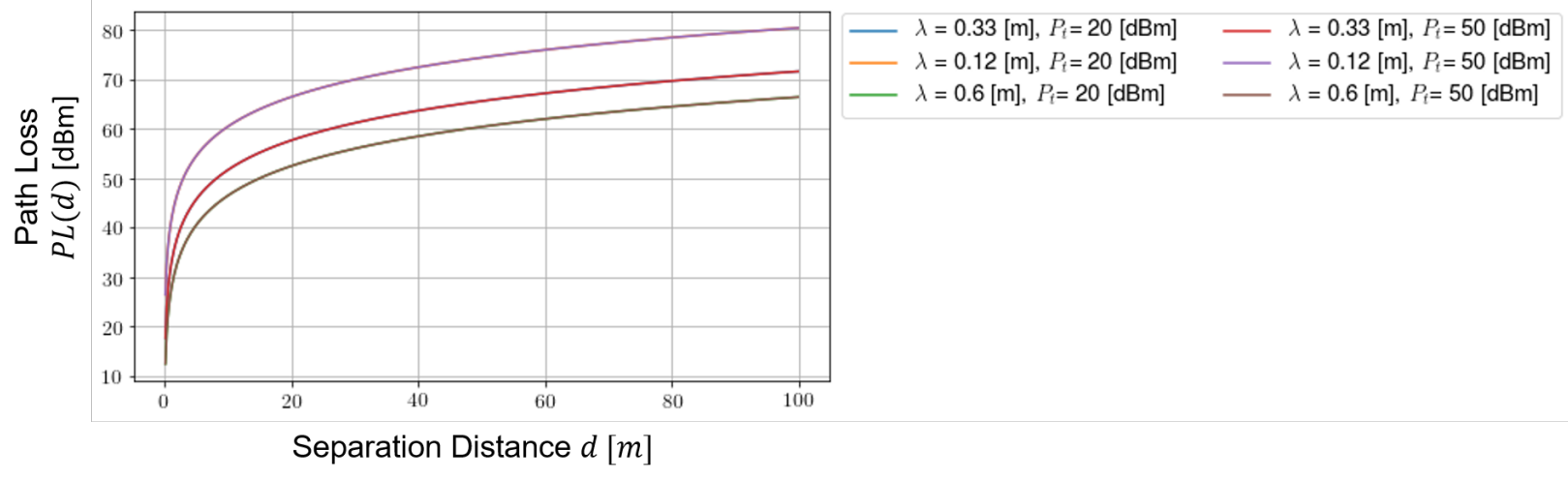

Figure 3.2: Expected Free Space Path Loss as a Function of Distance

\subsubsection{Reflection and Refraction}

In a typical indoor environment, it is safe to assume that direct LOS coverage area of access point contains a limited portion of the environment. Therefore, FFSE is often infeasible to use for indoor radiolocation purposes. However, there are many empirical formulation for reflection mechanism based on FFSE. These models can characterize the multi-path effect resulted by the reflection mechanism; hence increasing the propagation characterization ability of the model. This increase in accuracy to represent the actual propagation and prediction propagation often attributes to localization performance.

The reflection phenomenon occurs if a wave front impinges on a different medium (obstruction) along the propagation path which is bigger than the wavelength of the signal in dimension. Depending on the permeability of the obstruction and the wavelength of the radio waves, some of the radio waves are reflected back, and remaining penetrates into to medium with refraction mechanism. The ratio of the reflected power and refracted power level characterized with refractive index of the obstruction. For instance, metal surfaces behave as a (almost-) perfect reflective medium, while different atmospheric conditions creates significant attenuation for the radio waves [47]. The reflection mechanism can be seen on 
Figure 3.3.

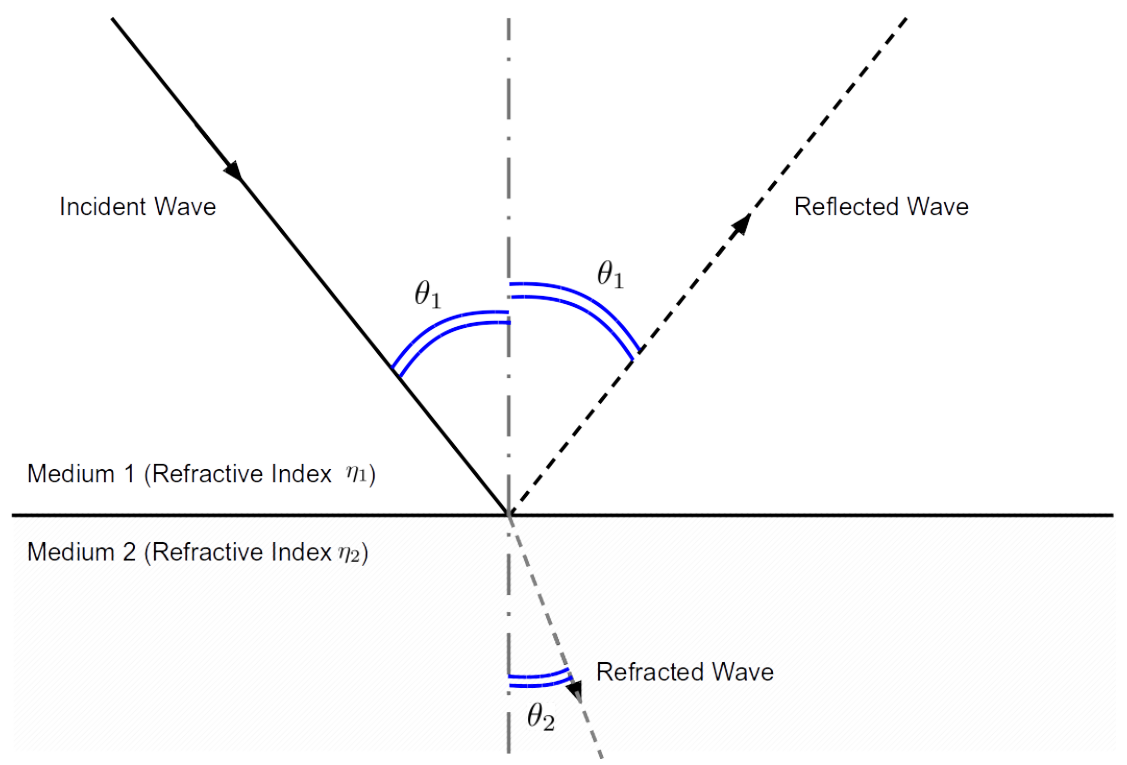

Figure 3.3: Reflection and Refraction of a Radio Wave

\section{Two-Ray Reflection Model}

Two-ray reflection model is popular for open indoor areas where the distance between the access point and the agent is significantly long. In that case, the reflected copies of the transmitted signal from walls and other obstructions arrive at the receiving antenna with a large phase delay, creating multi-path effect. Even though LOS propagation might be established, multi-path effect of these delayed copies changes the induced power at the receiving antenna. Figure 3.4 depicts the two-ray reflection model in greater detail. 


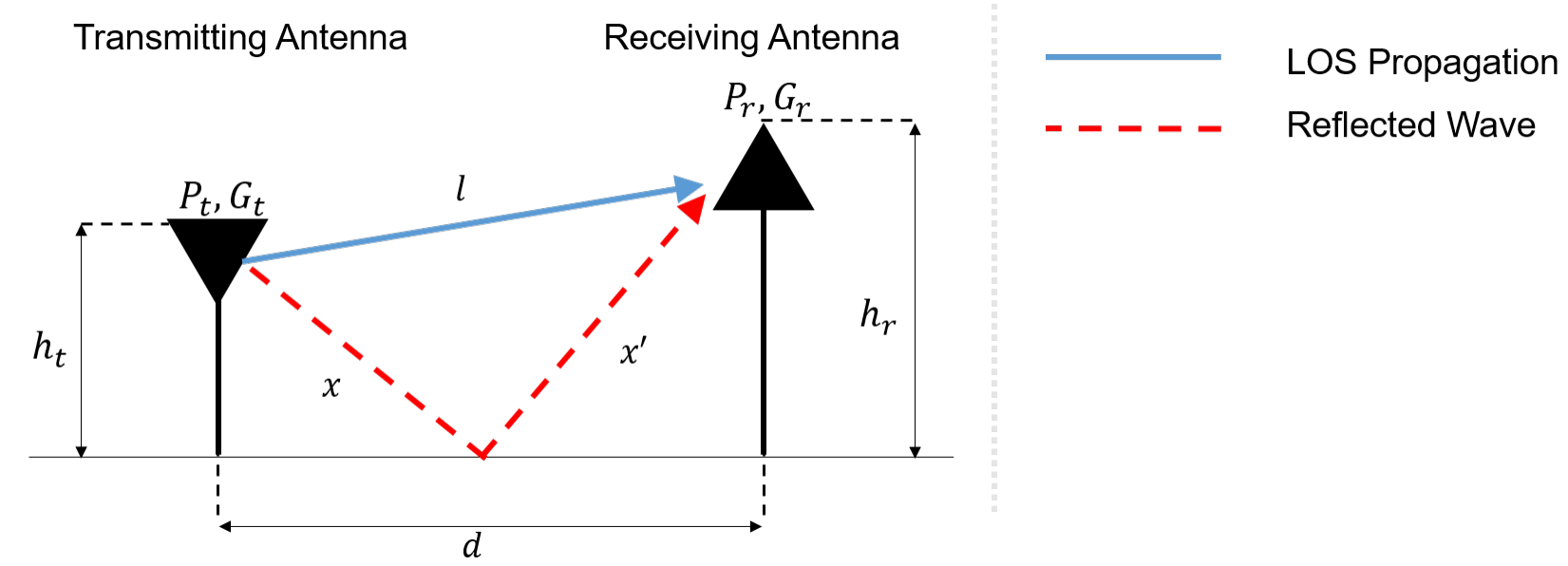

Figure 3.4: Two-ray Reflection Model

As shown in Figure 3.4, Two-ray reflection model can incorporate many aspects of the physical properties of the propagation, such as ground reflection coefficient, height of the antennae, and polarization of the wave. As mentioned earlier, two-ray reflection model assumes, there are two components of the received signal: LOS component and the reflected wave. Therefore, received signal can be expressed as the superposition these two components, which is given in Equation (3.7). As can be seen in the equation of the model, the first component, received wave of LOS propagation path, is represented with FFSE.

$$
r(t)=\mathfrak{R}\left\{\frac{\lambda}{4 \pi}\left[\frac{G_{t} u(t) e^{\frac{j 2 \pi l}{\lambda}}}{l}+\frac{R G_{r} u(t-\tau) e^{\frac{j 2 \pi\left(x+x^{\prime}\right)}{\lambda}}}{x+x^{\prime}}\right] e^{j\left(2 \pi f_{c}+\phi_{0}\right)}\right\}
$$

where $R, \tau, x+x^{\prime}$ and $l$ represent the ground reflection coefficient, delay spread of the signal, reflection path and the LOS path of the signal, respectively. As mentioned earlier, two-ray model assumes a propagation path $l$ such that $x+x^{\prime} \approx l \approx d$. 


$$
\begin{aligned}
& P_{r} \approx\left[\frac{\lambda^{2} G_{t} G_{r}}{(4 \pi d)^{2}}\right]\left[\frac{4 \pi h_{t} h_{r}}{\lambda d}\right]^{2} P_{t} \\
& P_{r}=\left[\frac{G_{t} G_{r}\left(h_{t} h_{r}\right)^{2}}{d^{4}}\right] P_{t}
\end{aligned}
$$

Therefore, the relation between transmitted and received signal strength can be expanded with Taylor series approximation, yielding Equation (3.8).

$$
P_{r}(d)=P_{t}(d B m)+10 \log _{10}\left(G_{t} G_{r}\right)+20 \log _{10}\left(h_{t} h_{r}\right)-40 \log _{10} d
$$

Equation (3.9) represents the transmitted and received power strength of a radio wave under two-ray propagation conditions. As can be seen in the Equation (3.9), received power decays much faster than free-space propagation. While FFSE characterizes the power decay with a inverse relation of squared distance among antennae, two-ray reflection model characterizes with the fourth power of the separation distance. Equivalently, Equation (3.8) can be represented in logarithmic scale. The received power is also independent from the wavelength of the signal. 


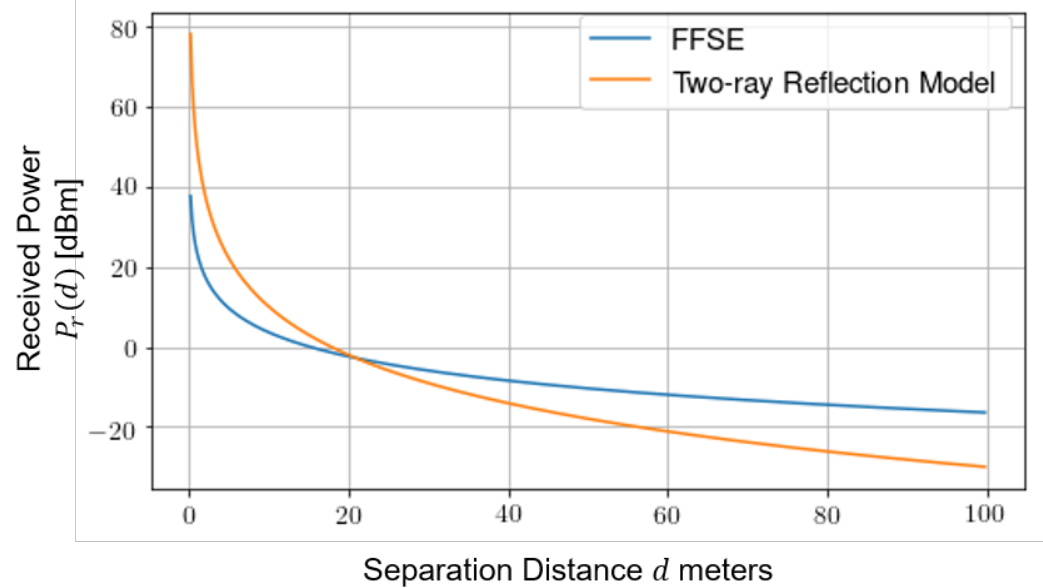

Figure 3.5: Comparison of the received signal levels of Two-ray Reflection Model and Freespace Propagation Model

\subsubsection{Diffraction}

This section of the thesis defines diffraction mechanism and provides a common model characterizing the diffracted radio waves in the context of radio wave propagation. As mentioned earlier, LOS coverage of radio waves in indoor environments is said to be limited. However, thanks to N-LOS propagation mechanism, radio waves can propagate in an environment even in the presence of the obstructions. This ability to propagate under N-LOS conditions provides a great ability for radio waves to be employed in wireless devices for different purposes such as communication and broadcasting TV and radio signals, where optical range of the electromagnetic spectrum cannot be employed because of their strict LOS-only propagation ability. However, this ability comes with a price. If radio waves complete their propagation under N-LOS conditions by reflecting, diffracted and scattering, the received signal is often distorted. Therefore, it is imperative to characterize the magnitude of this distortion effect to successfully employ radio waves for localization purposes.

Diffraction mechanism occurs when radio waves impinges on a sharp corner or surface. 
In this case, radio waves tends to bend around the sharp surface and creates a new wave front. Figure 3.6 represents the diffraction mechanism. The blue arrow on the figure represents the LOS propagation path, while red line represents the new wave-front created by the diffracted radio waves.

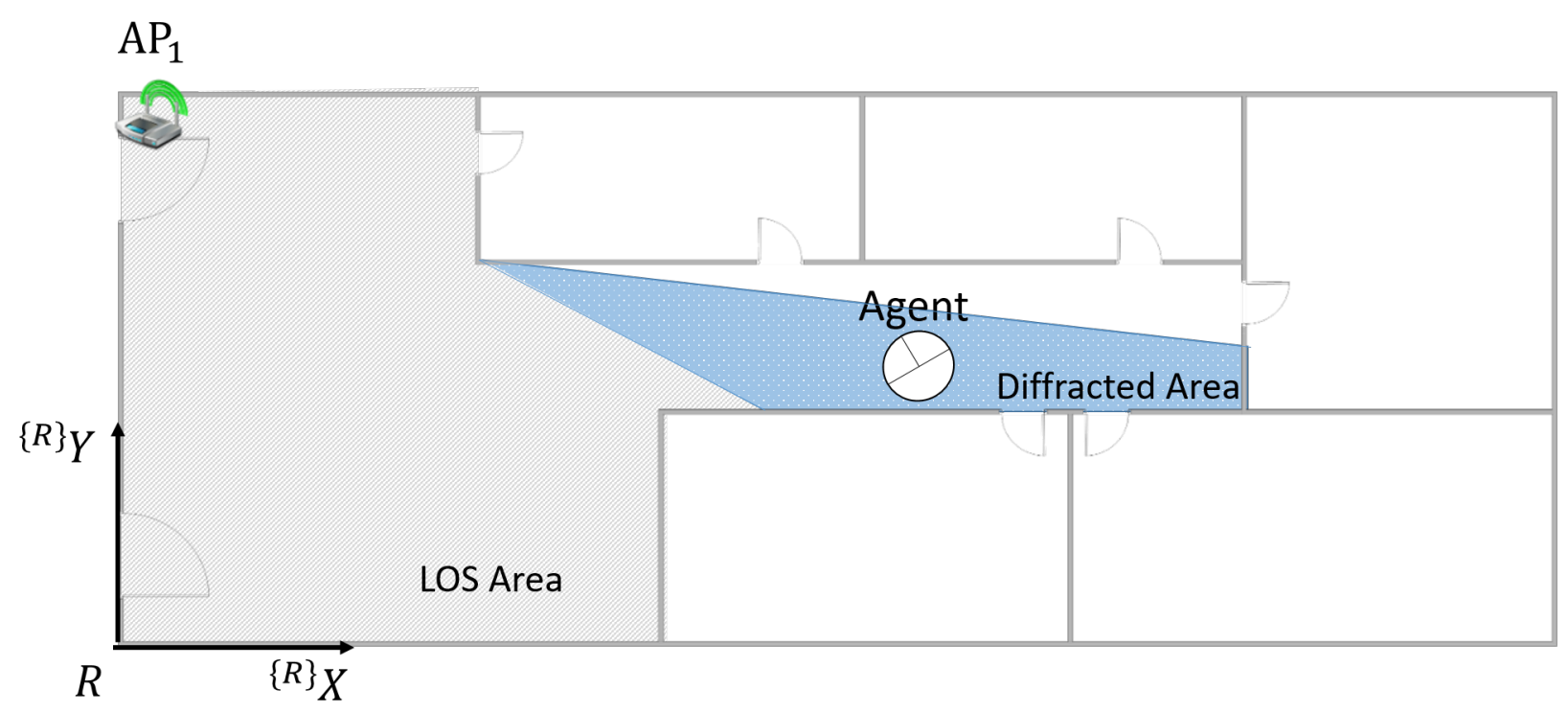

Figure 3.6: Diffraction mechanism in an indoor environment: Diffraction mechanism takes place in indoor environments due to sharp corners and edges of the infrastructures.

\section{Fresnel Knife Edge Diffraction Model}

Fresnel Knife Edge diffraction model is a simplified approach for diffraction phenomenon. This model characterizes the diffracted wave-fronts by using the geometrical theory of diffraction and assumes the obstruction is a wedge rather than general shape. Figure 3.7 demonstrates the details of the Fresnel Knife Edge Diffraction model. 


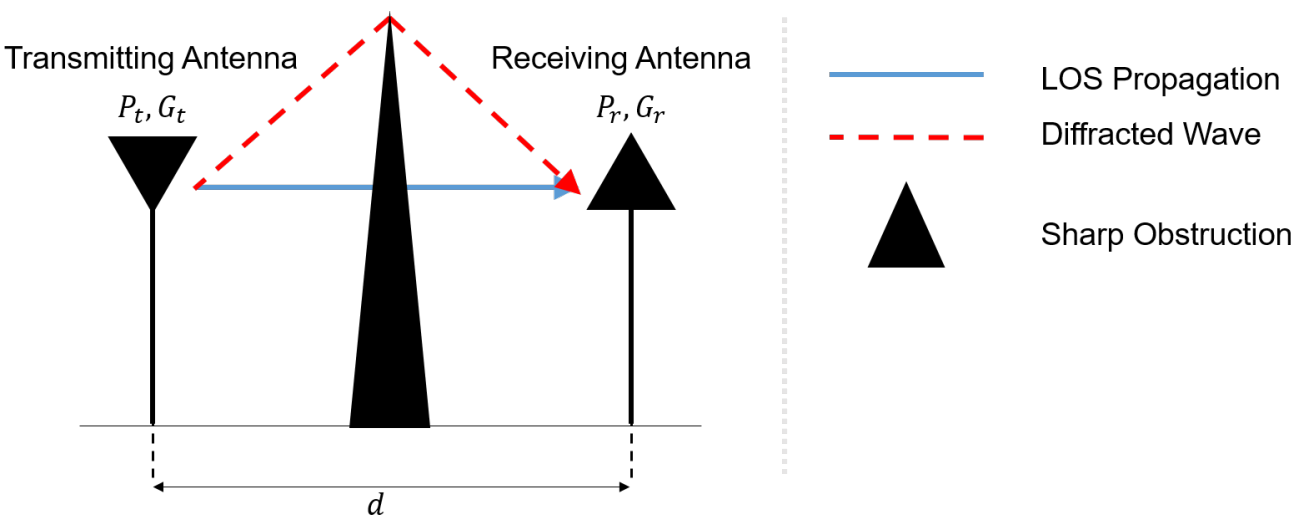

Figure 3.7: Fresnel Knife Edge Diffraction Model Parameters

As can be seen in Figure 3.7, the diffracted waves bend around the obstructive object and arrive to the receiving antenna with phase delay due to longer propagation path than the LOS propagation. This longer path results in phase shift:

$$
\phi=\frac{2 \pi\left(d+d^{\prime}\right)}{\lambda}
$$

This phase shift can be characterized as a relative to LOS propagation path:

$$
\Delta \phi=\frac{2 \pi \frac{h^{2}\left(d+d^{\prime}\right)}{2 d d^{\prime}}}{\lambda}=\frac{\pi}{2} v^{2}
$$

where $v=h \sqrt{\frac{2\left(d+d^{\prime}\right)}{\lambda d d^{\prime}}}$ is called Fresnel-Kirchoff diffraction parameter. With the help of Fresnel-Kirchoff diffraction parameter, the path loss occurring due to the diffraction can be empirically characterized with the diffraction parameter as below: 


$$
L(v)= \begin{cases}20 \log _{10}(0.5-0.62 v), & -0.8 \leq v<0 \\ 20 \log _{10}\left(0.5 e^{-0.95 v}\right), & 0 \leq v<1 \\ 20 \log _{10}\left(0.4-\sqrt{0.1184-(0.38-0.1 v)^{2}}\right), & 1 \leq<v<2.4 \\ 20 \log _{10}\left(\frac{0.225}{v}\right), & v>2.4\end{cases}
$$

\subsubsection{Scattering}

As mentioned earlier, N-LOS propagation mechanisms play a significant role in the characterization of the radio wave propagation. Therefore, these propagation mechanisms are important in radiolocation literature as important as to wave propagation. Scattering is a N-LOS propagation mechanism where radio waves are disturbed by an attenuator whose dimensions are comparable to the wavelength of the radio wave and diffused into different propagation paths. For instance, rain drops seem to scatter radio waves whose wavelength is approximately $10 \mathrm{GHz}(\lambda=3 \mathrm{~cm})$ due to comparable dimensions of the raindrops and the wavelength. Since the direction of the new wave-fronts created by the scattering mechanism, the effect of scattering mechanism is often formulated in empirical manner.

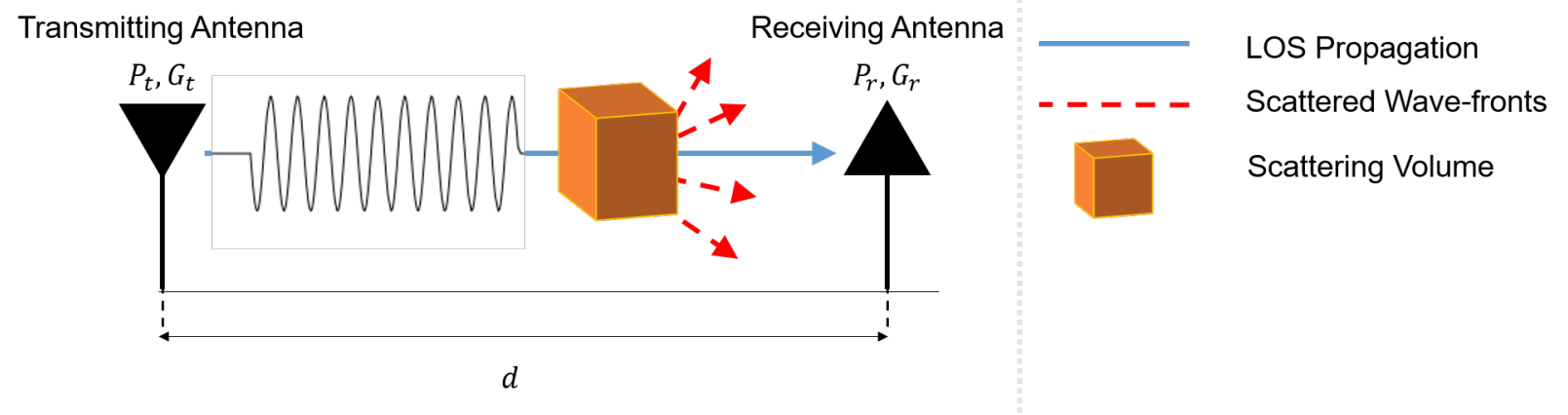

Figure 3.8: Scattering mechanism diffuse the radio waves, resulting in new wave-fronts directed at different angles 


\subsection{Log-distance Path Loss Model}

LDPL is an indoor wave propagation model which formulates the correlation between the transmitted and received signal strength as a function of Euclidean distance between an access point and the agent.

Let $P L(d)$ be the difference between transmitted signal strength and RSS fingerprint obtained at a test point $d$ distance away from the access point. This difference is commonly referred as path loss. LDPL model, formulates the path loss of the test point relative to a reference point $d_{0}$ distance away from the access point:

$$
P L(d)=\overline{P L}\left(d_{0}\right)+10 n \log _{10} \frac{d}{d_{0}}+X_{\sigma}
$$

where $\overline{P L}\left(d_{0}\right)$ and $X_{\sigma}$ are the mean path loss measured at the reference point, a normal distribution with zero mean and $\sigma$ standard deviation, respectively. The second term in the model denotes the relative path loss as a function of $\frac{d}{d_{0}}$ and path loss exponent $n$. Table 3.2 shows different values of path loss exponent $n$ depending on the environment and communication path.

As can be seen in the formulation of the LDPL model, wave propagation not only depends on separation distance $d$, but also path loss exponent $n$ and standard deviation $\sigma$. Therefore, these parameters should be estimated from the collected data. A least squares approach can be used to obtain the model parameters:

$$
n^{*}, \sigma^{*}=\underset{n, \sigma}{\arg \min } \frac{1}{n_{s}} \sum_{i=1}^{n_{s}}\|P L(d)-\widehat{P L}(d)\|_{2}^{2}
$$

where $\widehat{P L}(d)$ is the estimated path loss occurred at the distance $\mathrm{d}$ with path loss $n$ and standard deviation $\sigma$. 


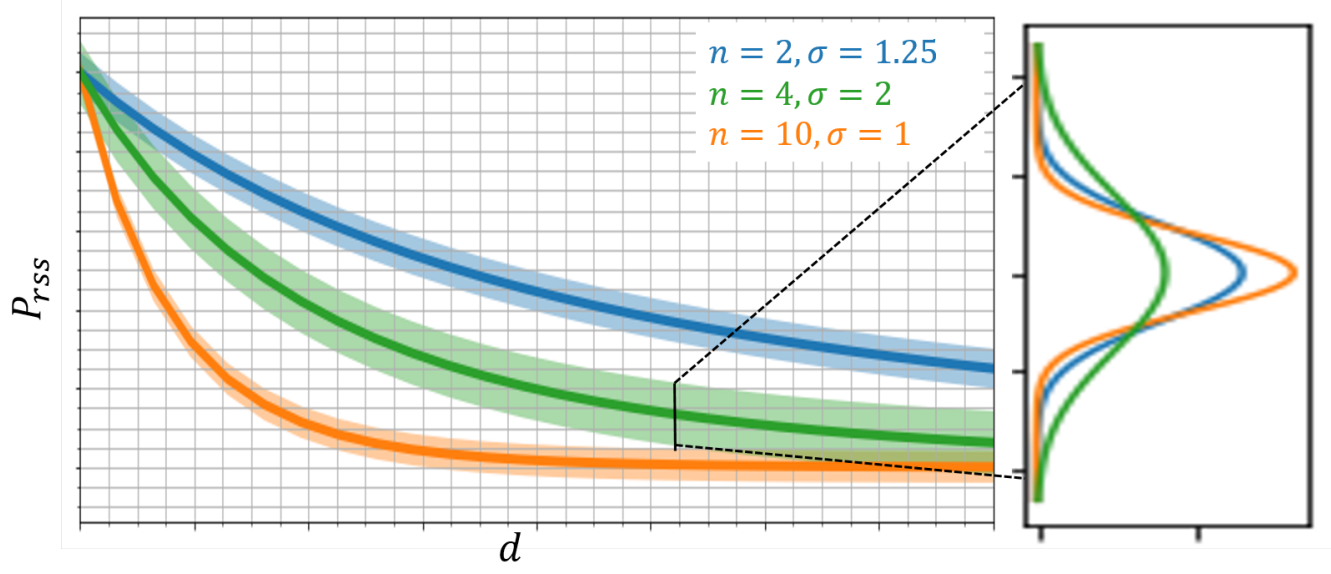

Figure 3.9: LDPL Model Parameters and Path Loss: As can be seen in the figure, path loss exponent $n$ governs how fast the received signal decays, while $\sigma$ models the shadowing factor in the measurements.

LDPL formulation can be solved for the Euclidean distance $d$, for each measured path loss $P L(d)$ at an unknown location, and estimated model parameters $\left[n^{*}, \sigma^{*}\right]^{T}$. Consequently, each fingerprint collected from access points within the agent's radio coverage will induce a circle in $\mathbb{R}^{2}$ centered at the access point. The agent location can be then determined with trilateration applied on the induced circles.

Table 3.2: Various values of path loss exponent based on the environment and the propagation path.

\begin{tabular}{ll}
\hline$<2$ & the space structure guides the radio waves along the propagation path \\
\hline$=2$ & empty space overall propagation path is dominated LOS communication \\
\hline$>2$ & $\begin{array}{l}\text { There are obstructions along the propagation path multipath effect takes places due } \\
\text { to N-LOS communication }\end{array}$ \\
\hline
\end{tabular}




\subsection{Indoor Radiolocation with Trilateration}

The indoor radiolocation problem of interest in this paper is to locate an agent equipped with a radio module in an indoor environment that installs some number of access points in a distributed manner. Let indoor environment and some number of access points with known poses in the environment be $\Omega$ and $m_{1: k}$, respectively. At an unknown location, the agent collects $n_{m}$ number RSS fingerprints from all the access points within its field of coverage. The distances between the agent and the access points can be inferred with the use of RSS fingerprints and the radio wave propagation model. Consequently, the global position of the agent can be then obtained by using the trilateration method.

Let the measured distance between the agent and $i^{\text {th }}$ access point which resides at $\vec{x}^{i}=\left[x^{i}, y^{i}, z^{i}\right]^{T}$ be $d_{i}$. Then the position of the agent can be represented as:

$$
d_{i}^{2}=\left(x^{t}-x^{i}\right)^{2}+\left(y^{t}-y^{i}\right)^{2}+\left(z^{t}-z^{i}\right)^{2}
$$

As can be seen in the Equation (3.15), each equation induces a sphere centered at the access point with $d_{i}$ radius. Since all the access points in the field of coverage of the agent lead to an equation, simultaneous solution of these equation yields to the position of the agent.

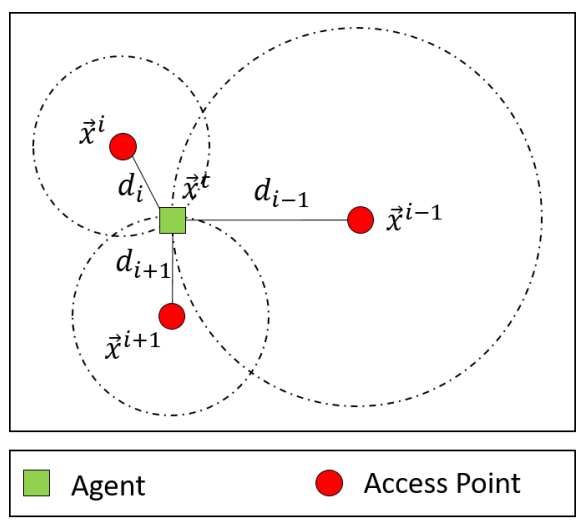

Figure 3.10: Trilateration of an agent with separation distance estimations 
By using $j^{\text {th }}$ equation as a linearization pivot, all the quadratic unknown terms can be eliminated in Equation (3.15). Therefore, the set of equations attained can be represented in a matrix form. The linear system can be then solved for $\vec{x}=\left[\begin{array}{ll}\left(x^{t}-x^{j}\right), & \left(y^{t}-y^{j}\right), \quad\left(z^{t}-z^{j}\right)\end{array}\right]^{T}$ to determine the position vector of the agent.

$$
\begin{gathered}
\boldsymbol{A}=\left[\begin{array}{ccc}
x^{1}-x^{j} & y^{1}-y^{j} & z^{1}-z^{j} \\
x^{2}-x^{j} & y^{2}-y^{j} & z^{2}-z^{j} \\
\vdots & \vdots & \vdots \\
x^{j-1}-x^{j} & y^{j-1}-y^{j} & z^{j-1}-z^{j} \\
x^{j+1}-x^{j} & y^{j+1}-y^{j} & z^{j-1}-z^{j} \\
\vdots & \vdots & \vdots \\
x^{n_{m}}-x^{j} & y^{n_{m}}-y^{j} & z^{n_{m}}-z^{j}
\end{array}\right] \\
\vec{b}=0.5\left[\begin{array}{c}
d_{j}^{2}-d_{1}^{2}+d_{1 j}^{2} \\
d_{j}^{2}-d_{2}^{2}+d_{2 j}^{2} \\
\vdots \\
d_{j}^{2}-d_{j-1}^{2}+d_{j-1 j}^{2} \\
d_{j+1}^{2}-d_{j+1}^{2}+d_{j+1 j}^{2} \\
\vdots \\
d_{j}^{2}-d_{n_{m}}^{2}+d_{n_{m}, j}^{2}
\end{array}\right]
\end{gathered}
$$

where $d_{i j}$ represents the distance between $i^{t h}$ and $j^{\text {th }}$ markers. Due to sensor and process uncertainty, direct solution of trilateration often yields to unwanted solutions [48]. Therefore, the least squares approach is often used in trilateration problems to cope with noisy measurements. The least square formulation minimizes overall localization error $S$, which is induced by the measurement noise. 


$$
\begin{aligned}
& S=\vec{r}^{T} \vec{r}=(\vec{b}-\boldsymbol{A} \vec{x})^{T}(\vec{b}-\boldsymbol{A} \vec{x}) \\
& \overrightarrow{\hat{x}}=\underset{x}{\arg \min } S
\end{aligned}
$$

If the $\boldsymbol{A}^{T} \boldsymbol{A}$ is not singular, the solution for Equation (3.16) can be derived as Equation (3.17).

$$
\overrightarrow{\hat{x}}=\left(\boldsymbol{A}^{T} \boldsymbol{A}\right)^{-1} \boldsymbol{A}^{T} \vec{b}
$$

\subsection{Summary}

This section has provided a general framework for radio wave propagation mechanisms and models and their use in radiolocation techniques. In order to provide a complete framework, first the fundamentals of indoor radio wave propagation mechanisms has been laid out where it is often observed that multi-path effect takes place due to obstructions in the environments. These mechanism are useful in ray-tracing methods where received signal is decomposed such that the exact propagation path between transmitting and receiving antennae is estimated.

In the context of indoor radiolocation techniques, ray-tracing methods are often results in inaccurate estimation of the propagation path due to dynamic nature of the obstructions in the environment. Therefore, in radiolocation techniques, received signal is decomposed such that the detrimental effects of the multi-path effect, which results many copies of the transmitted signal with different phase delay, is characterized as N-LOS attenuation posed by the environment. Therefore, the overall accuracy of the radiolocation techniques directly attributes to accuracy of the propagation model and this ability of characterization of diverse propagation mechanisms employed in the radiolocation technique plays a significant 
role. From this observation, a radio wave propagation model which can characterize the attenuation caused by the environment is still needed to improve the accuracy of the current indoor radiolocation techniques. 


\section{Chapter 4}

\section{Grid Based Indoor Radiolocation Technique Based on SCPL}

This chapter presents a grid cell based radiolocation technique based on Spatially Coherent Path Loss (SCPL) model. With the objective stated in Chapter 1 in mind, the proposed path loss model presented in this chapter would be able to characterize the radio wave propagation even in the presence of the obstacles. Based on the proposed propagation model, a grid cell based radiolocation technique which employs SCPL in its core to localize an agent in an indoor environment.

The radiolocation problem of interest to locate an agent equipped with a radio transceiver module in an indoor environment. Once the agent reaches to a new location, it interrogates all the accessible access points placed in the environment. The interrogation results, i.e. the responses of the access points, are used to capture RSS fingerprints. With known transmission power for each access point, the power loss occurring along the propagation path is calculated. The magnitude of attenuation is then used to localize the agent in the environment. Figure 4.1 demonstrates the problem of consideration in greater details. 


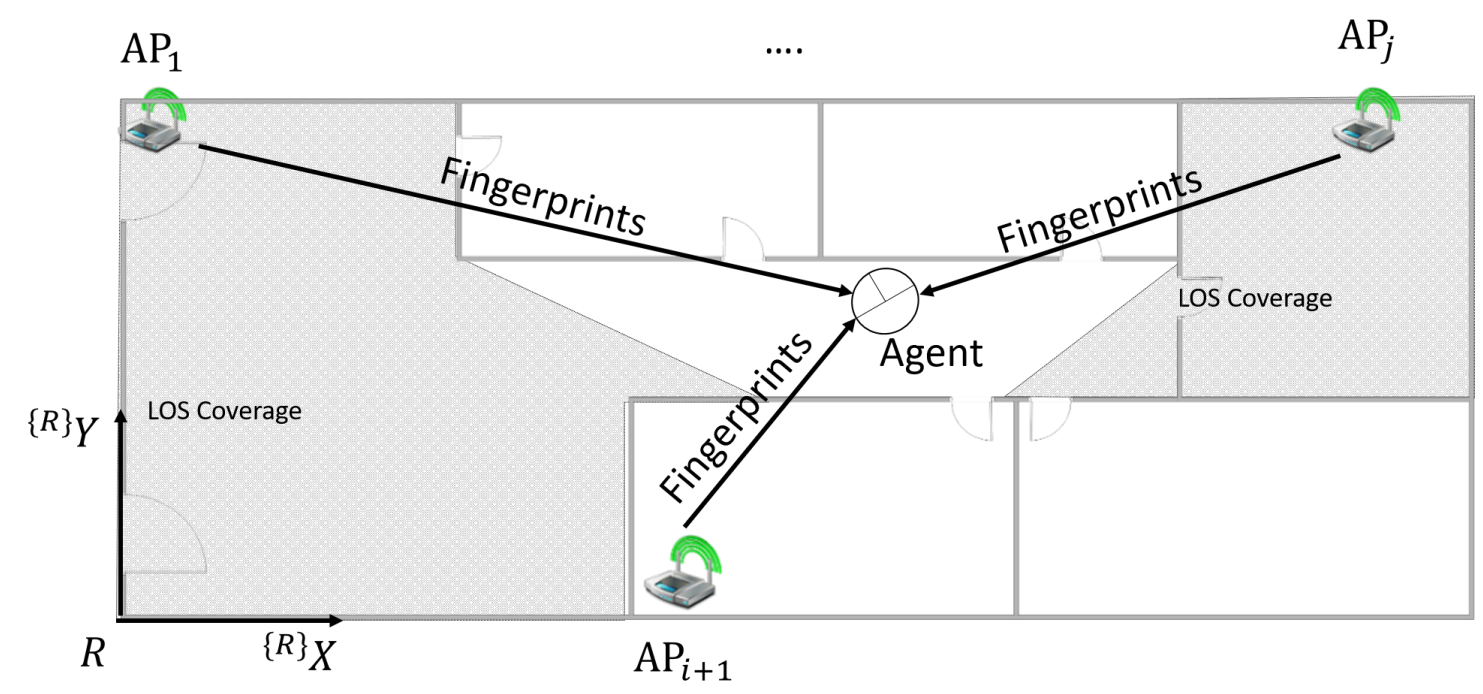

Figure 4.1: Indoor radiolocation of interest: An agent at an unknown location in the indoor environment interrogates the access points whose positions to capture RSS fingerprints. The fingerprint are then used to choose the parameters of the SCPL model. By using the RSS fingerprints and the estimated model parameters, the attenuation profile of the region where the agent is location is calculated. Consequently this information is employed to estimate the separation distance between the agent and each access points.

Figure 4.2 demonstrates the schematic diagram of the proposed grid-based indoor radiolocation technique. Given the RSS fingerprint obtained at an unknown location, the SCPL model parameters are first chosen with a feed-forward neural network. By using the parameters chosen by the neural network, SCPL is then used with the RSS fingerprint to estimate the distances between the access points and the agents. Least-squares trilateration technique is successively applied on the attained distances to determine the location of the agent. Consequently, the result of the trilateration is represented with a probabilistic distribution over the indoor localization environment $\Omega$. This section covers the details of each component of the localization algorithm in further detail. 


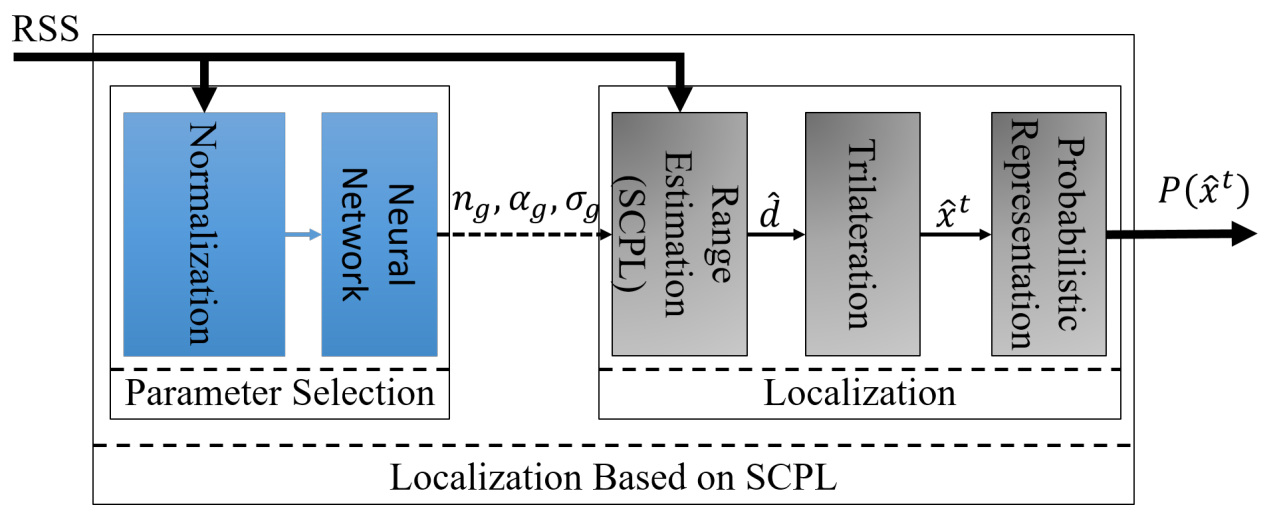

Figure 4.2: Schematic diagram of the proposed localization algorithm: The model parameters are first chosen with a feed-forward neural network. The distance between the target and the access points is attained with SCPL. Consequently, trilateration is used to determine the position of the agent which results in a belief function over the localization environment $\Omega$.

\subsection{Spatially Coherent Path Loss Model}

Let $\Omega=\left\{g(i, j) \mid i=1 \ldots n_{g x}, j=1 \ldots n_{g y}\right\}$ be the localization environment which is uniformly divided $n_{g x}$ by $n_{g y}$ number of grid-cells. $g(i, j)$ represents the grid-cell resides in $[i, j]$ in grid-coordinates. Figure 5.1 demonstrates the grid-cell structure of the environment in detail. SCPL model characterizes the radio wave propagation for each grid-cell by evaluating the formulation below:

$$
P L(d)=P L_{\text {los }}(d)+P L_{\text {att }}(d)+X_{\sigma}
$$

where $d$ is the Euclidean distance between an access point and the grid cell center $c(i, j)$.

$$
d_{i}=\left\|\vec{x}^{i}-c(i, j)\right\|_{2}
$$

SCPL model characterizes the measured path loss acquired at a test point with $d$ distance away from the access point as the sum of the LOS propagation path loss $P L_{l o s}(d)$ and an explicit attenuation term $P L_{\text {att }}(d) . P L_{l o s}(d)$ is defined with FFSE [46] whose details 


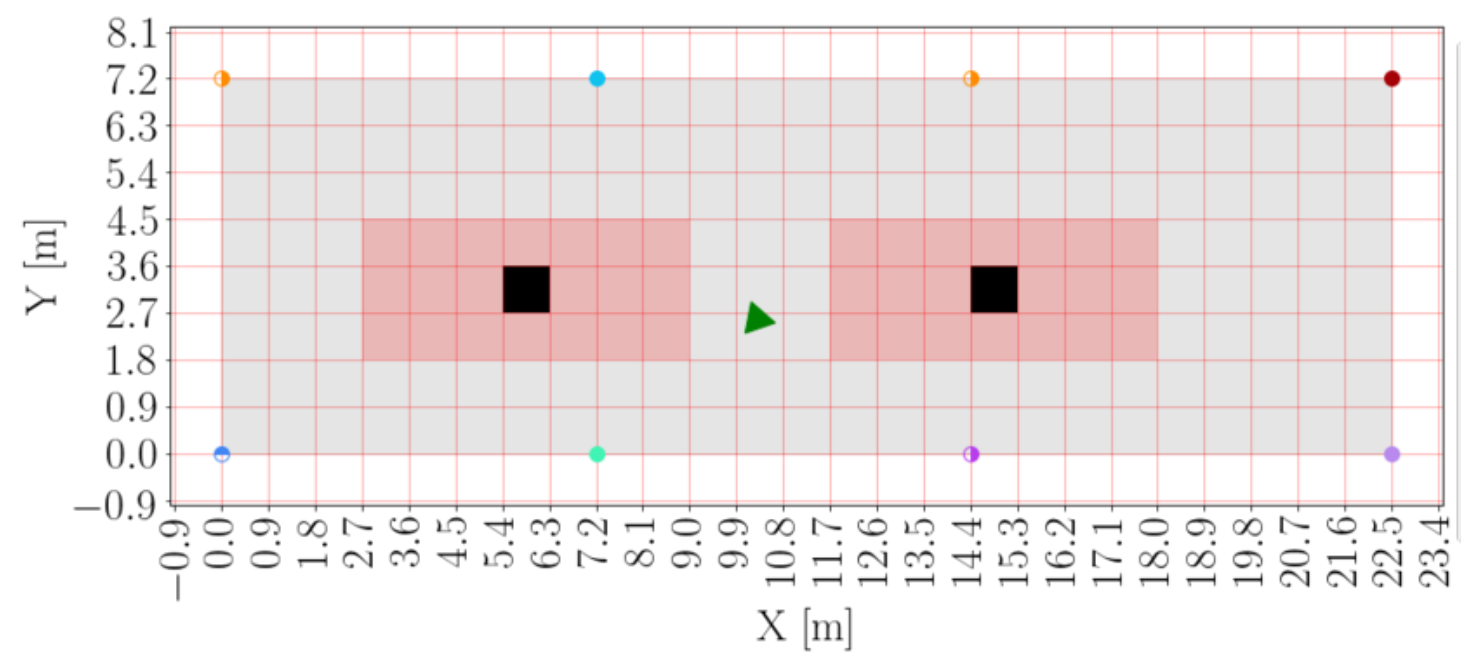

Figure 4.3: The green triangle represents the location of the agent. The shaded gray area divided into grid cells represents the localization environment $\Omega$. The circles around the environment represent the access point locations, while red region in the environment represents the region resulting in multipath effect.

can be found in Section 3.2.1. On the other hand, $P L_{a t t}(d)$ the attenuation caused by the obstacles in grid-cell $g$ is formulated as given in Equation (4.3).

$$
P L_{\text {att }}(d)=10 n_{g} \log _{10} \alpha_{g} d
$$

where $n_{g}$ and $\alpha_{g}$ represent the path loss exponent and distance correction factor for grid-cell $g$. Following section provides further details of the model parameters.

\subsubsection{Model Parameters}

SCPL model characterizes the attenuation of the environment in each grid cell by using three parameters. Table 4.1 provides each model parameters and their corresponding descriptions. 


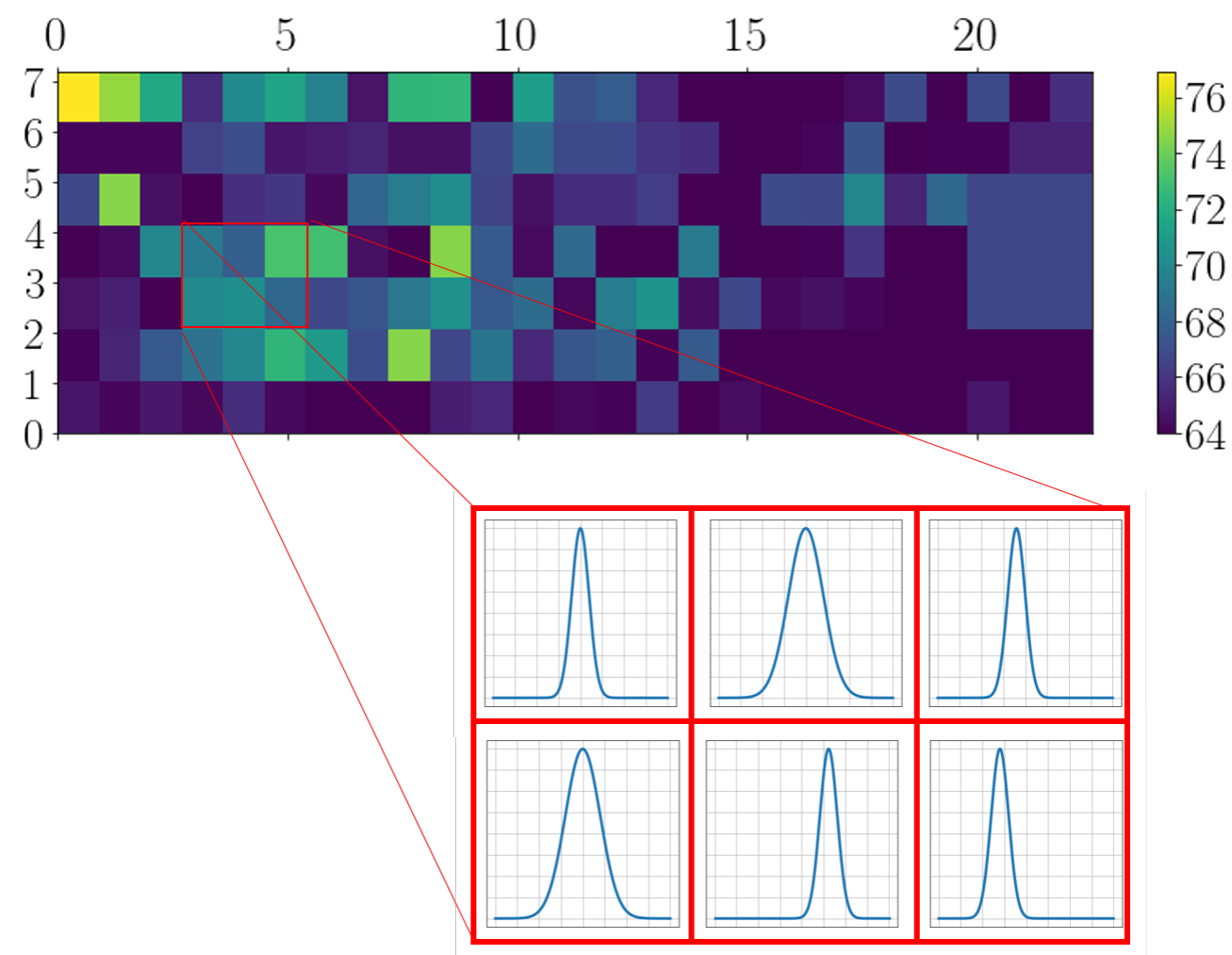

Figure 4.4: This figure demonstrates how SCPL characterizes the propagation in an environment. The colors in the grid cells represent the mean of the measured path loss for each grid cell, while zoomed window shows the probability distribution function of measured path loss within those grids marked on the environment.

Table 4.1: The descriptions of SCPL model parameters.

$n_{g} \quad$ The path loss exponent estimated for grid-cell $g$. If the grid-cell $g$ is closer to an obstruction, it is expected to see higher values in order to model the attenuation better.

$\alpha_{g} \quad$ Distance correction factor. Since the N-LOS propagation path should be longer than LOS path, the length of the propagation path is corrected with this parameter.

$\sigma_{g} \quad$ The estimated deviation of the normal distribution which is modeling the slow fading of the channel.

\section{Path Loss Exponent $n_{g}$}

Path loss exponent $n_{g}$ represents the path loss exponent for grid cell $g$ and governs the attenuation magnitude occurring the grid cell. As the exponent increases, the expected 
attenuation increases. Figure 4.5 shows the simulated effect of an increasing $n_{g}$ for an arbitrary grid cell with fixed parameters except the path loss exponent. As can be seen in the figure, increasing attenuation of the radio waves can be modeled with increased values of path loss exponent. This correlation implicitly defines where in the environment N-LOS propagation mechanisms taking place.

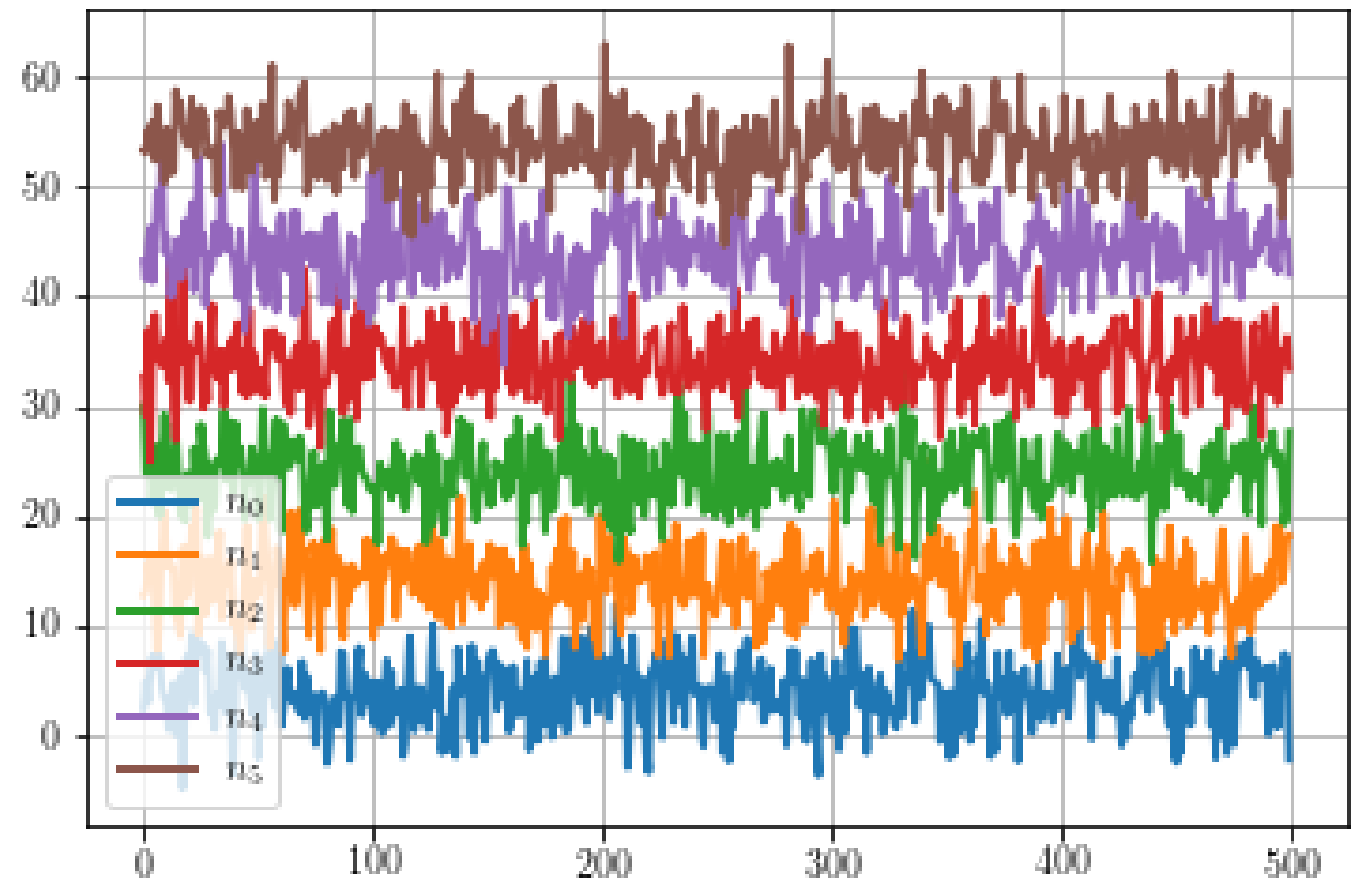

Figure 4.5: Effect of increasing: $n_{0}<n_{1}<n_{2}<n_{3}<n_{4}<5$. As can be seen in the figure, the increase in the path loss measurements for each grid cell results in increase in the path loss exponent $n_{g}$.

\section{Distance Correction Factor $\alpha_{g}$}

LDPL model represents the attenuation of the environment as a relative term to the reference point as a function of the Euclidean distance between the agent and the access point. 
However, this formulation yields inaccurate results in the cases where the propagation path contains obstacles. Since N-LOS propagation mechanisms such as reflection, diffraction and scattering, is more likely to be observed in such cases, the real propagation paths differ from the Euclidean distance between the agent and the access points. In small environments, this difference might be negligible; however, in large environments such as warehouses, manufacturing sites, this difference adds up to a significant deviation from the actual propagation path; hence the path loss. Figure 4.6 represents a similar case where two different N-LOS propagation paths are compared. As the distance between the access point and the agent is small, the difference between the actual propagation path and the direct path is negligible. On the other hand, once the agent interrogates another access point with greater separation distance, this difference results in higher errors in localization. By considering this fact, a distance correction factor $\alpha_{g}$ for each grid cell is introduced. Since the N-LOS propagation path will be longer than LOS path, $\alpha_{g}$ estimates the N-LOS distance, while $n_{g}$ serves as a path loss exponent.

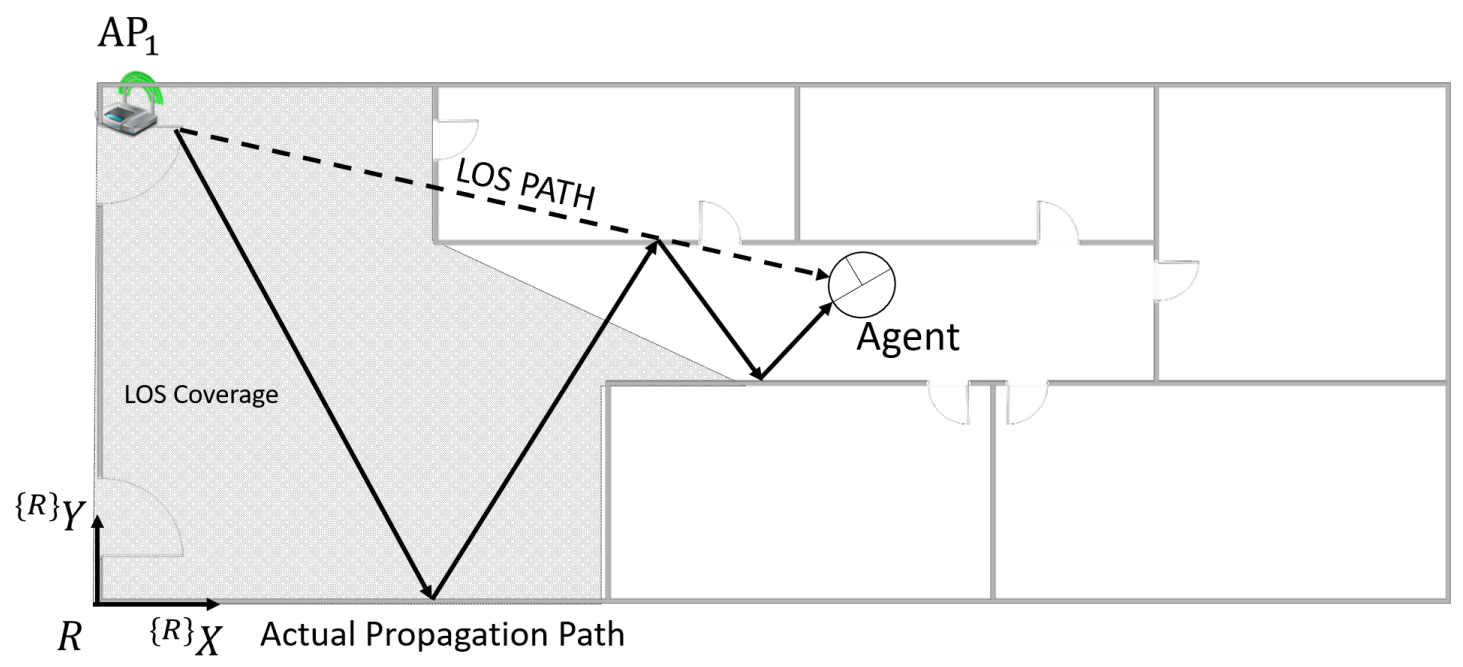

Figure 4.6: Since the N-LOS propagation path will be longer than LOS path, the actual propagation path is estimated with a multiplicate term $\alpha_{g}$. 


\section{Fingerprint Variation $X_{\sigma_{g}}$}

RSS fingerprints display variation in magnitude even if the agent is stationary in the environment. In order to account for this variation in the model, a random variable is employed in the formulation. Random variable $X_{\sigma_{g}}$ models the variation of the fingerprint acquired in the same grid cell with a normal distribution distributed with a zero mean, and $\sigma_{g}$ standard deviation.
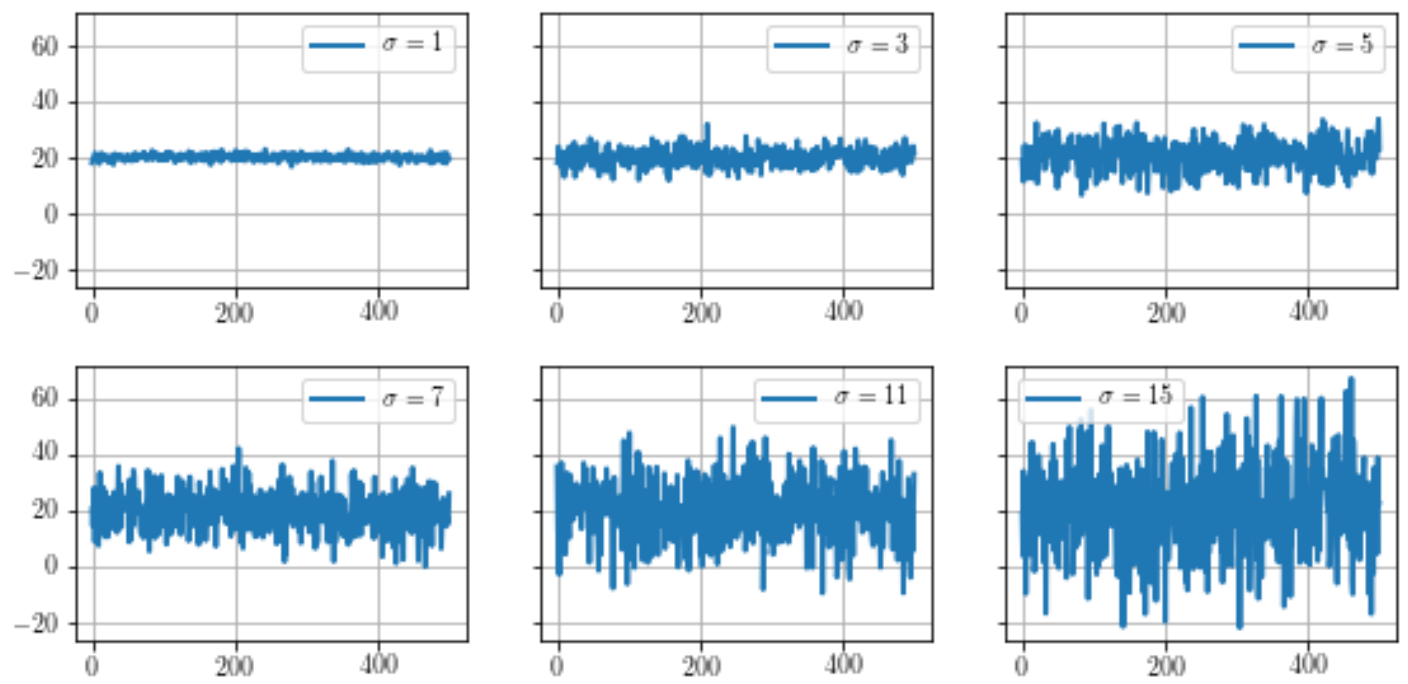

Figure 4.7: Effect of increasing $\sigma_{g}$.

\subsubsection{Parameter Estimation for SCPL Model}

Similar to attenuation term in LDPL, SCPL models the attenuation as a logarithmic function. However, the main difference is that SCPL does not model the attenuation relative to the reference point. Rather, the path loss exponent $n_{g}$, the distance correction factor $\alpha_{g}$ and $X_{\sigma_{g}}$ is a function of the position the agent. For each grid-cell, model parameters are estimated; hence, the propagation of the radio waves are identified locally. As a result of this, for each grid-cell, the irregularities of the environment can be modeled locally. 
SCPL model parameters are estimated with a least-squares approach which minimize the path loss estimation error in grid-cell $g$. Once these parameters are estimated from the collected fingerprints, the proposed path loss model can be used to estimate the separation distance from all of the access points. The formulation for the estimation is given in Equation (4.4).

$$
n^{*}(i, j), \alpha^{*}(i, j), \sigma^{*}(i, j)=\underset{n, \alpha, \sigma}{\arg \min } \frac{1}{n_{s} n_{x} n_{y}} \sum_{v=j-\frac{n_{y}}{2}}^{j+\frac{n_{y}}{2}} \sum_{u=i-\frac{n_{x}}{2}}^{i+\frac{n_{x}}{2}} \sum_{s=1}^{n_{s}}\|P L(u, v)-\widehat{P L}(i, j)\|_{2}^{2}
$$

where $P L(u, v)$ represents the path loss measured in grid-cell $g(u, v)$, while $n_{s}$ represent the number of fingerprints used in the estimation.

As can be seen in Equation (4.4) and Figure 4.8, for each grid-cell $g(i, j)$, the loss function considers neighboring grid-cells with spread factor of $n_{x}$ and $n_{y}$. Assuming grid-cells residing in a local vicinity should show similar propagation characteristics, the parameters of grid-cell $g(i, j)$ are attained by employing the fingerprints collected in neighboring grid-cells as well. By incorporating the neighboring grid-cells, the data points needed to estimate the model parameters are decreased, as well as the locality coherence is ensured.

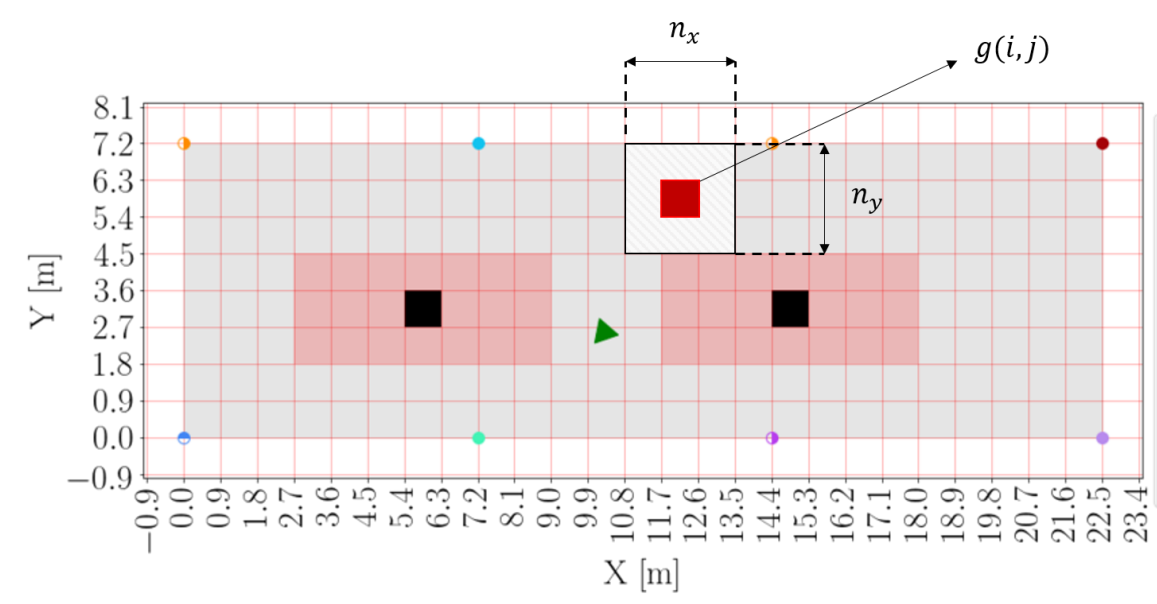

Figure 4.8: Parameter Estimation for SCPL Model 
Murat Ambarkutuk Chapter 4. Radiolocation based on Spatially Coherent Path Loss (SCPL) Model 54

\subsection{Grid-based Radiolocation Based on SCPL Model}

Figure 4.2 demonstrates the schematic diagram of the proposed localization technique. Given a fingerprint $P_{r}(i, j)$ acquired in an unknown grid-cell $g(i, j)$, the neural network is first employed to choose a set of SCPL model parameters. After selecting a set of model parameters, the acquired fingerprint $P_{r}(i, j)$ is used to estimate the distances between the access points and the target. The estimated distance between the access points and the agent is utilized in a least-square trilateration framework such that the estimation for each access point can be fused and the agent location can be determined. Since SCPL model results in a distribution for each distance estimation, the trilateration also yields a distribution representing the position of the agent.

\subsubsection{Parameter Learning and Parameter Selection}

Given a fingerprint $P_{r}(i, j)$ acquired in an unknown grid-cell $g(i, j)$, the neural network is first employed to choose a set of SCPL model parameters. Let $\vec{p}(i, j)=[n(i, j), \alpha(i, j), \sigma(i, j)]^{T} \in$ $\mathbb{R}^{3}$ are first chosen from all the estimated parameters $\boldsymbol{P} \in \mathbb{R}^{n_{g x} \times n_{g_{y} \times 3}}$. This parameter selection is achieved with a 3-layer feedforward neural network where each layer of the network can be represented as Equation (4.5). The structure of the neural network can be seen in Figure 4.9.

$$
f_{n}\left(\vec{h}_{n}\right)=\sigma\left(\boldsymbol{W}_{n} \vec{h}_{n}+\vec{b}_{n}\right)
$$

where $\boldsymbol{W}_{n}$ and $\vec{b}_{n}$ represent the learned weight matrix and bias vector of $n^{\text {th }}$ layer, respectively. $\sigma(\cdot)$ denotes the nonlinearity function, while $\vec{h}_{n}$ is the input of the $n^{\text {th }}$ hidden layer.

Since all the model parameters are positives values, ReLu [49] is used as the nonlinearity 


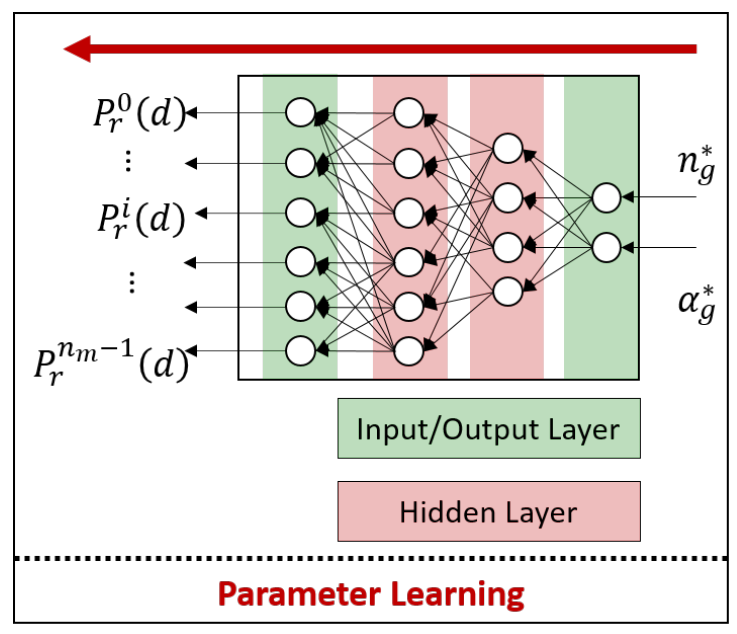

(a) Parameter Learning Phase of the Feedforward Neural Network: In this phase, the weight matrices and bias vectors of each layer is learned with labeled data.

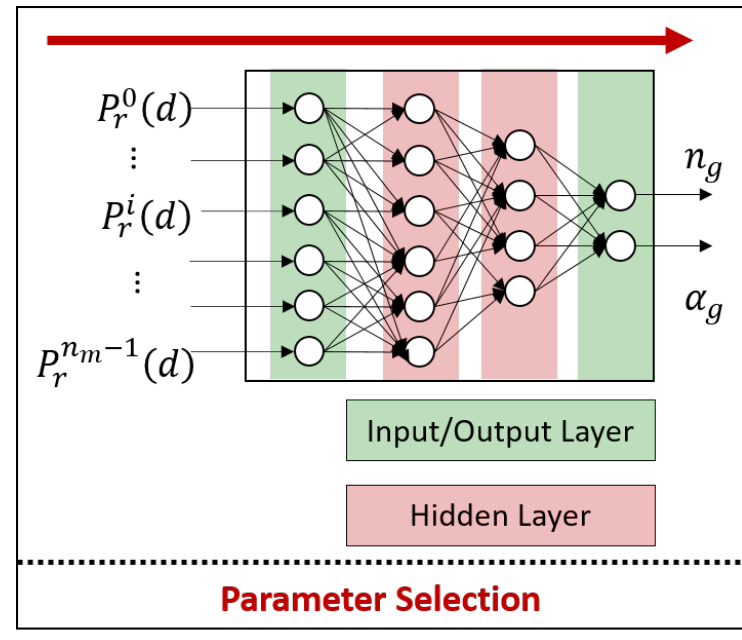

(b) Parameter Learning Phase of the Feedforward Neural Network: In this phase, the weight matrices and bias vectors of each layer is learned with labeled data.

Figure 4.9: Left: Parameter Learning Phase where RSS measurements are optimal model parameters are used to train the neural network. This phase is conducted once before deploying during the offline phase.

Right: Parameter Selection Phase where new RSS measurements are used with the trained neural network to estimate model parameters

function in SCPL model. Equation (4.6) represents the ReLu function.

$$
\sigma\left(\vec{h}_{n}\right)=\max \left(0, \vec{h}_{n}\right)
$$

Before training the neural network the input data, the measurements, is normalized such that the measurements of the each access points centered at zero with a variance of 1 .

Consequently, the model parameters are inferred by utilizing weights and biases of each layer consecutively, followed by the nonlinearity function. This parameter selection phase forms the first step of the online stage of the proposed radiolocation technique. 
Murat Ambarkutuk Chapter 4. Radiolocation based on Spatially Coherent Path Loss (SCPL) Model 56

\subsubsection{Separation Distance Estimation and Target Localization}

After model parameters are chosen, the distance from the access point and to the agent is be estimated by utilizing the SCPL model. As stated earlier, SCPL model can be represented with a closed form formulation:

$$
P L(d)=P L_{\text {los }}(d)+P L_{\text {att }}(d)+X_{\sigma}
$$

As shown in Equation (4.7), SCPL models the path loss of each grid cell with a normal

distribution. Please note $X_{\sigma} \sim \mathcal{N}\left(0, \sigma_{g}^{2}\right)$. Therefore, separation distance estimations can be represented within the probabilistic framework. Let $P_{r}^{i}(d)$ is a received RSS fingerprint from access point $i$, then probability distribution representing the distance between the agent and $i^{t h}$ access point can be represented as:

$$
\begin{aligned}
P L(d) & =P L_{\text {los }}(d)+P L_{\text {att }}(d)+X_{\sigma} \\
P L(d) & =\frac{P_{t} G_{t} G_{r} \lambda^{2}}{(4 \pi d)^{2}}+10 n_{g} \log _{10} \alpha_{g} d+X_{\sigma} \\
\Longrightarrow \log _{10}\left(D_{i}\right) & \sim \mathcal{N}\left(\mu, s^{2}\right)
\end{aligned}
$$

where

$$
\begin{aligned}
\mu & =\frac{K+P_{t}-P_{r}^{i}(d)}{20+n_{g}} \\
s & =\frac{\sigma_{g}}{20+n_{g}} \\
K & =10 \log _{10}\left(G_{t} G_{r}\right)+20 \log _{10}(\lambda)-20 \log _{10}(4 \pi)-n_{g} \log _{10}\left(\alpha_{g}\right)
\end{aligned}
$$

As can be seen in Equation (4.8), SCPL results in lognormal distribution for separation 
distance estimations. This resulting random variable can be seen in Figure 4.10.

By using the properties of a lognormal distribution, the estimated distance has a mean of:

$$
\begin{aligned}
\hat{d}_{i} & =\mathbb{E}\left[D_{i}\right] \\
& =\exp \left(\mu+0.5 s^{2}\right) \\
\hat{d}_{i} & =\frac{K+P_{t}-P_{r}^{i}(d)}{20+n_{g}}+0.5 \frac{\sigma_{g}}{20+n_{g}}
\end{aligned}
$$

The variance of the estimated distance can be stated as:

$$
\begin{aligned}
\operatorname{Var}\left(D_{i}\right) & =\mathbb{E}\left[\left(D_{i}-\mathbb{E}\left[D_{i}\right]\right)^{2}\right] \\
& =\exp \left(2\left(\mu+s^{2}\right)\right)-\exp \left(2 \mu+s^{2}\right) \\
\operatorname{Var}\left(D_{i}\right) & =\exp \left(2\left(\frac{K+P_{t}-P_{r}^{i}(d)}{20+n_{g}}+\left(\frac{\sigma_{g}}{20+n_{g}}\right)^{2}\right)\right)-\exp \left(2 \frac{K+P_{t}-P_{r}^{i}(d)}{20+n_{g}}+\left(\frac{\sigma_{g}}{20+n_{g}}\right)^{2}\right)
\end{aligned}
$$

In order to localize the agent the global position of the agent in the environment, the distance estimations for each access point should be incorporated. In the light of this, a least-squares trilateration method is employed in order to determine the location of the target.

Let $P(i, j)$ be the probability of the target residing in $g(i, j)$, resulted from the leastsquares trilateration. Then $\mathbb{P}(\Omega)$ represents the probability distribution function over all the grid-cells.

$$
\mathbb{P}(g(i, j))=P\left(\vec{x}^{t}=c(i, j)\right)
$$




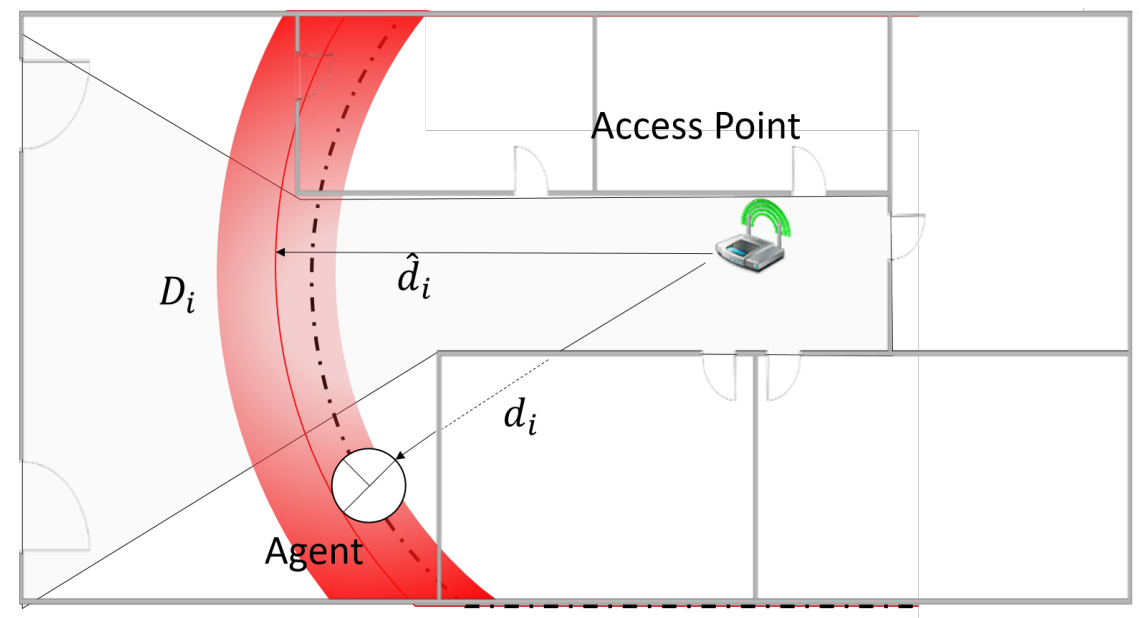

Figure 4.10: Separation distance estimation with SCPL: $D_{i}$ represents the resulting random variable for separation distance between the agent and the $i^{t h}$ access point. $\hat{d}_{i}$ and $d_{i}$ represent the mean of the random variable and the actual distance between the agent and the access point, respectively.

where $c(i, j)$ represents the center of grid cell $g(i, j)$.

The quantification of the probability distribution function $\mathbb{P}(\Omega)$ is then achieved with expectation operator. The final localization result is represented as below:

$$
\overrightarrow{\hat{x}}^{t}=\mathbb{E}[\mathbb{P}(\Omega)]
$$

By representing the localization as a distribution over $\Omega$, the model can better account for error resulted from model inaccuracies, environment irregularities and trilateration.

\subsection{Summary}

This chapter presented the proposed grid based radiolocation technique. First, SCPL model was introduced. SCPL model characterizes the radio wave propagation in an environment 
by profiling the attenuation of the radio waves. This characterization is handled in a grid cell based manner such that the attenuation profile can have different values depending on where the agent is. This level of granularity in attenuation profile, is then employed in a radiolocation technique with which an agent in an environment can be located with radio waves. With the use of higher level granularity in attenuation profile and probabilistic representation of the localization over the grid cells, the proposed technique can potentially handle the effect of irregular attenuation sources, and the noise in the fingerprint measurements. 


\section{Chapter 5}

\section{Experiments and Results}

This section will outline the details of the experimentation conducted. The structure of the environment is first described as well the determination of the grid-cell size, followed by the details of the fingerprint collection process. Finally, evaluation of the parameter estimation, parameter selection and the overall SCPL model performance is covered.

\subsection{Experimental Setup}

\subsubsection{Localization Environment}

In this section, the properties and characteristics of the environment in which the experimentations were conducted. The structure of the environment is first provided, followed by the determination of grid cell size. Finally, the details of the dataset structure is provided. 


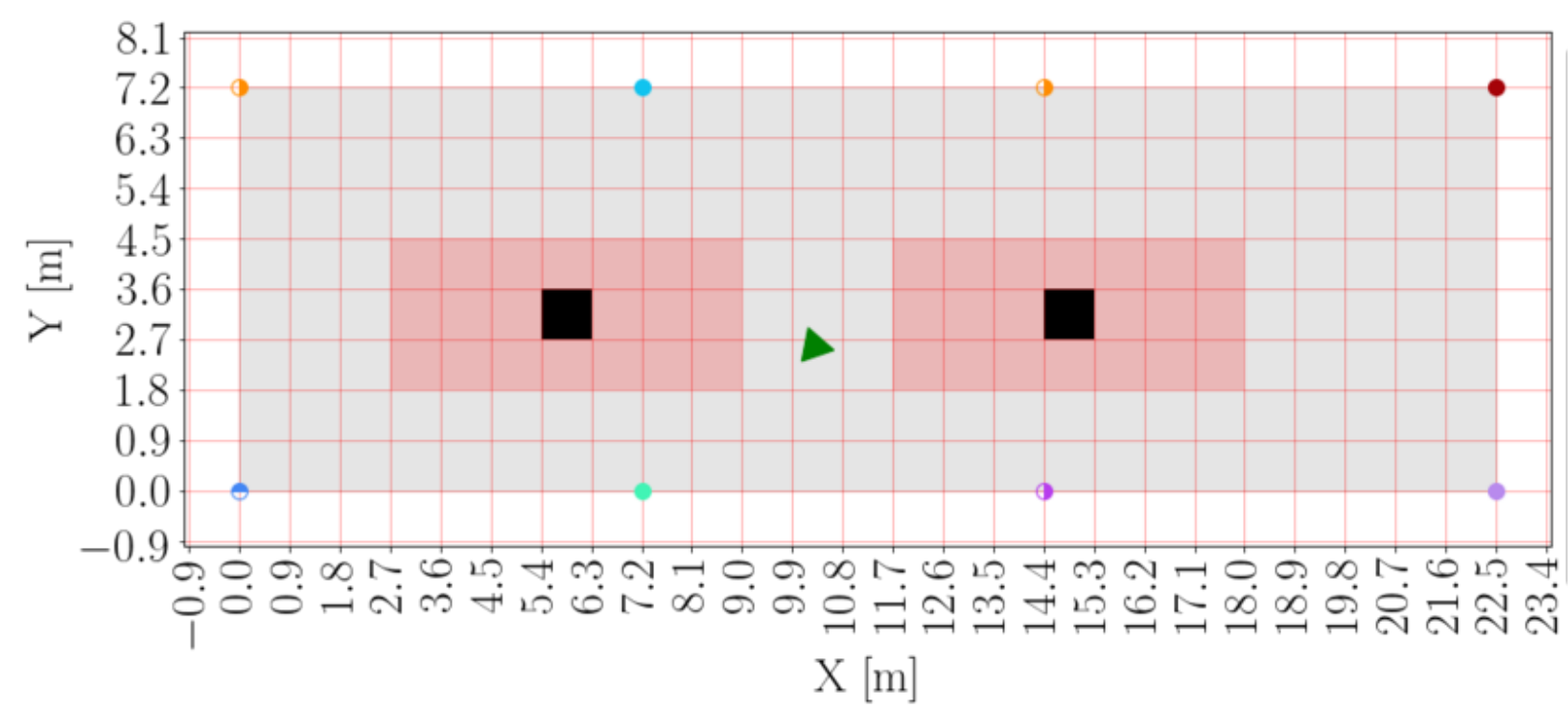

Figure 5.1: Grey grid cells demonstrate the localization environment $\Omega$. Grey triangle is the pose of the agent. The circles represent the access points while black regions depict the support columns. The grid cells marked with red color represent the regions where the fingerprints corresponding to the grid cells in shadowing effect are contributed into parameter estimation.

\subsubsection{Structure of the Environment}

The experimentation was conducted in Hancock Hall, Virginia Tech, where the environment can be considered empty. Thus, it is expected to LOS propagation be dominant in the environment. The environment contains two support columns inside and it is closed from three edges with cinder block walls while the last edge was open to another open area. The environment is 22.5 by 7.2 meters, in which 8 access points running at $900 \mathrm{MHz}$ were placed. Figure 5.3 provides a panoramic photograph of the experimentation site with obstructions were clearly depicted. 


\section{Grid-cell Size Determination}

The characteristics of the radio modules used plays a significant role in the the determination of the grid-cell size. Fraunhofer distance was considered to determine the size of the gridcells such that the fingerprints were acquired within far-field propagation zone $(d>2 \lambda)$. In the near-field zone, which is described as the first two wavelength equivalent distance from the transmitting antenna, the radio waves show fast-fading characteristics, while both SCPL and LDPL models approximate the slow-fading characteristics of the radio. Therefore, the grid-cell size was chosen such that the fingerprints will not be collected in near-field zone.

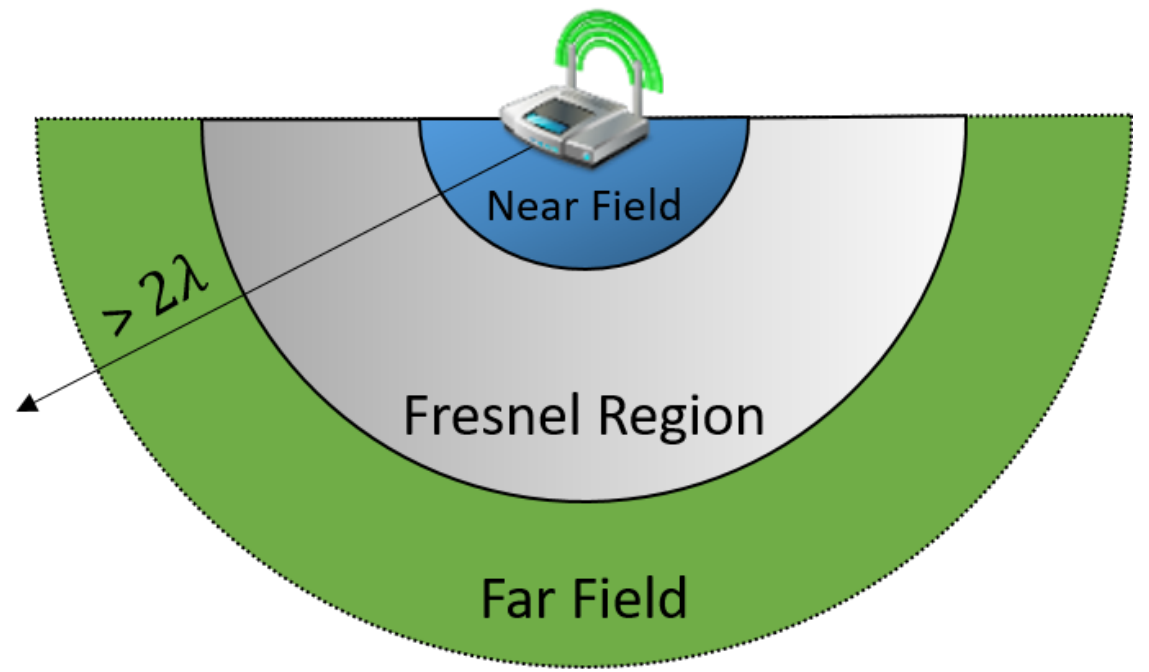

Figure 5.2: Radio waves show different characteristics after being emitted by the transmitting antenna as a function of distance. In Fraunhofer region $(d>2 \lambda)$, the radio waves display more steady characteristics than Fresnel region.

The grid-cell size determined as 0.9 by 0.9 meters as the radios, used in the experimentations, were running at $900 \mathrm{MHz}(\lambda=0.33$ meters $)$. Figure 5.1 represents the grid-cell representation of the environment, and the location of the access points. 


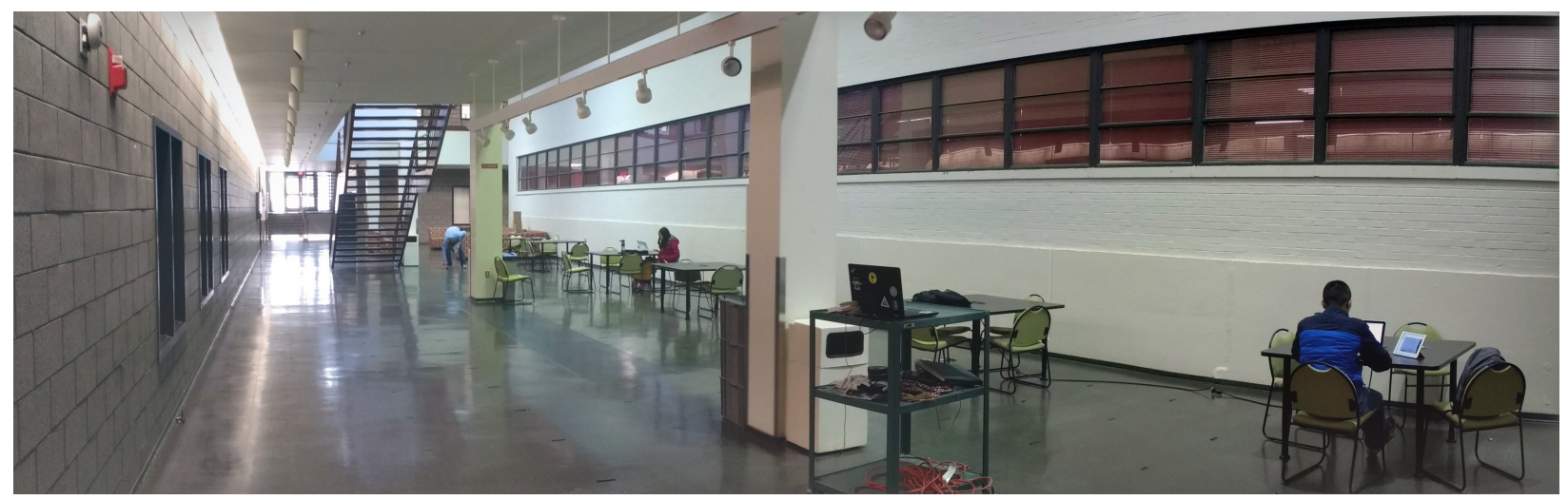

Figure 5.3: Panaromic photograph of the experimentation site

\subsubsection{Radio Modules}

\subsubsection{Dataset}

For each grid-cell, at least 6 repeated fingerprints were collected from all access points, and each grid-cell was visited at least 3 times. Therefore, 1543 fingerprint vectors from 8 access points were obtained along with the grid locations. For each grid-cell, there exist a mean of 7.715 measurements while the standard deviation of the measurements of grid-cells is 3.77 , from which one might conclude that the measurement space is not biased over locations.

\subsection{Experiments}

\subsubsection{Path Loss Estimation with SCPL}

The localization performance of the proposed technique heavily depends on path loss estimation ability. Thus, a significant attention has been put into this estimation ability. However, the trade off between generalization and accuracy needs to be resolved. The radio waves propagate with different characteristics depending on the wavelength of the wave and the 
obstruction size. In order to quantitatively assess the path loss estimation, two different radios working at wavelength different frequency, $900 \mathrm{MHz}$ and $2.4 \mathrm{GHz}$, is employed.

\section{$900 \mathrm{MHz}$ Channel}

In order to assess the propagation characterization ability of LDPL and SCPL, both of the techniques were first employed $900 \mathrm{MHz}$ frequency band.
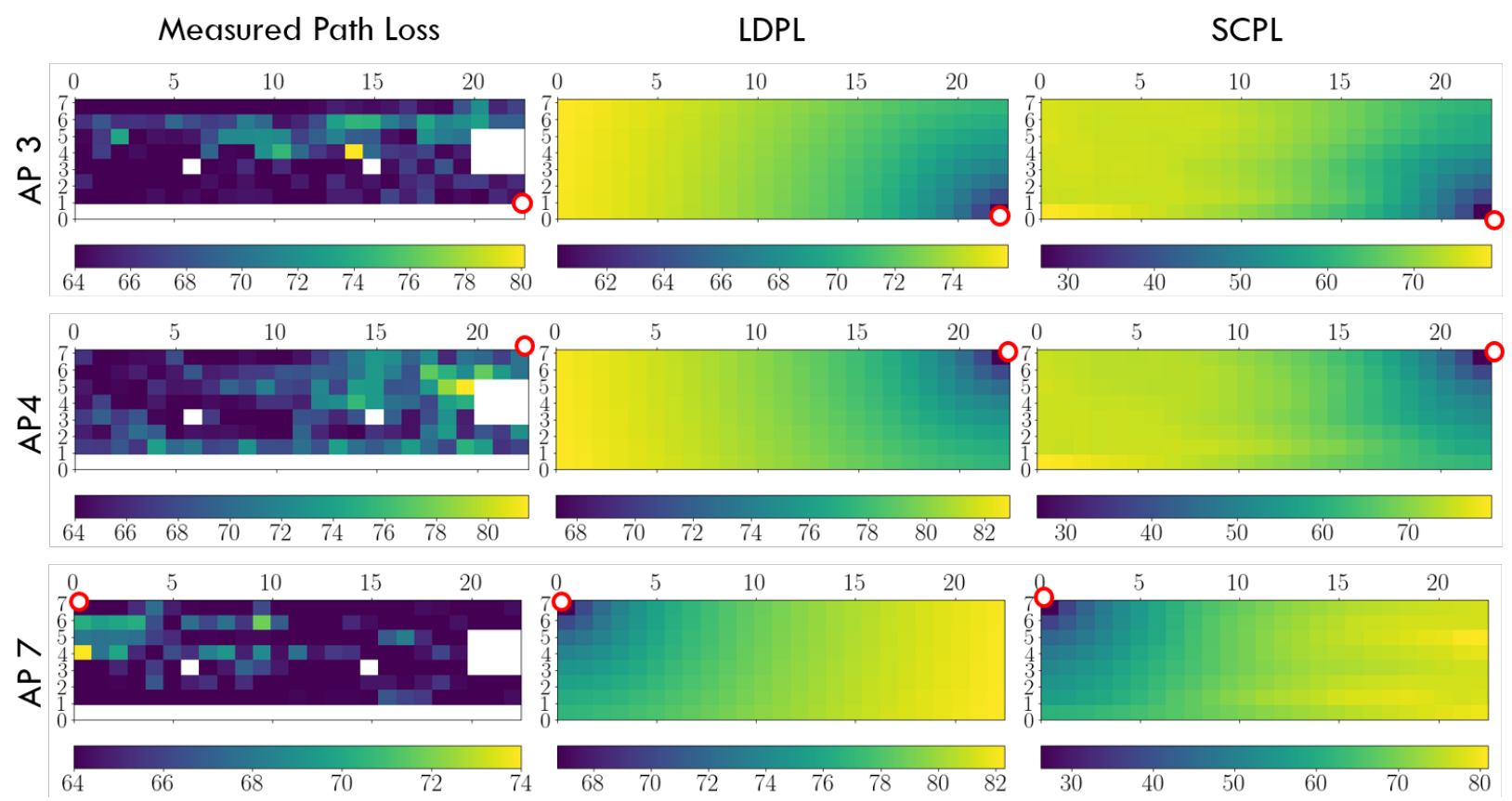

Figure 5.4: Measured mean path loss of each grid cell with different access points placed in the environment. The access point locations marked with the circles in the figure.

Figure 5.4 depicts the actual measurements and the estimations provided by LDPL and SCPL techniques. The first column represents the mean path loss measurements of each grid cell for access points 3,4 and 7 whose locations were marked with circles on the figure. As can be seen in the figure, access point 7 results in higher path loss measurements than access points 3 and 4 . In the light of this observation, one might conclude that the path loss measurements not only vary based on location, but also does the access point location 
contributes to this variation.

Since LDPL characterizes the propagation in a global manner, the estimations provided by this technique yields smooth power decay as the distance from the access points increases. This approaches fails to capture the propagation irregularities posed by the environment. On the other hand, due to local attenuation profiling nature of SCPL, it provided a larger range of estimations. This observation can be made with the color bar of each figure.

Figure 5.5 depicts the path loss estimation error of LDPL and SCPL at $900 \mathrm{MHz}$ frequency channel. As can be seen in the figure the path loss estimation error of LDPL technique is a function of distance. As the distance between the access point and the grid cell increases, LDPL seems to display poorer propagation characterization ability.
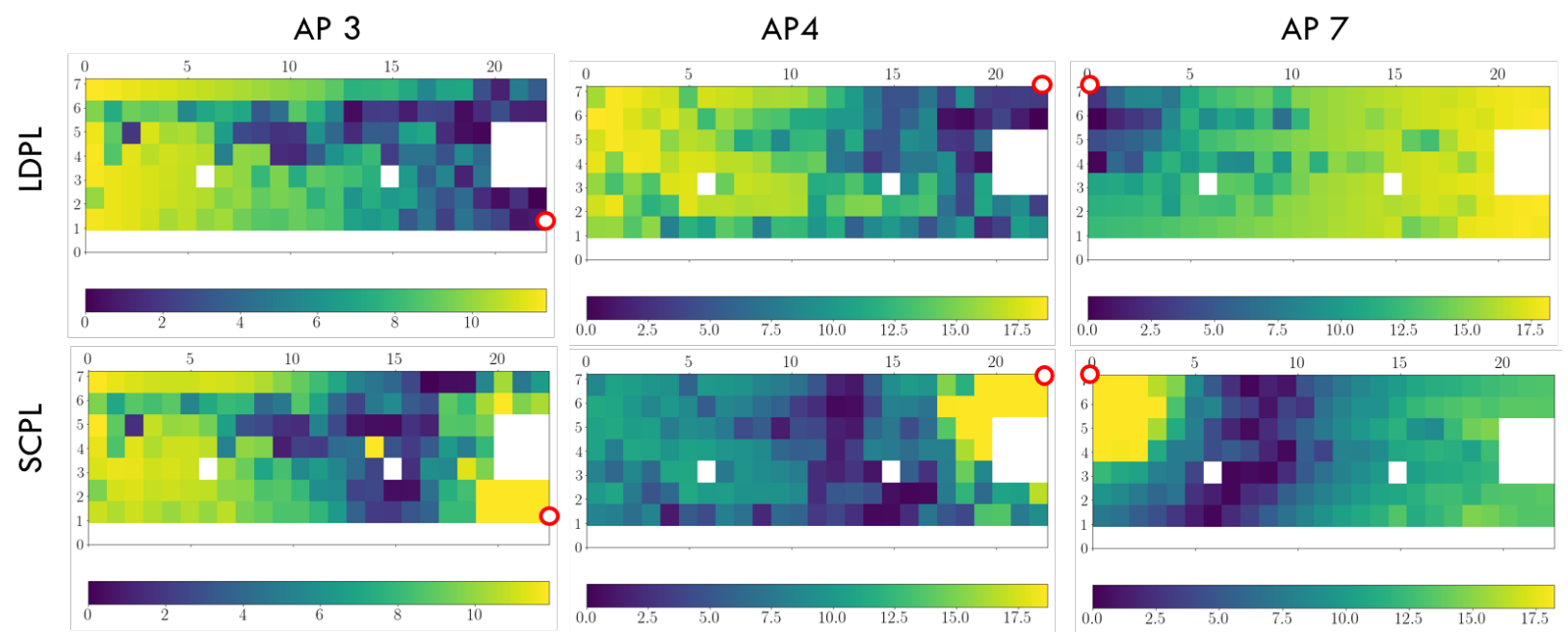

Figure 5.5: Path Loss Estimation Error of Access Points 3,4,7

While the path loss estimation of LDPL as a function of distance, SCPL yields to more complicated path loss estimations errors due to its local attenuation profiling nature. However, from the figure it can be seen that, SCPL tends to have larger error margins in the near field of the access point. 


\section{$2.4 \mathrm{GHz}$ Channel}

In order to assess the propagation characterization ability of LDPL and SCPL, both of the techniques were also employed at $2400 \mathrm{MHz}$ frequency band.

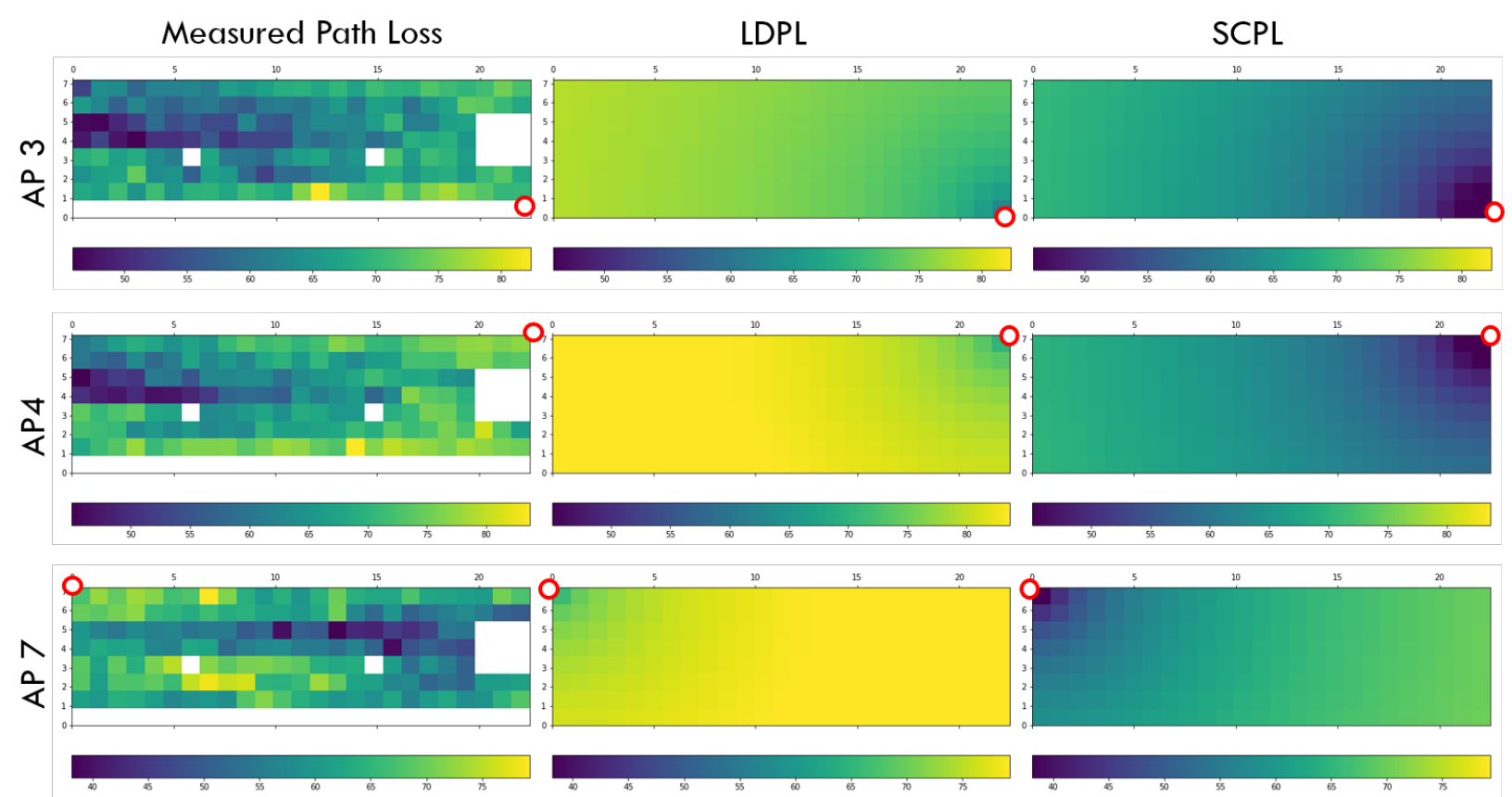

Figure 5.6: Measured mean path loss of each grid cell with different access points placed in the environment. The access point locations marked with the circles in the figure.

As can be seen in the Figure 5.6, path loss measured at $2400 \mathrm{MHz}$ frequency channel is significantly is bigger than $900 \mathrm{MHz}$. This increase in the path loss is due to the phenomenon that the radio waves get attenuated if the frequency of the signal increases (i.e. shorter wavelength). The first column of the figure represents the mean path loss measurements of each grid cell for the same access point employed in the analysis of $900 \mathrm{MHz}$ whose locations marked with circles on the figure. Similar to analysis of path loss estimation at $900 \mathrm{MHz}$, different access points results in significantly different path loss measurements depending on their locations.

Figure 5.7 depicts the path loss estimation error of LDPL and SCPL at $2400 \mathrm{MHz}$ 
frequency channel. Similar to path loss estimation analysis conducted with $900 \mathrm{MHz}$ radio modules, path loss estimation error of LDPL technique yields larger error as the the distance between the access point and the grid cell increases.
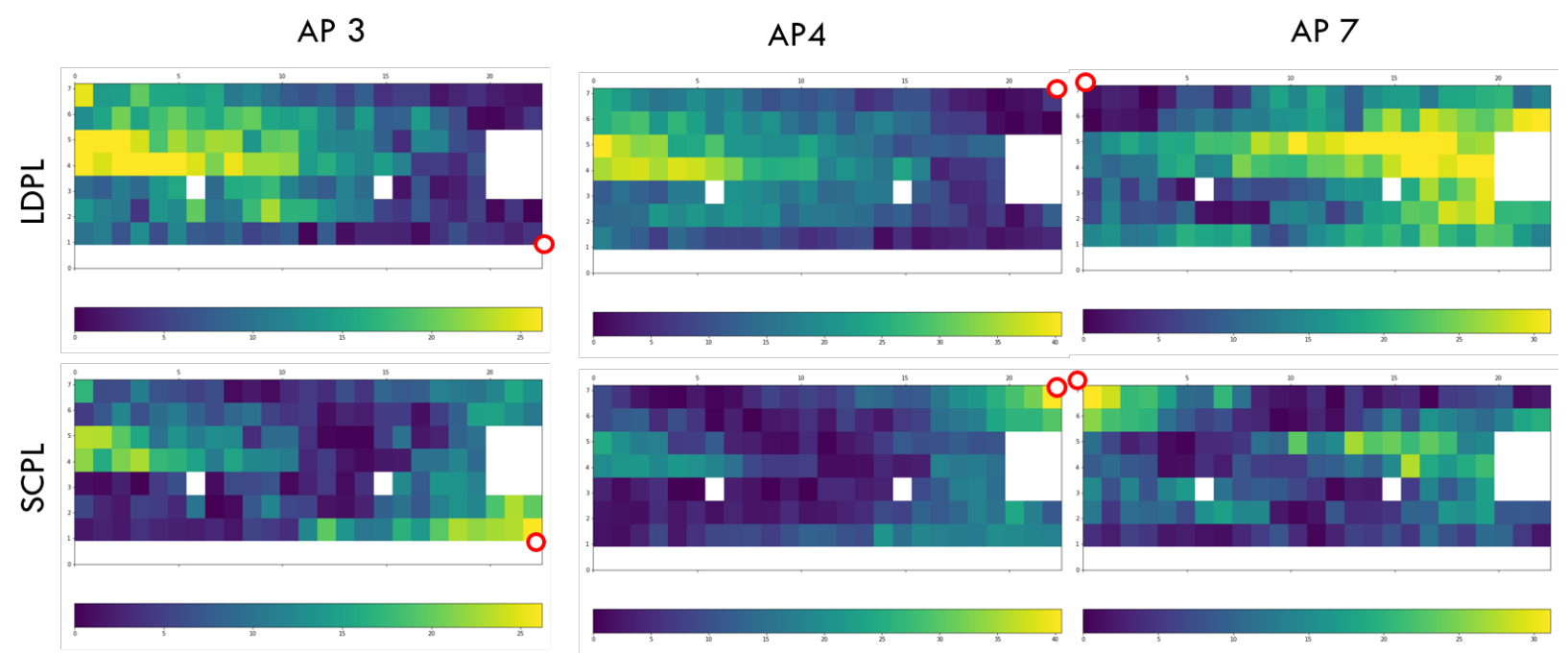

Figure 5.7: Path Loss Estimation Error of Access Points 3,4,7 at $2400 \mathrm{MHz}$ frequency channel

While the path loss estimation of LDPL as a function of distance, SCPL yields to more complicated path loss estimations errors due to its local attenuation profiling nature. However, from the figure it can be seen that, SCPL tends to have larger error margins in the near field of the access point.

\subsubsection{Analysis of SCPL Model Parameters}

\section{Effect of N-LOS on Model Parameters}

In Figure 5.1, grid-cells marked with red color represent the region where support columns affected during the parameter estimation, while the colums are marked with black. It is expected to observe an increase in model parameters' values due to likeliness of N-LOS propagation around the columns. In the light of this analysis, it is expected to observe an 
increase in the estimated the path loss exponent where N-LOS propagation path occurs; Figure 5.8 validates the expectation.

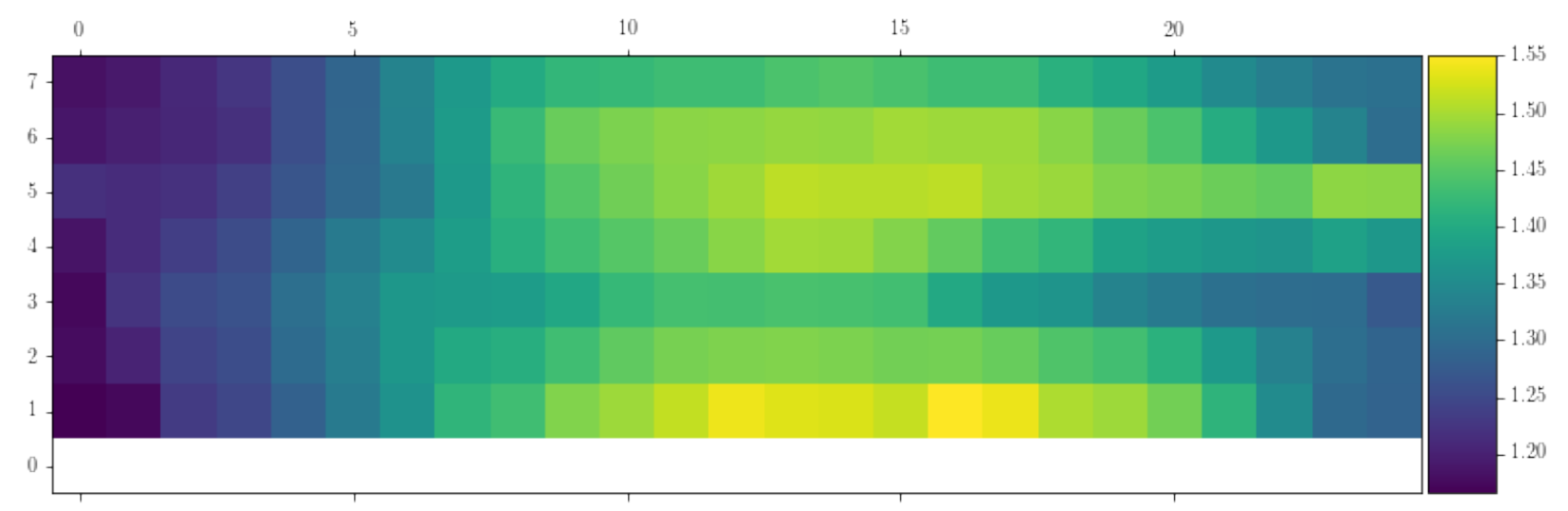

Figure 5.8: Estimated path loss parameters for each grid cells. As it can be seen, the grid cells neighboring obstructions tend to have larger path loss exponent, which shows attenuation in these vicinities have more impact on the localization.

\section{Analysis of Parameter Selection}

As described in Chapter 4, a three layer feed-forward neural network was employed with which the parameters of model $\left(n_{g}, \alpha_{g}, \sigma_{g}\right)$ are chosen. The network is trained with Stochastic Gradient Descent algorithm with Nesterov momentum updates. The implementation is implemented with Keras [50] library which uses Tensorflow [51] as the backend. Overall the neural network was able to choose the model parameters with an error of 0.0082 (MSE).

\subsubsection{Analysis of Estimated Separation Distance}

In order to analyze the efficacy of the SCPL model, first the separation distance estimation is analyzed. Given a vector fingerprints collected from all observable access points at an unknown position, SCPL and LDPL are separately used to estimate the separation distance from all access points. The error metric for each fingerprint vector is given below. 


$$
e_{1 d}=\frac{1}{n_{s} n_{a n}} \sum_{i=1}^{n_{s}} \sum_{j=1}^{n_{a n}}\left\|\hat{d}_{i}^{j}-d_{i}^{j}\right\|
$$

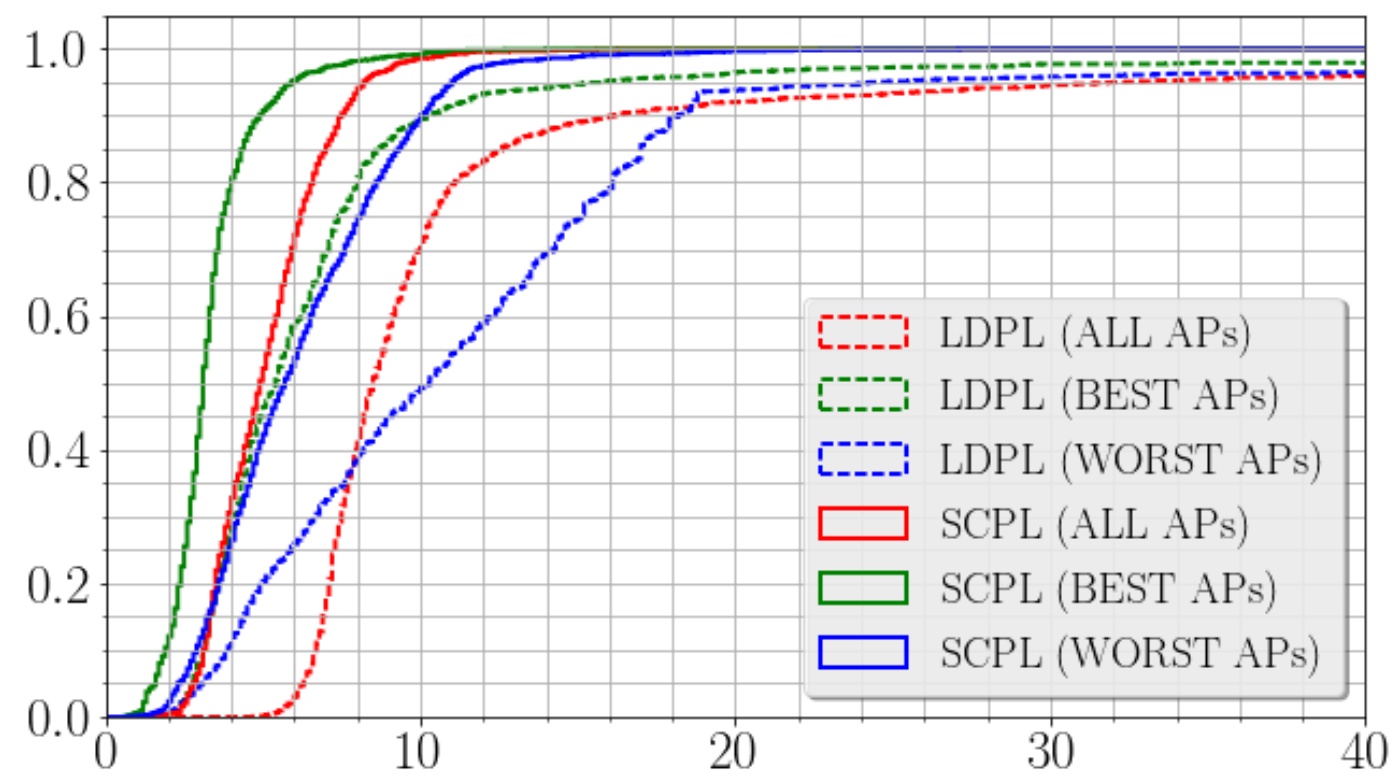

Figure 5.9: Cumulative distribution function of range estimation errors

Table 5.1 tabulates the estimated separation distance error for LDPL, SCPL models. The first column represents the overall error statistics of the LDPL model, while second column that of SCPL with perfect parameter selection. The third column of the Table 5.1 represents the case where model parameters were chosen with the neural network. As shown in Figure 5.9 and Table 5.1, SCPL is able to localize the target with 5.2 meters mean localization performance, while LDPL achieves 14.98 meters.

As well as the mean errors, the errors distribution of both algorithm approximated with a normal distribution by employing Central Limit Theorem (CLT). The last row of the Table 5.1 denotes the $95 \%$ level confidence intervals for each algorithm. While LDPL achieves range estimation between 7.91 and 22.05 meters, the confidence interval attained 
Table 5.1: Comparison of Errors in Separation Distance Estimation

\begin{tabular}{llll}
\hline Error Metric & LDPL & SCPL (w/o NN) & SCPL (w/ NN) \\
\hline Mean Error $[\mathrm{m}]$ & 14.98 & $\mathbf{5 . 1 9}$ & 5.20 \\
\hline Median Error $[\mathrm{m}]$ & 7.17 & $\mathbf{4 . 1}$ & 4.11 \\
\hline Std. Dev. $[\mathrm{m}]$ & 141.79 & 4.68 & $\mathbf{4 . 6 1}$ \\
\hline Confidence Interval & {$[7.91,22.05]$} & {$[\mathbf{4 . 9 7}, \mathbf{5 . 4 3}]$} & {$[4.98,5.43]$} \\
\hline
\end{tabular}

with SCPL is between 4.98 , and 5.43 meters.

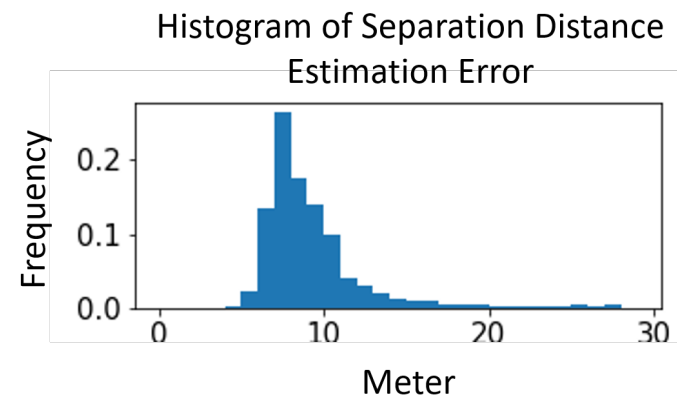

(a) Histogram of Separation Distance Error for LDPL

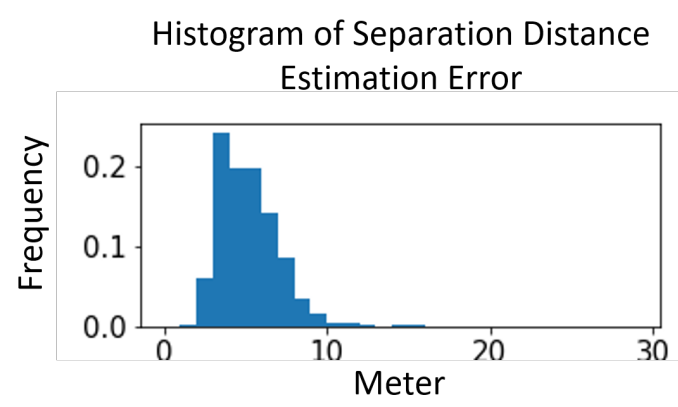

(b) Histogram of Separation Distance Error for SCPL

Figure 5.10: Left: Histogram of Separation Distance Error for LDPL

Right: Histogram of Separation Distance Error for SCPL

\subsubsection{Analysis of Target Localization}

The performance of SCPL model was also analyzed after trilateration technique is employed.

The error metric used in the analysis is given in Equation (5.2).

$$
e_{2 d}=\frac{1}{n_{s} n_{a n}} \sum_{i=1}^{n_{s}} \sum_{j=1}^{n_{a n}}\left\|\hat{x}_{i}^{j}-x_{i}^{j}\right\|_{2}
$$

As shown in Table 5.2, after triangulation, the performance of both LDPL and SCPL 


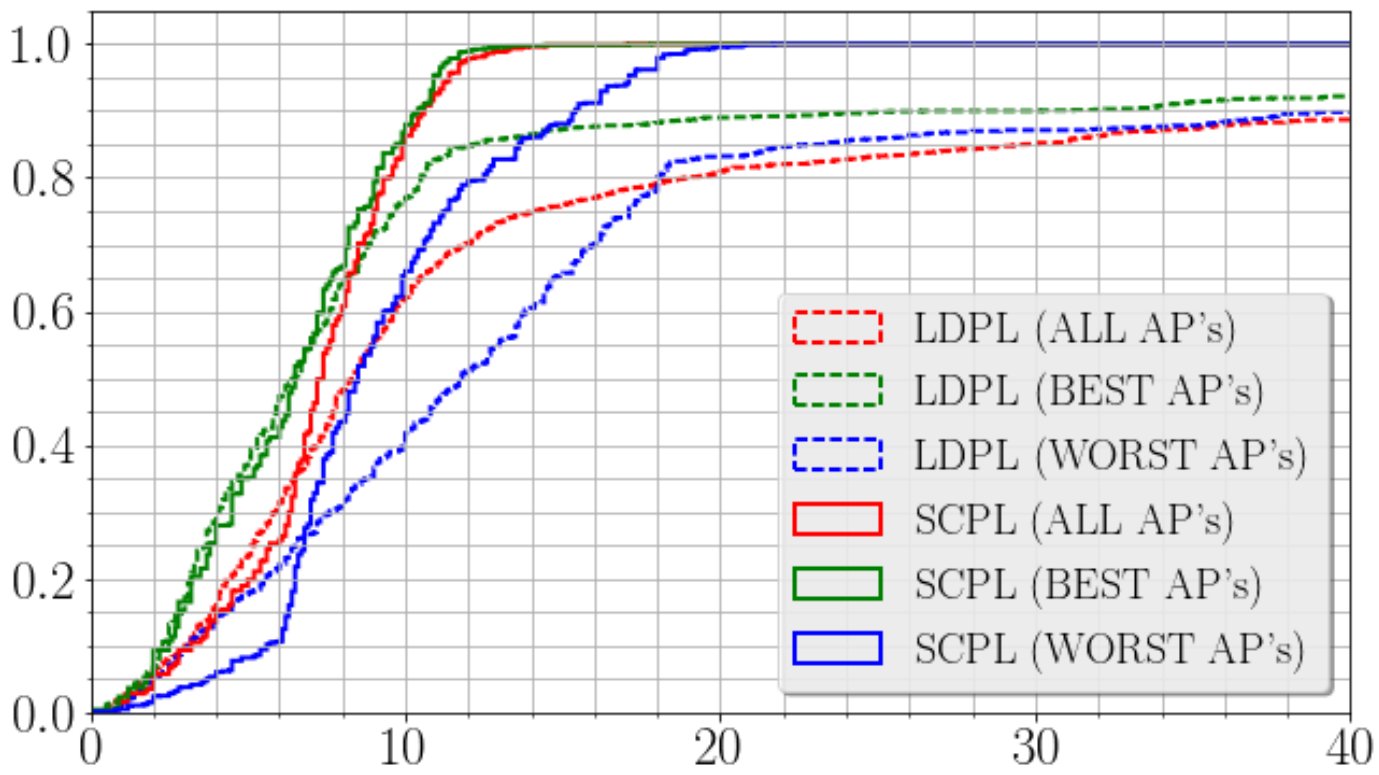

Figure 5.11: Cumulative distribution function of target localization error.

degraded. By using a neural network to choose model parameters, SCPL achieved mean 7.31 localization in target location estimation. On the other hand, LDPL was able to localize the target with 8.66 mean localization error. Figure 5.11 demonstrates the cumulative distribution function of the target localization error across the dataset. As can be seen in Figure $5.11,80 \%$ of the errors below 13 meters.

Table 5.2: Comparison of Errors in Target Localization

\begin{tabular}{l|c|cc}
\hline & LDPL & \multicolumn{2}{c}{ SCPL } \\
\hline Error Metric & Trilateration & Trilateration & Trilateration + Grid Localization \\
\hline Mean Error $[\mathrm{m}]$ & 5932.56 & 10.05 & $\mathbf{7 . 2 7}$ \\
\hline Median Error $[\mathrm{m}]$ & 8.66 & 8.11 & $\mathbf{7 . 1}$ \\
\hline Std. Dev. [m] & 124988 & 12.60 & $\mathbf{2 . 7 2}$ \\
\hline Confidence Interval & {$[-303.82,12168]$} & {$[9.46,10.99]$} & {$[\mathbf{7 . 1 4}, \mathbf{7 . 4 1}]$} \\
\hline
\end{tabular}




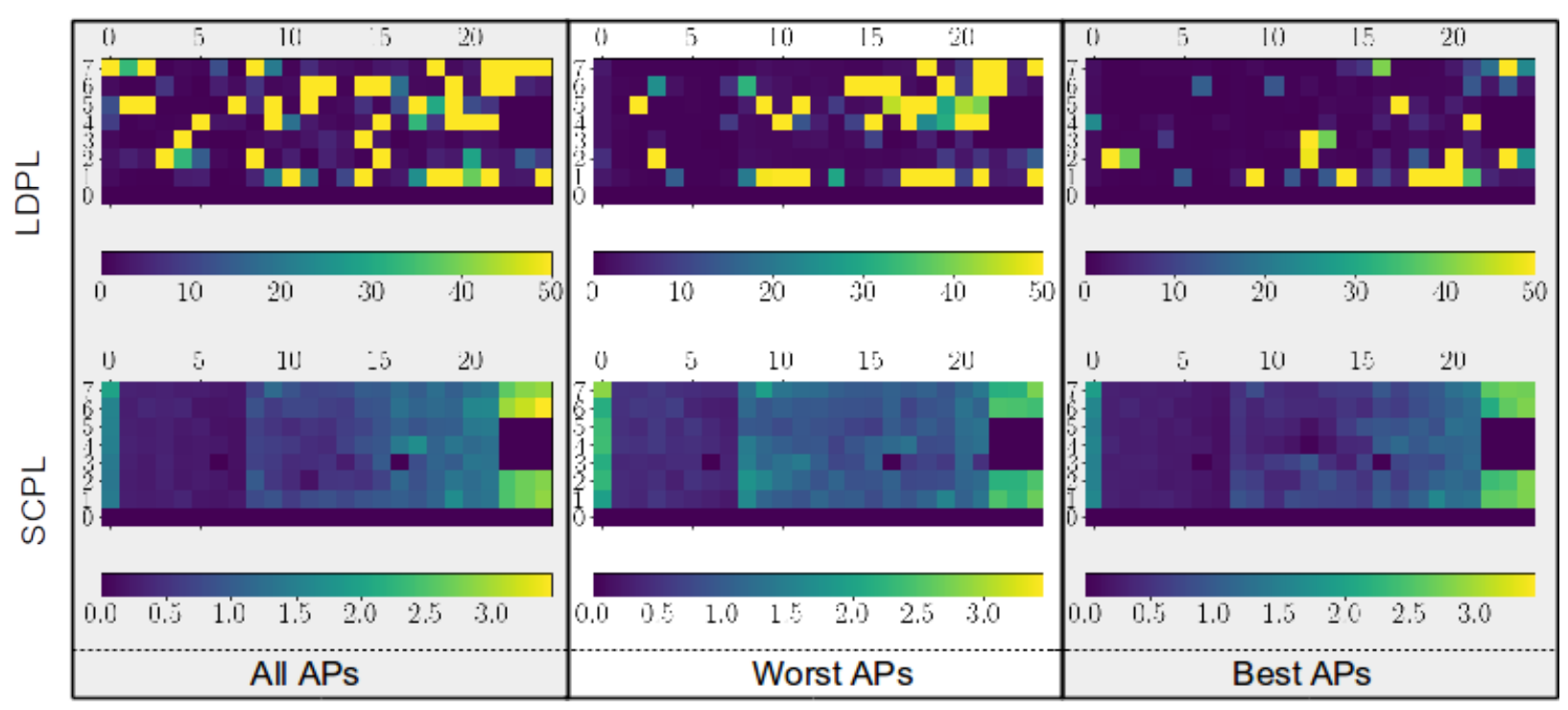

Figure 5.12: Error distribution of both algorithms over grid cells.
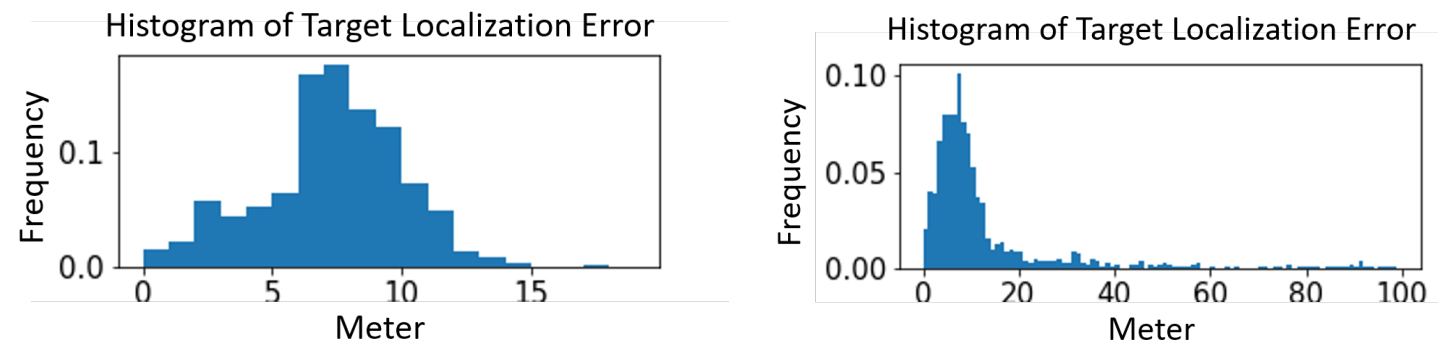

(a) Histogram of Target Localization Error for LDPL

(b) Histogram of Target Localization Error for SCPL

Figure 5.13: Left: Histogram of Target Localization Error for LDPL

Right: Histogram of Target Localization Error for SCPL

\subsubsection{Access Point Analysis}

In order to assess effect of the access points location in the separation distance estimation, the error contribution of each access point in the target localization is also analyzed. Table 5.3 tabulates the mean error contribution of each access point across the dataset. The table clearly shows that the distribution of error contribution of each access point is not uniform. 
Table 5.3: Error Statistics of Access Points in Separation Distance Estimation

\begin{tabular}{lccc}
\hline Access Point & LDPL & SCPL (w/o NN) & SCPL (w/ NN) \\
\hline Access Point 0 & 33.33 & 7.05 & 6.63 \\
\hline Access Point 1 & 7.86 & 3.55 & 3.52 \\
\hline Access Point 2 & 6.77 & 2.66 & 3.13 \\
\hline Access Point 3 & 19.14 & 6.25 & 6.26 \\
\hline Access Point 4 & 13.76 & 6.91 & 6.99 \\
\hline Access Point 5 & 18.1 & 4.96 & 5.34 \\
\hline Access Point 6 & 9.6 & 3.62 & 3.57 \\
\hline Access Point 7 & 11.28 & 6.6 & 6.15 \\
\hline
\end{tabular}

In order to further quantify the effect, the fingerprints of the best 3 access points employed to determine to separation distance. The selection of the best 3 and worst access points was rather straight forward. All 3 combinations of all (8) access points measurements are employed to estimate the separation distance while the remaining fingerprints were discarded. With the combinatorial analysis, access points denoted with 1, 2, 6 (see Figure 5.1) found to be the least contributor in the error, while the combination of $3,4,5$ seem to perfom the worst in our dataset.

By only using the access points found to be more reliable, the error deviation of LDPL model reduced significantly, while the mean error of LDPL and SCPL models decreased by $\% 46$ and \%34, respectively. On the other hand, worst-3 analysis demonstrates that the mean error of LDPL and SCPL models increased $13 \%$ and 20\%, respectively. 


\begin{tabular}{|c|c|c|c|}
\hline \multicolumn{4}{|c|}{ Best-3 Access Points } \\
\hline Error Metric & LDPL & $\mathrm{SCPL}(\mathrm{w} / \mathrm{o} \mathrm{NN})$ & $\mathrm{SCPL}(\mathrm{w} / \mathrm{NN})$ \\
\hline Mean Error $[\mathrm{m}]$ & 8.07 & 3.28 & 3.39 \\
\hline Median Error $[\mathrm{m}]$ & 5.03 & 2.84 & 2.87 \\
\hline Std. Dev. [m] & 27.84 & 2.79 & 2.82 \\
\hline Confidence Interval & {$[6.69,9.47]$} & {$[3.14,3.42]$} & {$[3.25,3.53]$} \\
\hline \multicolumn{4}{|c|}{ Worst-3 Access Points } \\
\hline Error Metric & LDPL & $\mathrm{SCPL}(\mathrm{w} / \mathrm{o} \mathrm{NN})$ & $\mathrm{SCPL}(\mathrm{w} / \mathrm{NN})$ \\
\hline Mean Error [m] & 16.99 & 6.03 & 6.25 \\
\hline Median Error $[\mathrm{m}]$ & 9.19 & 5.01 & 5.1 \\
\hline Std. Dev. $[\mathrm{m}]$ & 108.189 & 5.19 & 5.21 \\
\hline Confidence Interval & {$[11.60,22.39]$} & {$[5.77,6.30]$} & {$[5.99,6.51]$} \\
\hline
\end{tabular}

Table 5.4: Comparison of Error Statistics in Separation Distance Estimation with Best 3 and Worst 3 access points

\subsection{Summary}

This section has provided the experiments conducted for analyzing the overall performance of the proposed path loss model, i.e. SCPL and the grid based radiolocation based on it.

The experimentation was conducted in Hancock Hall, Virginia Tech. In order to elaborate on the performance of the proposed technique, the experiments were conducted at two different frequency channels, $900 \mathrm{MHz}$ and $2.4 \mathrm{GHz}$. First, path loss estimation, i.e. radio wave propagation characterization ability, of SCPL was analyzed. In the light of the results of this experiment, SCPL seems to more robust to multi-path effect then LDPL. In the light of the comprehensive experiments conducted, SCPL displays better propagation characterization thanks to the local attenuation profiling technique employed than LDPL which employs global attenuation profiling for indoor environments. It was also observed that LDPL tends to show less accurate characterization if the distance of the grid cell is 
further away from the access point, while SCPL displays less accurate characterization in near field. From this observation, SCPL can be employed in larger environments with fewer access points.

The second analysis was conducted for the purpose of observing the model parameters in the presence of the obstructions. While LDPL was not able to characterize the local attenuation sources, SCPL seemed to adapt the model such that local attenuation sources of the environment would not affect the global performance.

As for the analysis localization performance, LDPL and SCPL was employed in two different localization technique. The model parameters of SCPL for each grid cell was learned with a feed-forward neural network. The trained neural network was then used to infer the model parameters in the localization phase. This scheme of parameter acquisition for SCPL seem to affect the overall localization in minuscule levels. By using the trained neural network, SCPL achieved mean 7.31 localization in target location estimation. On the other hand, LDPL was able to localize the target with 8.66 mean localization error.

Lastly, the effects of the access point placement was analyzed on the overall proposed localization method. In the light of the experiments, LDPL yielded widely varying results depending on the access point selected to localize the agent. This variation in the localization error can be attributed to the fact that LDPL yields less accurate path loss estimations as the distance between the agent and the access point increases. 


\section{Chapter 6}

\section{Conclusions and Future Work}

This thesis has presented a grid-based indoor radiolocation technique based on the SCPL. The proposed technique describes localization environments with a grid cell structure, and the wave propagation is estimated for each grid cell so that the obstructions can only effect the propagation model in a local vicinity. The grid cell representation of the environment is furthered incorporated it into localization by representing the results as probability distribution over the grid cells.

This ability enabled SCPL to capture the attenuation caused by the environment, and to significantly improve (up to $44 \%$ ) the localization performance in range estimations in comparison to LDPL. In the light of the experimentations, SCPL display more robustness to error caused by the N-LOS fingerprints than LDPL. Overall, the proposed grid-based indoor radiolocation technique yielded up to $18 \%$ (median) target localization improvement over LDPL based radiolocation technique. Results have demonstrated the applicability of the proposed approach in unstructured environments.

This paper has focused on a grid-based indoor radiolocation technique based on SCPL 
which can account for obstruction dissimilarity of the environment. However, much work is still left open.

As the analysis shows, not every access point results in the same extent of error; further investigation must be done to quantify the error contribution of each access point from which an active access point selection can be employed to further reduce the localization error.

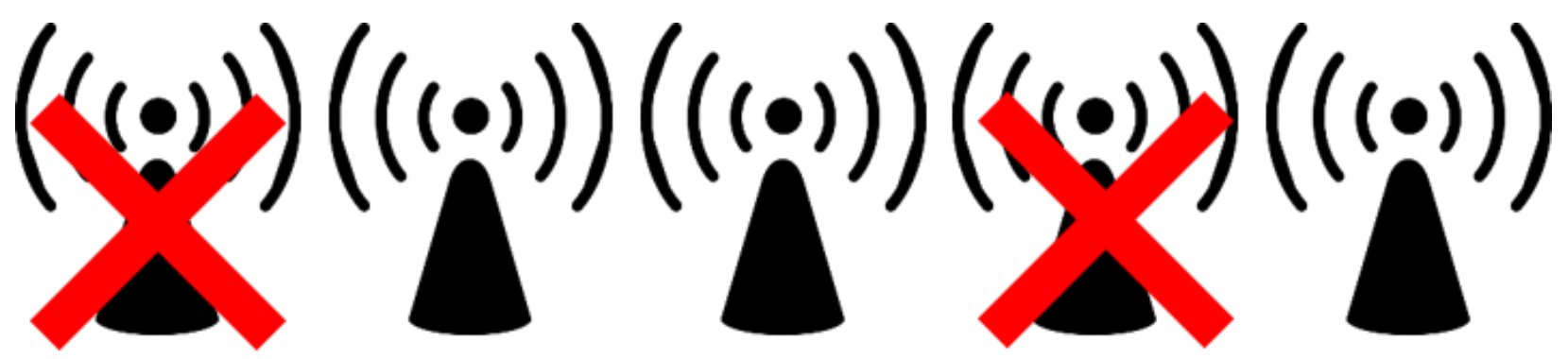

Figure 6.1: An active access point selection algorithm based on location information would inherently improve localization performance of the proposed algorithm.

Along with the access point selection algorithm, proposed localization technique can be incorporated into a state estimation framework. By doing so, agents can be tracked over an indoor environment and model inaccuracies can be accounted for. Due to grid-based formulation of the proposed technique, Grid-based recursive Bayesian framework seems to be a natural fit for such implementations.

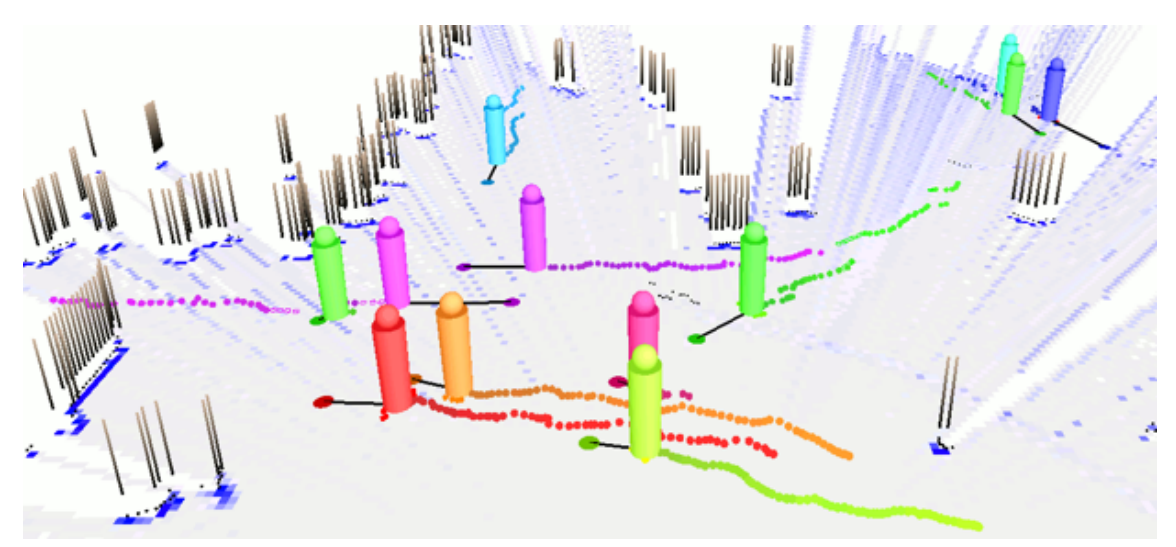

Figure 6.2: Tracking algorithms based on probabilistic state estimation methods would improve localization accuracy as well as provide a potential to tackle model inaccuracies. 


\section{Bibliography}

[1] S. Goswami, Indoor location technologies. Springer Science \& Business Media, 2012.

[2] G. N. DeSouza and A. C. Kak, "Vision for mobile robot navigation: A survey," IEEE transactions on pattern analysis and machine intelligence, vol. 24, no. 2, pp. 237-267, 2002.

[3] K. Sato, T. Manabe, J. Polivka, T. Ihara, Y. Kasashima, and K. Yamaki, "Measurement of the complex refractive index of concrete at $57.5 \mathrm{ghz}$," IEEE Transactions on Antennas and Propagation, vol. 44, no. 1, pp. 35-40, 1996.

[4] S. He and S.-H. G. Chan, "Wi-fi fingerprint-based indoor positioning: Recent advances and comparisons," IEEE Communications Surveys \& Tutorials, vol. 18, no. 1, pp. 466490, 2016.

[5] V. Honkavirta, T. Perala, S. Ali-Loytty, and R. Piché, "A comparative survey of wlan location fingerprinting methods," in Positioning, Navigation and Communication, 2009. WPNC 2009. 6th Workshop on. IEEE, 2009, pp. 243-251.

[6] A. M. Hossain and W.-S. Soh, "A survey of calibration-free indoor positioning systems," Computer Communications, vol. 66, pp. 1-13, 2015. 
[7] H. Liu, H. Darabi, P. Banerjee, and J. Liu, "Survey of wireless indoor positioning techniques and systems," IEEE Transactions on Systems, Man, and Cybernetics, Part C (Applications and Reviews), vol. 37, no. 6, pp. 1067-1080, 2007.

[8] J. S. Seybold, Introduction to RF propagation. John Wiley \& Sons, 2005.

[9] A. Goldsmith, Wireless communications. Cambridge university press, 2005.

[10] J. B. Andersen, T. S. Rappaport, and S. Yoshida, "Propagation measurements and models for wireless communications channels," IEEE Communications Magazine, vol. 33, no. 1 , pp. 42-49, 1995.

[11] P. Series, "Propagation data and prediction methods for the planning of indoor radiocommunication systems and radio local area networks in the frequency range $900 \mathrm{mhz}$ to 100 ghz," ICU: Genève, Switzerland, 2012.

[12] T. S. Rappaport et al., Wireless communications: principles and practice. prentice hall PTR New Jersey, 1996, vol. 2.

[13] M. Vossiek, L. Wiebking, P. Gulden, J. Wieghardt, C. Hoffmann, and P. Heide, "Wireless local positioning," IEEE microwave magazine, vol. 4, no. 4, pp. 77-86, 2003.

[14] B. Denis, J. Keignart, and N. Daniele, "Impact of nlos propagation upon ranging precision in uwb systems," in Ultra Wideband Systems and Technologies, 2003 IEEE Conference on. IEEE, 2003, pp. 379-383.

[15] L. C. Mak, "Non-line-of-sight localisation of a sound source," School of Mechanical and Manufacturing Engineering, 2009.

[16] S. Venkatraman and J. Caffery, "Statistical approach to non-line-of-sight bs identification," in Wireless Personal Multimedia Communications, 2002. The 5th International Symposium on, vol. 1. IEEE, 2002, pp. 296-300. 
[17] P.-C. Chen, "A non-line-of-sight error mitigation algorithm in location estimation," in Wireless Communications and Networking Conference, 1999. WCNC. 1999 IEEE, vol. 1. IEEE, 1999, pp. 316-320.

[18] L. Cong and W. Zhuang, "Nonline-of-sight error mitigation in mobile location," IEEE Transactions on Wireless Communications, vol. 4, no. 2, pp. 560-573, 2005.

[19] J. Borras, P. Hatrack, and N. B. Mandayam, "Decision theoretic framework for nlos identification," in Vehicular Technology Conference, 1998. VTC 98. 48th IEEE, vol. 2. IEEE, 1998, pp. 1583-1587.

[20] D. Vasisht, S. Kumar, and D. Katabi, "Decimeter-level localization with a single wifi access point." in NSDI, 2016, pp. 165-178.

[21] M. Kossel, H. Benedickter, R. Peter, and W. Bachtold, "Microwave backscatter modulation systems," in Microwave Symposium Digest. 2000 IEEE MTT-S International, vol. 3. IEEE, 2000, pp. 1427-1430.

[22] M. M. Atia, A. Noureldin, and M. J. Korenberg, "Dynamic online-calibrated radio maps for indoor positioning in wireless local area networks," IEEE Transactions on Mobile Computing, vol. 12, no. 9, pp. 1774-1787, 2013.

[23] V. Bahl and V. Padmanabhan, "Radar: An in-building rf-based user location and tracking system." Institute of Electrical and Electronics Engineers, Inc., March 2000. [Online]. Available: https://www.microsoft.com/en-us/research/publication/ radar-an-in-building-rf-based-user-location-and-tracking-system/

[24] Z.-l. Wu, C.-h. Li, J. K.-Y. Ng, and K. R. ph Leung, "Location estimation via support vector regression," IEEE Transactions on mobile computing, vol. 6, no. 3, pp. 311-321, 2007. 
[25] M. Youssef and A. Agrawala, "The horus wlan location determination system," in Proceedings of the 3rd international conference on Mobile systems, applications, and services. ACM, 2005, pp. 205-218.

[26] D. Fahed and R. Liu, "Wi-fi-based localization in dynamic indoor environment using a dynamic neural network," International Journal of Machine Learning and Computing, vol. 3, no. 1, p. 127, 2013.

[27] K. Trawiński, J. M. Alonso, and N. Hernández, "A multiclassifier approach for topologybased wifi indoor localization," Soft Computing, vol. 17, no. 10, pp. 1817-1831, 2013.

[28] P. Bahl, V. N. Padmanabhan, and A. Balachandran, "Enhancements to the radar user location and tracking system," Microsoft Research, vol. 2, no. MSR-TR-2000-12, pp. $775-784,2000$.

[29] H. Lim, L.-C. Kung, J. C. Hou, and H. Luo, "Zero-configuration, robust indoor localization: Theory and experimentation," Tech. Rep., 2005.

[30] K. Chintalapudi, A. Padmanabha Iyer, and V. N. Padmanabhan, "Indoor localization without the pain," in Proceedings of the sixteenth annual international conference on Mobile computing and networking. ACM, 2010, pp. 173-184.

[31] A. Goswami, L. E. Ortiz, and S. R. Das, "Wigem: A learning-based approach for indoor localization," in Proceedings of the Seventh COnference on emerging Networking EXperiments and Technologies. ACM, 2011, p. 3.

[32] Z. Chen, H. Zou, H. Jiang, Q. Zhu, Y. C. Soh, and L. Xie, "Fusion of wifi, smartphone sensors and landmarks using the kalman filter for indoor localization," Sensors, vol. 15, no. 1 , pp. $715-732,2015$. 
[33] F. Evennou and F. Marx, "Advanced integration of wifi and inertial navigation systems for indoor mobile positioning," Eurasip journal on applied signal processing, vol. 2006, pp. 164-164, 2006.

[34] J. Xiong and K. Jamieson, "Arraytrack: a fine-grained indoor location system," in Presented as part of the 10th USENIX Symposium on Networked Systems Design and Implementation (NSDI 13), 2013, pp. 71-84.

[35] M. Kotaru, K. Joshi, D. Bharadia, and S. Katti, "Spotfi: Decimeter level localization using wifi," in ACM SIGCOMM Computer Communication Review, vol. 45, no. 4. ACM, 2015, pp. 269-282.

[36] S. Kumar, E. Hamed, D. Katabi, and L. Erran Li, "Lte radio analytics made easy and accessible," in ACM SIGCOMM Computer Communication Review, vol. 44, no. 4. ACM, 2014, pp. 211-222.

[37] S. Kumar, S. Gil, D. Katabi, and D. Rus, "Accurate indoor localization with zero start-up cost," in Proceedings of the 20th annual international conference on Mobile computing and networking. ACM, 2014, pp. 483-494.

[38] J. Wang and D. Katabi, "Dude, where's my card?: Rfid positioning that works with multipath and non-line of sight," ACM SIGCOMM Computer Communication Review, vol. 43, no. 4, pp. 51-62, 2013.

[39] F. Adib and D. Katabi, See through walls with WiFi! ACM, 2013, vol. 43, no. 4.

[40] S. Sen, B. Radunovic, R. R. Choudhury, and T. Minka, "You are facing the mona lisa: spot localization using phy layer information," in Proceedings of the 10th international conference on Mobile systems, applications, and services. ACM, 2012, pp. 183-196. 
[41] J. Xiao, K. Wu, Y. Yi, and L. M. Ni, "Fifs: Fine-grained indoor fingerprinting system," in Computer Communications and Networks (ICCCN), 2012 21st International Conference on. IEEE, 2012, pp. 1-7.

[42] X. Wang, L. Gao, and S. Mao, "Phasefi: Phase fingerprinting for indoor localization with a deep learning approach," in Global Communications Conference (GLOBECOM), 2015 IEEE. IEEE, 2015, pp. 1-6.

[43] X. Wang, L. Gao, S. Mao, and S. Pandey, "Csi-based fingerprinting for indoor localization: A deep learning approach," IEEE Transactions on Vehicular Technology, vol. 66, no. 1, pp. 763-776, 2017.

[44] Y. Bengio, P. Lamblin, D. Popovici, and H. Larochelle, "Greedy layer-wise training of deep networks," in Advances in neural information processing systems, 2007, pp. 153-160.

[45] X. Cai, X. Li, R. Yuan, and Y. Hei, "Identification and mitigation of nlos based on channel state information for indoor wifi localization," in Wireless Communications $\&$ Signal Processing (WCSP), 2015 International Conference on. IEEE, 2015, pp. 1-5.

[46] H. T. Friis, "A note on a simple transmission formula," Proceedings of the IRE, vol. 34, no. 5, pp. 254-256, 1946.

[47] N. Balal, G. A. Pinhasi, and Y. Pinhasi, "Atmospheric and fog effects on ultra-wide band radar operating at extremely high frequencies," Sensors, vol. 16, no. 5, p. 751, 2016.

[48] W. Murphy and W. Hereman, "Determination of a position in three dimensions using trilateration and approximate distances," Department of Mathematical and Computer Sciences, Colorado School of Mines, Golden, Colorado, MCS-95, vol. 7, p. 19, 1995. 
[49] V. Nair and G. E. Hinton, "Rectified linear units improve restricted boltzmann machines," in Proceedings of the 27th international conference on machine learning (ICML10), 2010, pp. 807-814.

[50] F. Chollet, "keras," https://github.com/fchollet/keras, 2015.

[51] M. Abadi, A. Agarwal, P. Barham, E. Brevdo, Z. Chen, C. Citro, G. S. Corrado, A. Davis, J. Dean, M. Devin, S. Ghemawat, I. Goodfellow, A. Harp, G. Irving, M. Isard, Y. Jia, R. Jozefowicz, L. Kaiser, M. Kudlur, J. Levenberg, D. Mané, R. Monga, S. Moore, D. Murray, C. Olah, M. Schuster, J. Shlens, B. Steiner, I. Sutskever, K. Talwar, P. Tucker, V. Vanhoucke, V. Vasudevan, F. Viégas, O. Vinyals, P. Warden, M. Wattenberg, M. Wicke, Y. Yu, and X. Zheng, "TensorFlow: Large-scale machine learning on heterogeneous systems," 2015, software available from tensorflow.org. [Online]. Available: http://tensorflow.org/ 
Appendix A

2017 IEEE International Conference

on Multisensor Fusion and Integration for Intelligent Systems. 


\section{A Grid-based Indoor Radiolocation Technique Based on Spatially Coherent Path Loss Model}

\author{
Murat Ambarkutuk ${ }^{1}$
}

\author{
Tomonari Furukawa ${ }^{1}$
}

\begin{abstract}
This paper presents a grid-based indoor radiolocation technique based on a Spatially Coherent Path Loss Model (SCPL). Received Signal Strength (RSS) fingerprints are collected at different positions in the environment from which the radio wave propagation for the environment is empirically approximated with the SCPL model. Unlike the conventional path loss models, SCPL approximates radio wave propagation by first dividing the localization environment into grid cells and estimating the model parameters for each grid cell. Thus, the proposed technique is able to account for attenuation, resulting from non-uniform environmental irregularities. The efficacy of the proposed technique was investigated with an experiment comparing SCPL and an indoor radiolocation technique based on a conventional path loss model. The comparison has indicated the improved performance of the SCPL by up to $44 \%$.
\end{abstract}

\section{INTRODUCTION}

Radiolocation is a localization technique where some characteristics of radio waves are used to determine the position of the target of interest. Due to the existing ubiquitous availability of wireless networks and GPS-denied nature of indoor environments, radio waves have been regarded as a good candidate in indoor localization problems [1], [2]. While Received Signal Strength (RSS) is the most popular characteristics due to its setting with only one antenna, the bottleneck is the limited capability for information acquisition. The propagation of radio waves has been empirically modeled for a specific indoor environment accordingly [3], [4]. Of the empirical models, Log-distance Path Loss (LDPL) [5], which implicitly characterizes the attenuation caused by the obstructions in the environment, has been most popularly used for its strength in accuracy.

Recent improvement of accuracy of LDPL based radiolocation techniques can be attributed to two mainstream approaches: explicit attenuation modeling, and nondeterministic parameter estimation. The first explicit attenuation modeling is an approach where the accuracy is improved by refining the explicit formulation of attenuation. Bahl et al. [6] employed an LDPL variant in their radiolocation technique and explicitly modeled the attenuation caused by walls in the environment along with the implicit attenuation term. Their technique was employed with trilateration to globally determine the location of the agent. In their later work [7], the authors further modeled environment-caused attenuation, such as the number of people in the environment,

*This work was not supported by any organization

${ }^{1}$ Murat Ambarkutuk and ${ }^{1}$ Tomonari Furukawa are with the Mechanical Engineering Department, Virginia Polytechnic Institute and State University, Blacksburg, VA, 24060, USA, Email: \{murata, tomonari\}@vt.edu. by dynamically choosing different set of model parameters. Lim et al. [8] also focused on similar attenuation sources and employed Singular Value Decomposition (SVD) on the collected RSS to reduce the disturbances of the environmental attenuation on the fingerprints. Incorporation of explicit attenuation model into LDPL yielded highly accurate localization results for both techniques.

The nondeterministic parameter estimation approach, on the other hand, improves accuracy by refining parameters in the attenuation model. Chintalapudi et al. [9] introduced a Genetic Algorithm based approach for parameter estimation and applied it after localizing the access point positions as well as that of agent. Goswami et al. [10] proposed a learning-based approach for estimation of LDPL model parameters. The collected RSS fingerprints were represented with a Gaussian Mixture Model, and model parameters were estimated with the Expectation Maximization algorithm. Experimental results have validated the reduction of localization uncertainty caused by the proposed parameter estimation.

The aforementioned approaches significantly improved overall performance of LDPL based radiolocation techniques, but the radio wave propagation was approximated globally without considering the attenuation of a small region. In a typical warehouse, for instance, a fairly empty loading dock results in Line-of-sight (LOS) propagation path while the storage area, where racks are placed densely, shows Non-Line-of-sight (N-LOS) propagation characteristics. The warehouse, for instance, is typically piled with a large number of items and thus exhibits not only LOS but also N-LOS propagation characteristics. Employing a global propagation model without local attenuation would simply result in unwanted inaccurate localization.

Spatially Coherent Path Loss Model (SCPL) uniformly divides the environment into grid cells, the propagation parameters are then estimated for each grid cell. Therefore, both the path loss and the attenuations can be characterized locally. At an unknown location, the agent receives a set RSS fingerprints collected from various access points from which the distance between access points and the agent is determined by employing the approximated propagation parameters. The range measurements are then used to attain the global position of the agent. The grid cell representation of the environment is then further incorporated into localization by representing the results as probability distribution over the grid cells.

The organization of the paper is as follows. Section II lays out the fundamentals of the radiolocation by introducing trilateration and LDPL model. Section III presents the details 
of the proposed technique based on the SCPL. Section IV investigates the validity of the proposed technique. Section V concludes this paper and discusses ongoing future work.

\section{Radio WAVE PROPAGATION IN INDOOR RADIOLOCATION}

In this section, trilateration, a common pose-fixing technique for indoor radiolocation problems, is first introduced. The formulation of LDPL model, with which RSS fingerprints can be used for indoor localization purposes, is then given.

\section{A. Indoor Radiolocation with Trilateration}

The indoor radiolocation problem of interest in this paper is to locate an agent equipped with a radio module in an indoor environment that installs some number of access points in a distributed manner. Let indoor environment and some number of access points with known poses in the environment be $\Omega$ and $m_{1: k}$, respectively. At an unknown location, the agent collects $n_{m}$ number RSS fingerprints from all the access points within its field of coverage. The distances between the agent and the access points can be inferred with the use of RSS fingerprints and the radio wave propagation model. Consequently, the global position of the agent can be then obtained by using the trilateration method.

Let the measured distance between the agent and $i^{\text {th }}$ access point which resides at $\vec{x}^{i}=\left[x^{i}, y^{i}, z^{i}\right]^{T}$ be $d_{i}$. Then the position of the agent can be represented as:

$$
d_{i}^{2}=\left(x^{t}-x^{i}\right)^{2}+\left(y^{t}-y^{i}\right)^{2}+\left(z^{t}-z^{i}\right)^{2}
$$

As can be seen in the Equation (1), each equation induces a sphere centered at the access point with $d_{i}$ radius. Since all the access points in the field of coverage of the agent lead to an equation, simultaneous solution of these equation yields to the position of the agent.

By using $j^{t h}$ equation as a linearization pivot, all the quadratic unknown terms can be eliminated in Equation (1). Therefore, the set of equations attained can be represented in a matrix form. The linear system can be then solved for

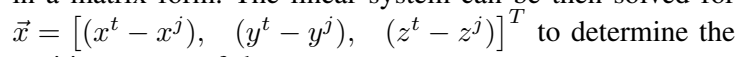
position vector of the agent.

$$
\boldsymbol{A}=\left[\begin{array}{ccc}
x^{1}-x^{j} & y^{1}-y^{j} & z^{1}-z^{j} \\
x^{2}-x^{j} & y^{2}-y^{j} & z^{2}-z^{j} \\
\vdots & \vdots & \vdots \\
x^{j-1}-x^{j} & y^{j-1}-y^{j} & z^{j-1}-z^{j} \\
x^{j+1}-x^{j} & y^{j+1}-y^{j} & z^{j-1}-z^{j} \\
\vdots & \vdots & \vdots \\
x^{n_{m}}-x^{j} & y^{n_{m}}-y^{j} & z^{n_{m}}-z^{j}
\end{array}\right]
$$

$$
\vec{b}=0.5\left[\begin{array}{c}
d_{j}^{2}-d_{1}^{2}+d_{1 j}^{2} \\
d_{j}^{2}-d_{2}^{2}+d_{2 j}^{2} \\
\vdots \\
d_{j}^{2}-d_{j-1}^{2}+d_{j-1 j}^{2} \\
d_{j+1}^{2}-d_{j+1}^{2}+d_{j+1 j}^{2} \\
\vdots \\
d_{j}^{2}-d_{n_{m}}^{2}+d_{n_{m}, j}^{2}
\end{array}\right]
$$

where $d_{i j}$ represents the distance between $i^{\text {th }}$ and $j^{\text {th }}$ markers. Due to sensor and process uncertainty, direct solution of trilateration often yields to unwanted solutions [11]. Therefore, the least squares approach is often used in trilateration problems to cope with noisy measurements. The least square formulation minimizes overall localization error $S$, which is induced by the measurement noise.

$$
\begin{aligned}
& S=\vec{r}^{T} \vec{r}=(\vec{b}-\boldsymbol{A} \vec{x})^{T}(\vec{b}-\boldsymbol{A} \vec{x}) \\
& \overrightarrow{\hat{x}}=\underset{x}{\arg \min } S
\end{aligned}
$$

If the $\boldsymbol{A}^{T} \boldsymbol{A}$ is not singular, the solution for Equation (2) can be derived as Equation (3).

$$
\overrightarrow{\hat{x}}=\left(\boldsymbol{A}^{T} \boldsymbol{A}\right)^{-1} \boldsymbol{A}^{T} \vec{b}
$$

\section{B. Log-distance Path Loss Model}

LDPL is an indoor wave propagation model which formulates the correlation between the transmitted and received signal strength as a function of Euclidean distance between an access point and the agent.

Let $P L(d)$ be the difference between transmitted signal strength and RSS fingerprint obtained at a test point $d$ distance away from the access point. This difference is commonly referred as path loss. LDPL model, formulates the path loss of the test point relative to a reference point $d_{0}$ distance away from the access point:

$$
P L(d)=\overline{P L}\left(d_{0}\right)+10 n \log _{10} \frac{d}{d_{0}}+X_{\sigma}
$$

where $\overline{P L}\left(d_{0}\right)$ and $X_{\sigma}$ are the mean path loss measured at the reference point, a normal distribution with zero mean and $\sigma$ standard deviation, respectively. The second term in the model denotes the relative path loss as a function of $\frac{d}{d_{0}}$ and path loss exponent $n$. Table I shows different values of path loss exponent $n$ depending on the environment and communication path.

As can be seen in the formulation of the LDPL model, wave propagation not only depends on separation distance $d$, but also path loss exponent $n$ and standard deviation $\sigma$. Therefore, these parameters should be estimated from the collected data. A least squares approach can be used to obtain the model parameters:

$$
n^{*}, \sigma^{*}=\underset{n, \sigma}{\arg \min } \frac{1}{n_{s}} \sum_{i=1}^{n_{s}}\|P L(d)-\widehat{P L}(d)\|_{2}^{2}
$$


where $\widehat{P L}(d)$ is the estimated path loss occurred at the distance $\mathrm{d}$ with path loss $n$ and standard deviation $\sigma$.

LDPL formulation can be solved for the Euclidean distance $d$, for each measured path loss $P L(d)$ at an unknown location, and estimated model parameters $\left[n^{*}, \sigma^{*}\right]^{T}$. Consequently, each fingerprint collected from access points within the agent's radio coverage will induce a circle in $\mathbb{R}^{2}$ centered at the access point. The agent location can be then determined with trilateration applied on the induced circles.

TABLE I: Various values of path loss exponent based on the environment and the propagation path.

\begin{tabular}{ll}
\hline$<2$ & $\begin{array}{l}\text { the space structure guides the radio waves along the } \\
\text { propagation path }\end{array}$ \\
\hline$=2$ & $\begin{array}{l}\text { empty space overall propagation path is dominated } \\
\text { LOS communication }\end{array}$ \\
\hline$>2$ & $\begin{array}{l}\text { There are obstructions along the propagation path } \\
\text { multipath effect takes places due to N-LOS commu- } \\
\text { nication }\end{array}$ \\
\hline
\end{tabular}

\section{GRID BASED Indoor Radiolocation TechniQue} Based on Spatially Coherent Path Loss

Figure 1 demonstrates the schematic diagram of the proposed grid-based indoor radiolocation technique based on SCPL. Given an RSS fingerprint obtained at an unknown location, the model parameters are first chosen with a feedforward neural network. The localization function based on SCPL is then used to estimate to the distances between the agent, and the access points. Least-squares trilateration technique is successively applied on the attained distances to determine the location of the agent. Consequently, the result of the trilateration is represented with a probabilistic distribution over the indoor localization environment $\Omega$. This section covers the details of each component of the localization algorithm in further detail.

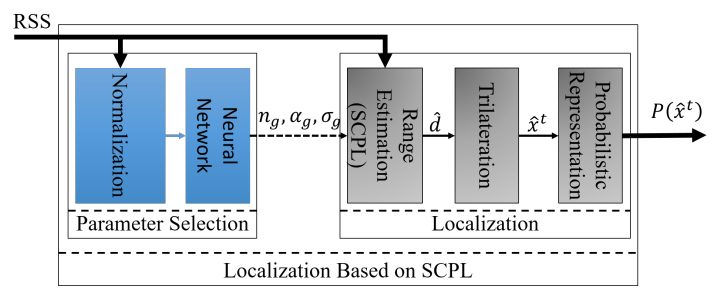

Fig. 1: Proposed localization algorithm: The model parameters are first chosen with a feed-forward neural network. The distance between the agent and the access points is attained with SCPL. Consequently, trilateration is used to determine the position of the agent which results in a belief function over the localization environment $\Omega$.

\section{A. Spatially Coherent Path Loss Model}

Let $\Omega=\left\{g(i, j) \mid i=1 \ldots n_{g x}, j=1 \ldots n_{g y}\right\}$ be the localization environment which is uniformly divided $n_{g x}$ by
TABLE II: The descriptions of the SCPL model parameters.

\begin{tabular}{ll}
\hline$n_{g}$ & $\begin{array}{l}\text { The path loss exponent estimated for grid cell } g . \text { If the grid } \\
\text { cell } g \text { is closer to an obstruction, it is expected to see higher } \\
\text { values in order to model the attenuation better. }\end{array}$ \\
\hline$\alpha_{g} \quad \begin{array}{l}\text { Distance correction factor. Since the N-LOS propagation path } \\
\text { should be longer than LOS path, the length of the propagation } \\
\text { path is corrected with this parameter. }\end{array}$ \\
\hline$\sigma_{g} \quad \begin{array}{l}\text { The estimated deviation of the normal distribution which is } \\
\text { modeling the slow fading of the channel. }\end{array}$ \\
\hline
\end{tabular}

$n_{g y}$ number of grid cells. $g(i, j)$ represents the grid cell resides in $[i, j]$ in grid-coordinates. Figure 2 demonstrates the grid cell structure of the environment in detail. SCPL model characterizes the radio wave propagation for each grid cell by evaluating the formulation below:

$$
P L(d)=P L_{l o s}(d)+P L_{a t t}(d)+X_{\sigma}
$$

SCPL model characterizes the measured path loss acquired at a test point with $d$ distance away from the access point as the sum of the LOS propagation path loss $P L_{l o s}(d)$ and an explicit attenuation term $P L_{a t t}(d) . P L_{l o s}(d)$ is defined with Friis' Free Space Equation (FFSE) [12]. On the other hand, $P L_{a t t}(d)$ the attenuation caused by the obstacles in grid cell $g$ is formulated as given in Equation (7).

$$
P L_{a t t}(d)=10 n_{g} \log _{10} \alpha_{g} d
$$

where $n_{g}$ and $\alpha_{g}$ represent the path loss exponent and distance correction factor for grid cell $g$. Since the N-LOS propagation path will be longer than LOS path, $\alpha_{g}$ estimates the N-LOS distance, while $n_{g}$ serves as a path loss exponent. Table II denotes the model parameters and their descriptions.

Similar to attenuation term in LDPL, SCPL models the attenuation as a logarithmic function. However, the main difference is that SCPL does not model the attenuation relative to the reference point. Rather, the path loss exponent $n_{g}$, the distance correction factor $\alpha_{g}$ and $X_{\sigma_{g}}$ is a function of the position the agent. For each grid cell, model parameters are estimated; hence, the propagation of the radio waves are identified locally. As a result of this, for each grid cell, the irregularities of the environment can be modeled locally.

SCPL model parameters are estimated with a least-squares approach which minimize the path loss estimation error in grid cell $g$. Once these parameters are estimated from the collected fingerprints, the proposed path loss model can be used to estimate the separation distance from all of the access points. The formulation for the estimation is given in Equation (8).

$$
\begin{aligned}
& n^{*}(i, j), \alpha^{*}(i, j), \sigma^{*}(i, j)= \\
& \underset{n, \alpha, \sigma}{\arg \min } \frac{1}{n_{s} n_{x} n_{y}} \sum_{v=j-\frac{n_{y}}{2}}^{j+\frac{n_{y}}{2}} \sum_{u=i-\frac{n_{x}}{2}}^{i+\frac{n_{x}}{2}} \sum_{s=1}^{n_{s}}\|P L(u, v)-\widehat{P L}(i, j)\|_{2}^{2}
\end{aligned}
$$


where $P L(u, v)$ represents the path loss measured in grid cell $g(u, v)$, while $n_{s}$ represent the number of fingerprints used in the estimation.

As can be seen in Equation (8), for each grid cell $g(i, j)$, the loss function considers neighboring grid cells with spread factor of $n_{x}$ and $n_{y}$. Assuming grid cells residing in a local vicinity should show similar propagation characteristics, the parameters of grid cell $g(i, j)$ are attained by employing the fingerprints collected in neighboring grid cells as well. By incorporating the neighboring grid cells, the data points needed to estimate the model parameters are decreased, as well as the locality coherence is ensured.

\section{B. Grid-based Radiolocation Based on SCPL Model}

Figure 1 demonstrates the schematic diagram of the proposed localization technique. Given a fingerprint $P_{r}(i, j)$ acquired in an unknown grid cell $g(i, j)$, the neural network is first employed to choose a set of SCPL model parameters. After selecting a set of model parameters, the acquired fingerprint $P_{r}(i, j)$ is used to estimate the distances between the access points and the agent. Since SCPL model results in a distribution for each distance estimation, the trilateration also yields a distribution representing the position of the agent.

1) Parameter Selection: Given a fingerprint $P_{r}(i, j)$ acquired in an unknown grid cell $g(i, j)$, the neural network is first employed to choose a set of SCPL model parameters. Let $\vec{p}(i, j)=[n(i, j), \alpha(i, j), \sigma(i, j)]^{T} \in \mathbb{R}^{3}$ are first chosen from all the estimated parameters $\boldsymbol{P} \in \mathbb{R}^{n_{g x} \times n_{g_{y}} \times 3}$. This parameter selection is achieved with a 3-layer feed-forward neural network where each layer of the network can be represented as Equation (9).

$$
f_{n}\left(\vec{h}_{n}\right)=\sigma\left(\boldsymbol{W}_{n} \vec{h}_{n}+\vec{b}_{n}\right)
$$

where $\boldsymbol{W}_{n}$ and $\vec{b}_{n}$ represent the learned weight matrix and bias vector of $n^{\text {th }}$ layer, respectively. $\sigma(\cdot)$ denotes the nonlinearity function, while $\vec{h}_{n}$ is the input of the $n^{\text {th }}$ hidden layer. Since all the model parameters are positives values, ReLu [13] is used as the nonlinearity function in SCPL model. Before training the neural network the input data, the measurements, is normalized such that the measurements of the each access points centered at zero with a variance of 1 .

Consequently, the model parameters are inferred by utilizing weights and biases of each layer consecutively, followed by the nonlinearity function.

2) Localization: After model parameters are chosen, the distance from the access point and to the agent is be estimated by utilizing the SCPL model. Trilateration is then be employed successively in order to determine the location of the agent. Finally, the result of the trilateration algorithm is transformed to probabilistic representation.

Let $P(i, j)$ be the probability of the agent residing in $g(i, j)$, resulted from the least-squares trilateration. Then $\mathbb{P}(\Omega)$ represents the probability distribution function over all the grid cells.
The quantification of the probability distribution function $\mathbb{P}(\Omega)$ is then achieved with expectation operator. The final localization result is represented as below:

$$
\overrightarrow{\hat{x}}^{t}=\mathbb{E}[\mathbb{P}(\Omega)]
$$

By representing the localization as a distribution over $\Omega$, the model can better account for error resulted from model inaccuracies, environment irregularities and trilateration.

\section{EXPERIMENTATION}

This section will outline the details of the experimentation conducted. The structure of the environment is first described as well the determination of the grid cell size, followed by the details of the fingerprint collection process. Finally, evaluation of the parameter estimation, parameter selection and the overall SCPL model performance is covered.

\section{A. Structure of the Environment and Grid cell Size Determi- nation}

The experimentation was conducted in Hancock Hall, Virginia Tech, where the environment can be considered empty. The environment contains two support columns inside and it is closed from three edges with cinder block walls while the last edge was open to another open area. The environment is 22.5 by 7.2 meters, in which 8 access points running at $900 \mathrm{MHz}$ were placed. Figure 2 displays the grid cell representation of the environment, and the location of the access points. The characteristics of the radio modules

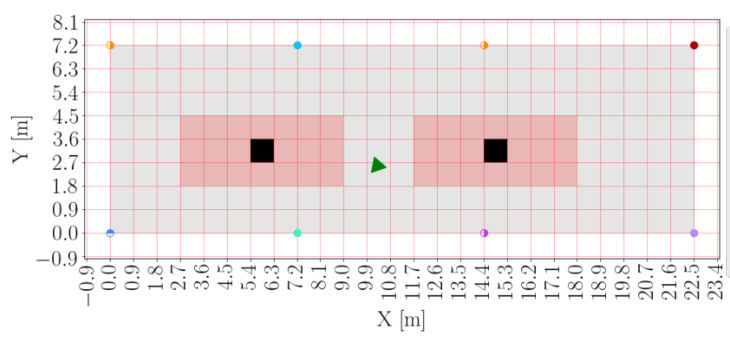

Fig. 2: Grey grid cells demonstrate the localization environment $\Omega$. Grey triangle is the pose of the agent. The circles represent the access points while black regions depict the support columns. The grid cells marked with red color represent the regions where the fingerprints corresponding to the grid cells in shadowing effect are contributed into parameter estimation.

used plays a significant role in the the determination of the grid cell size. Fraunhofer distance was considered to determine the size of the grid cells such that the fingerprints were acquired within far-field propagation zone $(d>2 \lambda)$. The grid cell size determined as 0.9 by 0.9 meters as the radios, used in the experimentations, were running at 900 $\operatorname{MHz}(\lambda=0.33$ meters $)$, 


\section{B. Parameter Estimation for SCPL Model}

In Figure 2, grid cells marked with red color represent the regions where obstructions affected during the parameter estimation. Therefore, around that region, SCPL parameters should show an increase in order to capture the increased attenuation. Figure 3 shows the path loss exponent parameter of the SCPL model. Around the obstructions, path loss exponent shows increase.

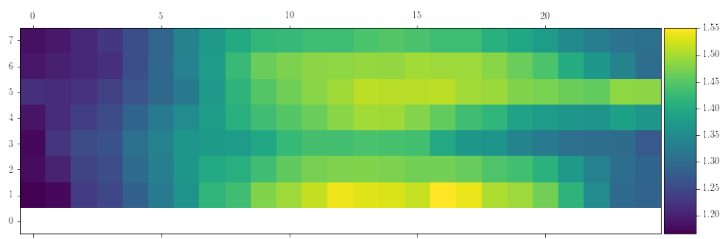

Fig. 3: Estimated path loss parameters for each grid cells. As it can be seen, the grid cells neighboring obstructions tend to have larger path loss exponent, which shows attenuation in these vicinities have more impact on the localization.

As described in Section III, a three layer feed-forward neural network was employed with which the parameters of model $\left(n_{g}, \alpha_{g}, \sigma_{g}\right)$ are chosen. The network is trained with Stochastic Gradient Descent algorithm with Nesterov momentum updates.

\section{Analysis of Range Estimations}

In order to analyze the efficacy of the SCPL model, first the range estimation is analyzed. For each fingerprints collected at an unknown position, SCPL and LDPL are separately used to estimate the range from all access points residing in the observable region. The error metric for each fingerprint vector is given below.

$$
e_{1 d}=\frac{1}{n_{s} n_{a n}} \sum_{i=1}^{n_{s}} \sum_{j=1}^{n_{a n}}\left\|\hat{d}_{i}^{j}-d_{i}^{j}\right\|
$$

Table III tabulates the estimated separation distance error for LDPL, SCPL models. SCPL is able to localize the agent with 5.2 meters mean localization performance, while LDPL achieves 14.98 meters.

The last row of the Table III denotes the 95\% level confidence intervals for each algorithm. The true mean of error distribution of LDPL and SCPL in range estimation is between 7.91 and 22.05 meters, and between 4.98, and 5.43 meters, respectively.

Figure 4 depicts the empirical cumulative distribution function of the error in range estimations. At $80 \%$ frequency level, the performance of the SCPL degrades from around 7 meters (red line in Figure 4) to around 8 meters error (brown line), by using N-LOS fingerprints. On the other hand, at time same frequency level LDPL degrades from 11 meters to 16 meters.

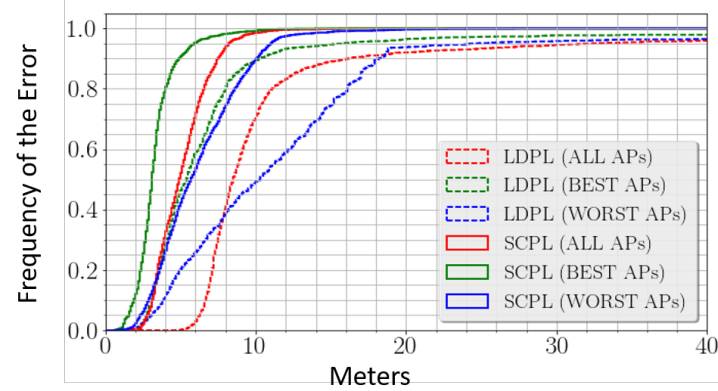

Fig. 4: Cumulative distribution function of range estimation errors

TABLE III: Comparison of Errors in Range Estimation

\begin{tabular}{lccc}
\hline Error Metric & LDPL & SCPL (w/o NN) & SCPL (w/ NN) \\
\hline Mean Error $[\mathrm{m}]$ & 14.98 & $\mathbf{5 . 1 9}$ & 5.20 \\
\hline Median Error $[\mathrm{m}]$ & 7.17 & $\mathbf{4 . 1}$ & 4.11 \\
\hline Std. Dev. $[\mathrm{m}]$ & 141.79 & 4.68 & $\mathbf{4 . 6 1}$ \\
\hline Confidence Interval & {$[7.91,22.05]$} & {$[\mathbf{4 . 9 7}, \mathbf{5 . 4 3}]$} & {$[4.98,5.43]$} \\
\hline
\end{tabular}

\section{Analysis of Target Localization}

The performance of SCPL model was also analyzed after trilateration technique is employed. The error metric used in the analysis is given in Equation (12).

$$
e_{2 d}=\frac{1}{n_{s} n_{a n}} \sum_{i=1}^{n_{s}} \sum_{j=1}^{n_{a n}}\left\|\hat{x}_{i}^{j}-x_{i}^{j}\right\|_{2}
$$

As shown in Table IV, after trilateration, the performance of

TABLE IV: Comparison of Errors in Target Localization

\begin{tabular}{l|l|ll}
\hline & LDPL & \multicolumn{2}{|c}{ SCPL } \\
\hline Error Metric & Tri & Tri* & Tri* + GL \\
\hline Mean Error $[\mathrm{m}]$ & 5932.56 & 10.05 & $\mathbf{7 . 2 7}$ \\
\hline Median Error $[\mathrm{m}]$ & 8.66 & 8.11 & $\mathbf{7 . 1}$ \\
\hline Std. Dev. [m] & 124988 & 12.60 & $\mathbf{2 . 7 2}$ \\
\hline Confidence Interval & {$[-303.82,12168]$} & {$[9.46,10.99]$} & {$[\mathbf{7 . 1 4}, \mathbf{7 . 4 1}]$} \\
\hline
\end{tabular}

both LDPL and SCPL degraded. By using a neural network to choose model parameters, SCPL achieved 7.1 median localization in target location estimation. On the other hand, LDPL was able to localize the agent with 8.66 median localization error.

Figure 5 depicts the empirical cumulative distribution function of the error in target localization. As can be seen in the figure, when the collected fingerprints consist of only LOS measurements, then SCPL and LDPL follow the same trend. At $80 \%$ frequency level, the performance of the SCPL degrades from around 10 meters to around 12 meters error, by using N-LOS fingerprints. On the other hand, at time 


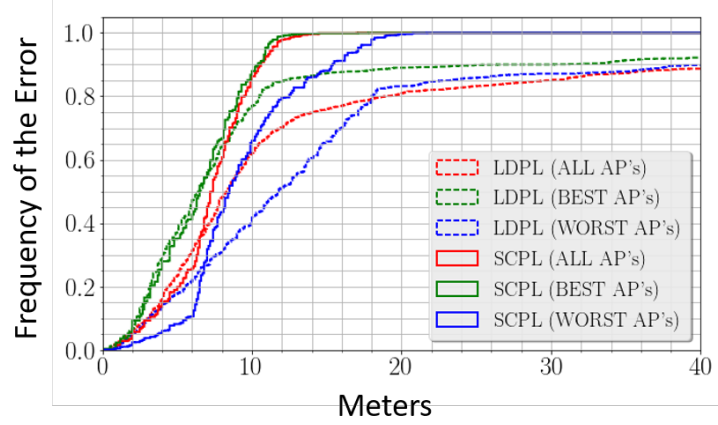

Fig. 5: Cumulative distribution function of target localization error.

same frequency level LDPL degrades from 10 meters to 18 meters.

Figure 6 depicts the error distribution in target localization over the grid cells. The right most column in the fig. 6 depicts that LDPL and SCPL shows similar localization results if the best access points are used. Localization performance of LDPL degrades significantly more than SCPL if NLOS fingerprints (Figure 6, center column) were used for localization. These analyses suggests that explicit attenuation modeling limits the effect of obstructions on the path loss model and radiolocation technique, ensuring better localization results.

\section{E. Access Point Analysis}

In order to assess effect of the individual access points location in the target localization, the error contribution of each access point in the target localization is also analyzed. Table V tabulates the mean error contribution of each access point across the dataset. The table clearly shows that the distribution of error contribution of each access point is not uniform.

\begin{tabular}{lcccccccc}
\hline & AP0 & AP1 & AP2 & AP3 & AP4 & AP5 & AP6 & AP7 \\
\hline LDPL & 33.33 & 7.86 & 6.77 & 19.14 & 13.76 & 18.1 & 9.6 & 11.28 \\
\hline SCPL & 6.63 & 3.52 & 3.13 & 6.26 & 6.99 & 5.34 & 3.57 & 6.15 \\
\hline
\end{tabular}

TABLE V: Error Statistics of Access Points in Separation Distance Estimation

In order to further quantify the effect, the fingerprints of the best 3 access points employed to determine to location of the agent. All three combinations of all (8) access points measurements are employed to estimate the separation distance while the remaining fingerprints were discarded. With the combinatorial analysis, access points denoted with 1,2 , 6 (see Figure 2) found to be the least contributor in the error, while the combination of $3,4,5$ seem to perfom the worst in our dataset. By only using the access points found to be more reliable, the error deviation of LDPL model reduced significantly, while the mean error of LDPL and SCPL models decreased by $46 \%$ and $34 \%$, respectively. On the other hand, worst-3 analysis demonstrates that the mean error of LDPL and SCPL models increased $13 \%$ and 20\%, respectively. Detailed error statistics of best-3 and worst-3 analysis tabulated in Table VI.

\begin{tabular}{lccc}
\hline \multicolumn{4}{c}{ Best-3 Access Points } \\
\hline Error Metric & LDPL & SCPL (w/o NN) & SCPL (w/ NN) \\
\hline Mean Error [m] & 8.07 & $\mathbf{3 . 2 8}$ & 3.39 \\
\hline Median Error [m] & 5.03 & $\mathbf{2 . 8 4}$ & 2.87 \\
\hline Std. Dev. [m] & 27.84 & $\mathbf{2 . 7 9}$ & 2.82 \\
\hline Confidence Interval & {$[6.69,9.47]$} & {$[\mathbf{3 . 1 4}, \mathbf{3 . 4 2}]$} & {$[3.25,3.53]$} \\
\hline \multicolumn{4}{c}{ Worst-3 Access Points } \\
\hline Error Metric & LDPL & SCPL (w/o NN) & SCPL (w/ NN) \\
\hline Mean Error [m] & 16.99 & $\mathbf{6 . 0 3}$ & 6.25 \\
\hline Median Error [m] & 9.19 & $\mathbf{5 . 0 1}$ & 5.1 \\
\hline Std. Dev. [m] & 108.189 & $\mathbf{5 . 1 9}$ & 5.21 \\
\hline Confidence Interval & {$[11.60,22.39]$} & {$[\mathbf{5 . 7 7 , 6 . 3 0}]$} & {$[5.99,6.51]$} \\
\hline
\end{tabular}

TABLE VI: Comparison of Error Statistics in Separation Distance Estimation with Best 3 and Worst 3 access points

\section{CONClusions AND Future Work}

This paper has presented a grid-based indoor radiolocation technique based on the SCPL. The wave propagation is estimated for each grid cell so that the obstructions can only effect the propagation model in a local vicinity. The grid cell representation of the environment is furthered incorporated it into localization by representing the results as probability distribution over the grid cells.

This ability enabled SCPL to capture the attenuation caused by the environment, and to significantly improve (up to $44 \%$ ) the localization performance in range estimations in comparison to LDPL. In the light of the experimentations, SCPL display more robustness to error caused by the N-LOS fingerprints than LDPL. Overall, the proposed grid-based indoor radiolocation technique yielded up to $18 \%$ (median) target localization improvement over LDPL based radiolocation technique. Results have demonstrated the applicability of the proposed approach in unstructured environments.

This paper has focused on a grid-based indoor radiolocation technique based on SCPL which can account for obstruction dissimilarity of the environment. However, much work is still left open. Not every access point results in the same extent of error; further investigation must be done to quantify the error contribution of each access point from which an active access point selection can be employed to further reduce the localization error.

\section{REFERENCES}

[1] S. He and S.-H. G. Chan, "Wi-fi fingerprint-based indoor positioning: Recent advances and comparisons," IEEE Communications Surveys \& Tutorials, vol. 18, no. 1, pp. 466-490, 2016. 


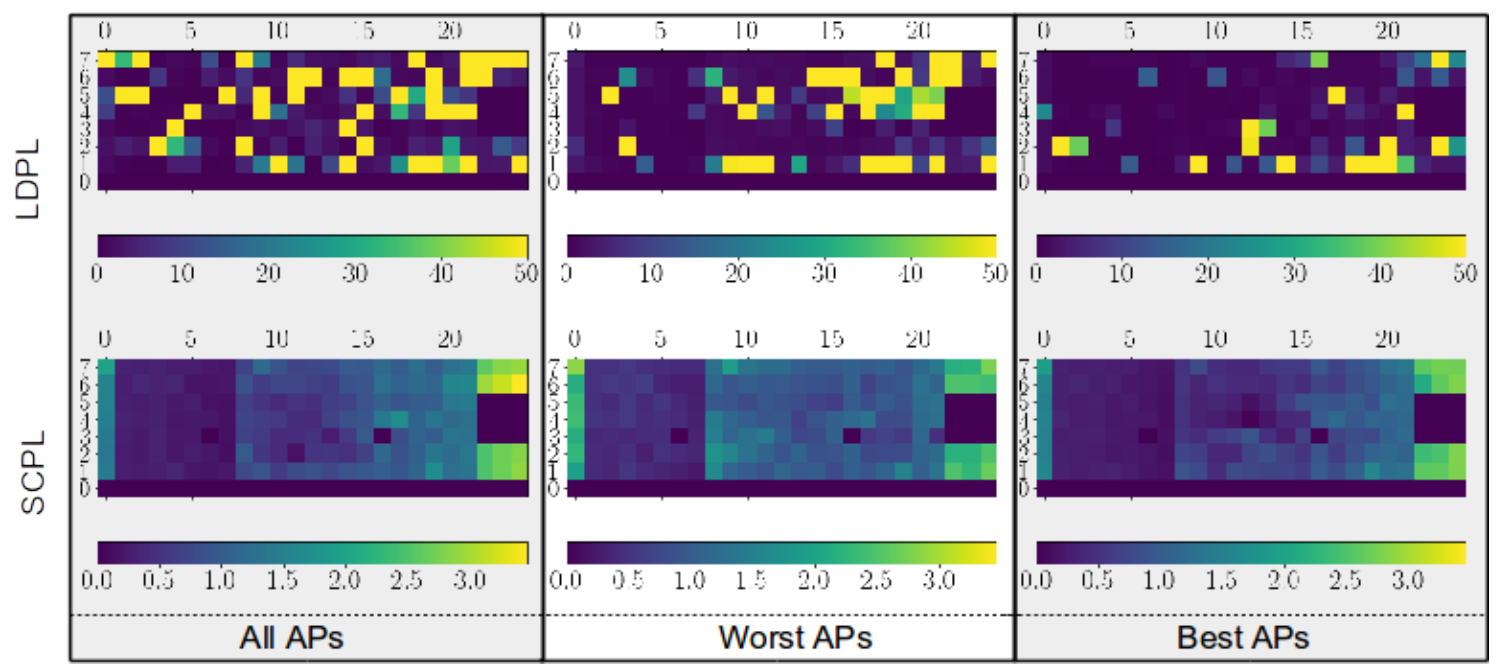

Fig. 6: Error distribution of both algorithms over grid cells.

[2] A. M. Hossain and W.-S. Soh, "A survey of calibration-free indoor positioning systems," Computer Communications, vol. 66, pp. 1-13, 2015.

[3] J. S. Seybold, Introduction to RF propagation. John Wiley \& Sons, 2005.

[4] J. B. Andersen, T. S. Rappaport, and S. Yoshida, "Propagation measurements and models for wireless communications channels," IEEE Communications Magazine, vol. 33, no. 1, pp. 42-49, 1995.

[5] T. S. Rappaport et al., Wireless communications: principles and practice. prentice hall PTR New Jersey, 1996, vol. 2.

[6] V. Bahl and V. Padmanabhan, "Radar: An in-building rfbased user location and tracking system." Institute of Electrical and Electronics Engineers, Inc., March 2000. [Online]. Available: https://www.microsoft.com/en-us/research/publication/ radar-an-in-building-rf-based-user-location-and-tracking-system/

[7] P. Bahl, V. N. Padmanabhan, and A. Balachandran, "Enhancements to the radar user location and tracking system," Microsoft Research, vol. 2, no. MSR-TR-2000-12, pp. 775-784, 2000

[8] H. Lim, L.-C. Kung, J. C. Hou, and H. Luo, "Zero-configuration, robust indoor localization: Theory and experimentation," Tech. Rep., 2005.

[9] K. Chintalapudi, A. Padmanabha Iyer, and V. N. Padmanabhan, "Indoor localization without the pain," in Proceedings of the sixteenth annual international conference on Mobile computing and networking. ACM, 2010, pp. 173-184.

[10] A. Goswami, L. E. Ortiz, and S. R. Das, "Wigem: A learningbased approach for indoor localization," in Proceedings of the Seventh COnference on emerging Networking EXperiments and Technologies. ACM, 2011, p. 3.

[11] W. Murphy and W. Hereman, "Determination of a position in three dimensions using trilateration and approximate distances," Department of Mathematical and Computer Sciences, Colorado School of Mines, Golden, Colorado, MCS-95, vol. 7, p. 19, 1995.

[12] H. T. Friis, "A note on a simple transmission formula," Proceedings of the IRE, vol. 34, no. 5, pp. 254-256, 1946.

[13] V. Nair and G. E. Hinton, "Rectified linear units improve restricted boltzmann machines," in Proceedings of the 27th international conference on machine learning (ICML-10), 2010, pp. 807-814 